\title{
IV. AUF DEM WEG ZUM VERFASSUNGSSTAAT? VERFASSUNGSPOLITIK UND KONSTITUTIONELLE ENTWICKLUNG
}

Der Umbau des Großherzogtums Berg zu einem einheitlichen und effizienten Staatswesen erschöpfte sich nicht in der Modernisierung der hergebrachten Verwaltung, sondern erstreckte sich ebenfalls auf die Neugestaltung der Verfassungsstrukturen. Beide Aspekte standen in enger Korrelation zueinander, denn die Reformen im administrativen Bereich leisteten einen wichtigen Beitrag zur konstitutionellen Neuordnung des Großherzogtums, mehr noch: Die Administration war der Baustein der Verfassung.

Es war Joachim Murat, der unmittelbar nach seinem Regierungsantritt erste Schritte einleitete, um die Verfassungsstrukturen der ihm übertragenen Länder neu zu gestalten. Seine Bemühungen richteten sich hierbei zunächst auf den Erlaß einer kodifizierten Verfassung. Es stellte sich jedoch heraus, daß Napoleon die Konstitutionalisierung des Landes nicht guthieß. Der Prinz nahm deshalb Abstand von seinen Plänen, den Staat im Rahmen eines Verfassungsaktes $\mathrm{zu}$ reorganisieren, und förderte statt dessen die konstitutionelle Entwicklung des Landes mit Hilfe von Einzelgesetzen. Diese ließen sich in Paris leichter durchsetzen als der Erlaß einer Konstitution. Die evolutionäre Umgestaltung ersetzte somit den revolutionären Reformansatz. Einzelne Punkte, wie die Etablierung von Repräsentativorganen, blieben zwar zunächst noch an die Konstitutionalisierung gebunden. Da sie als Verfassungsfragen im engeren Sinne betrachtet wurden, galt der Erlaß einer kodifizierten Verfassung für ihre Durchsetzung anfangs als Conditio sine qua non. Doch mit dem Ausbleiben einer Konstitutionsakte wurden auch diese Fragen von der Gewährung einer Verfassung gelöst und separat bewältigt.

\section{Verfassungsdiskussionen}

Der Erlaß einer geschriebenen Verfassung gehörte zu jenen Sachverhalten, die bis zum Zusammenbruch des Großherzogtums nicht definitiv geklärt wurden, aber auch nicht vollständig von der politischen Tagesordnung verschwanden. Auffällig ist hierbei, daß sich die Regierung immer dann mit der Verfassungsfrage beschäftigte, wenn staatspolitische Veränderungen eingetreten waren oder zur Debatte standen. Um den Erlaß einer kodifizierten Verfassung ging es erstmals, nachdem Prinz Murat die Herrschaft in Düsseldorf übernommen hatte (1). Nach den Gebietserweiterungen im Januar 1808 und nach dem Übergang der Großherzogswürde an Napoleons Neffen Louis Napoléon im März 1809 kam das Thema abermals zur Sprache (2). Zuletzt war die Verfassungsfrage im Gespräch, als im Herbst 1809 über Veränderungen in der Regierungsorganisation nachgedacht wurde (3). 
(1) Nur wenige Wochen nach Übernahme der Regierung ließ Murat den französischen Kaiser wissen, daß er beabsichtige, für Kleve und Berg eine gemeinsame Verfassung zu erlassen. Nicht die französischen Verfassungsverhältnisse, sondern die Strukturen vor Ort sollten Fundament der geplanten Konstitution werden. Je vais charger deux ou trois personnes, teilte er Napoleon mit, versées dans la connaissance des lois et des intérêts des deux pays, de rédiger un projet de Constitution qui leur convienne ${ }^{1}$. Der Innovationsbereitschaft des neuen Landesherrn lagen primär machtpolitische Überlegungen zugrunde. Murat bezweckte mit der zukünftigen Verfassung, seiner gerade erst erworbenen Herrschaft Rückhalt und Stabilität zu verleihen. Nach außen zielte er darauf, die noch unsichere politische Existenz des neuen Staates zu festigen und diesen aus der Abhängigkeit von der Pariser Zentrale zu lösen. Nach innen wollte er gesamtstaatliche Identität erzeugen und notwendige Grundlagen für die Durchsetzung der Reformziele in Regierung und Verwaltung schaffen - offensichtlich kannte der Prinz sehr genau die Funktion von Verfassungen und war sich über deren gewachsene Bedeutung im klaren. Damit ist wohl auch zu begründen, daß Murat bei der Realisierung seiner Verfassungspläne keine Zeit verlor. Nur fünf Tage, nachdem er den Kaiser von seinen Plänen in Kenntnis gesetzt hatte, schickte er einen ersten Konstitutionsentwurf zur Begutachtung nach Paris ${ }^{2}$. Der Entwurf stammte aus der Feder seines Sekretärs in Düsseldorf, des Grafen Agar. Einheimische Kräfte waren an der Ausarbeitung des Projekts nicht beteiligt worden, obwohl der Landesherr dies zunächst angekündigt hatte.

Es ist bereits darauf hingewiesen worden, daß der Konstitutionsentwurf in der französischen Hauptstadt keinen Beifall fand und deshalb auch nicht realisiert wurde. Napoleon bezeichnete das Projekt als überstürzt und verfrüht - ein Vorwurf, den er angesichts der kurzen Zeitspanne, in der das Dokument ausgearbeitet worden war, sicherlich nicht ganz zu Unrecht erhob. Ebenso verwarf er die inhaltliche Ausgestaltung des Verfassungsentwurfs. Insbesondere kritisierte er die starke Rücksichtnahme auf lokale Besonderheiten. Seiner Meinung nach durfte sich die zukünftige Verfassung der Herzogtümer nicht an den einheimischen Verhältnissen, sondern mußte sich vielmehr an den Maximen der französischen Verfassung orientieren ${ }^{3}$. Im Gegensatz zu Murat sah Napoleon in der Konstitutionsakte nämlich hauptsächlich ein Mittel zur Durchsetzung seiner außenpolitischen Ziele. Ihm ging es darum, in der deutschen Staatenwelt Werbung für die französische Herrschaft zu machen. Daß er den Verfassungsentwurf ablehnte, hing aber wohl auch damit zusammen, daß er die beiden Herzogtümer zu diesem Zeitpunkt

1 Joachim Murat in seinem Schreiben an Napoleon vom 28. März 1806, abgedruckt in: Lettres et documents, Bd.4, Nr.2289, S.184f., Zitat S. 187.

2 Das geschah am 31.März 1806, wie aus dem bereits mehrfach zitierten Schreiben Murats an Napoleon vom 31. März 1806, abgedruckt in: ibid. Nr.2293, S.190f., hervorgeht. Vgl. hierzu deshalb Francksen, Staatsrat, S.27f.

${ }^{3}$ Antwortschreiben Napoleons an Joachim Murat vom 4. April 1806, AN, 31 AP 24. 
nach wie vor als politische Manövriermasse bzw. als Kompensationsobjekte betrachtete und sich deshalb in seinem Handlungsspielraum nicht einschränken lassen wollte.

(2) Nachdem Prinz Murat mit seinen anfänglichen Verfassungsplänen gescheitert war, nahm er im Rahmen der territorialen Erweiterung des Großherzogtums im Januar 1808 einen neuen Anlauf in der Verfassungsfrage. Wie im Frühjahr 1806 wollte er die Organisations- und Integrationsaufgaben, die mit den Gebietsgewinnen verbunden waren, auf dem Weg der Verfassungsgebung lösen. Zu diesem Zweck befahl er Finanzminister Agar am 23. Januar 1808 darauf ist bereits hingewiesen worden -, die Staatsräte unverzüglich damit zu beauftragen, der Konstitutionsakte des Königreichs Westfalen all jene Maximen zu entlehnen, die sie als geeignet für das Großherzogtum betrachteten ${ }^{4}$. Außerdem verlangte er von seinem Finanzminister die Ausarbeitung eines Verfassungsentwurfs ${ }^{5}$. Die von ihm beabsichtigten Veränderungen sollten noch vor Inbesitznahme der dem Großherzogtum anheimgefallenen Gebiete, d.h. noch vor dem 1. März 1808 erfolgen, pour qu'à cette époque, je puisse mettre en activité dans les pays réunis, l'administration et la contribution du Grand Duché, avec les changemens que je vous ai chargé de puiser dans la Constitution de Westphalie 6 . Deshalb forderte Murat von Agar, die Reformvorschläge bis zum 15 . Februar 1808 vorzulegen?

Im Unterschied zu 1806 waren die Voraussetzungen für Murats Vorhaben zu Beginn des Jahres 1808 günstig. Napoleon strebte danach, die Rheinbundstaaten strukturell zu vereinheitlichen und nach französischem Vorbild zu gestalten. Im Jahr zuvor hatte überdies das Königreich Westfalen eine Konstitutionsakte erhalten, die der Düsseldorfer Regierung bei der Ausarbeitung eines Entwurfs als Leitfaden dienen konnte und durch welche gleichzeitig mehr Erfolg für die Durchsetzung eines vergleichbaren Unternehmens in Berg zu erwarten war. Immerhin hatte Napoleon die Ausarbeitung der westfälischen Verfassung selbst initiiert ${ }^{8}$.

Finanzminister Agar verschwieg seinem Landesherrn nicht, daß er es ablehnte, dem Großherzogtum einen verfassungsrechtlichen Rahmen zu geben. Zwar betrachtete er die einzelnen Bestandteile der westfälisch-französischen Verfassung für das Großherzogtum als zweckmäßig. Doch zog er es vor, die Inhalte der westfälischen Konstitutionsakte in Form von gesetzlichen Einzelmaßnahmen nach und nach in das Land zu importieren, statt sie im Rahmen eines Verfassungsaktes en bloc zu übernehmen. Den 1. Januar 1809 betrachtete er als realistischen Termin, um mit den Veränderungen zu beginnen. Agar sprach sich aus mehreren Gründen gegen den Erlaß einer Verfassung aus.

\footnotetext{
${ }^{4}$ Joachim Murat an Finanzminister Agar in seinem Schreiben vom 23. Januar 1808, ADD, Fonds Mosbourg, 100 MI 114 R2.

5 Ibid.

6 Joachim Murat an Agar in seinem Schreiben vom 28. Januar 1808, AN, 31 AP 17.

7 Ibid.

8 Vgl. dazu Francksen, Staatsrat, S. 51 .
} 
Zum ersten waren seiner Meinung nach verschiedene Punkte der westfälischen Verfassung im Großherzogtum bereits verwirklicht. Zum zweiten befürchtete er, der Erlaß einer kodifizierten Verfassung führe zu erheblichen Steuerausfällen. Zum dritten sah er in dem Verfassungsakt eine potentielle Gefahr für die Integrität des Großherzogtums.

En publiant une constitution, warnte er Murat, vous ferez connaitre tout ce que vous voulez détruire \& tout ce que vous voulez créer; cependant, le plus grand nombre des institutions éxistantes, ne pourront, dans le fait, ni cesser, ni être remplacées que successivement. Mais dès l'instant où vous les aurez frappées, dès l'instant où leur chûte sera certaine, elles perdront leur crédit \& leur énergie, elles ne rempliront plus qu'imparfaitement leur objet \& les institutions nouvelles n'étant pas encore formées, plusieurs parties de l'administration publique demeureront languissant \& négligées [sic] ${ }^{9}$.

Als Beweis für die Stichhaltigkeit seiner Position wie auch als Argument für die von ihm angebotene Handlungsalternative machte sich der Finanzminister die Probleme zunutze, die in Westfalen seit Inkrafttreten der Konstitution entstanden waren.

Témoins des embarras qu'éprouvent les Westphaliens, pourqoui ne cherchions nous pas à profiter de l'expérience qu'ils vont faire, des règlemens utiles que leur gouvernement publiera, des erreurs même qui pourraient leur échapper! Vos sujets jouiraient alors de tous les bienfaits de la législation nouvelle, sans avoir à souffrir les inconvéniens \& les préjudices qu'entrainent les changemens brusques ${ }^{10}$.

$\mathrm{Da}$ Agar in der Verfassungsfrage eine andere Ansicht vertrat als der Monarch, war wohl auch ein Grund dafür, daß er den Düsseldorfer Staatsrat zwar in die landesherrlichen Verfassungspläne einweihte und ihn über $\mathrm{Mu}-$ rats Absicht informierte, das Großherzogtum so weit wie möglich nach westfälischem Beispiel zu gestalten, aber aus der Mitte des Staatsrats zwei Kommissionen nur mit der Bearbeitung der von Murat benannten konkreten Sachgebiete betraute ${ }^{11}$. Murat ließ er jedoch in einem anderen Glauben. Die Staatsräte hätten sich, teilte er dem Prinzen Anfang Februar 1808 mit, einhellig für die Übernahme der westfälischen Verfassung ausgesprochen. Sie seien nicht nur der Auffassung, qu'elle convenait à vos états, mais encore qu'elle serait pour tous vos sujets [...] un bienfait inappréciable ${ }^{12}$. Den von Murat gewünschten Verfassungsentwurf, der eigentlich Sache des Staatsrats hatte sein sollen, arbeitete Agar selbst aus und ließ ihn Murat zusammen mit seinen Bedenken gegen eine kodifizierte Verfassung zukommen. Die von ihm aus-

9 Antwortschreiben Agars an Joachim Murat vom 2.Februar 1808, ADD, Fonds Mosbourg, $100 \mathrm{MI} 119$.

10 Ibid.

11 Protokoll der Staatsratssitzung vom 2. Februar 1808, HStAD, GB, Nr.249.

12 Schreiben Agars an Joachim Murat vom 9. Februar 1808, ADD, Fonds Mosbourg, 100 MI 119; vgl. auch Francksen, Staatsrat, S. 53; RoB, Regierungsakten des Großherzogtums Berg, S. 14. 
gearbeitete Konstitution deckte sich grosso modo mit dem Inhalt der westfälischen Vorlage ${ }^{13}$.

Prinz Murat hielt Agars Zweifel und Einwände nicht für gerechtfertigt. Er belehrte seinen Finanzminister am 13. Februar 1808, daß er zur Vermeidung der von Agar antizipierten Gefahren gar nicht die vollständige Übernahme angeordnet, sondern nur befohlen habe, jene Elemente der westfälischen Verfassung zu übernehmen, die für das Großherzogtum geeignet seien ${ }^{14}$. Zumindest aber ließ sich der Prinz davon überzeugen, den Termin für die Durchsetzung seines Vorhabens zu verschieben. Die Verfassung sollte zwar erarbeitet, doch erst im Verlauf des Jahres in Kraft gesetzt werden. Als Stichtag war, wie von Agar gewünscht, der 1.Januar 1809 geplant $^{15}$.

Die Pariser Zentrale nahm zu dem von Agar ausgearbeiteten Verfassungsentwurf, den Murat - bezeichnenderweise als Arbeit des Staatsrats ${ }^{16}$ - noch unmittelbar vor seiner Ernennung zum Generallieutnant in die französische Hauptstadt übersandte, um ihn von Napoleon genehmigen zu lassen, nicht Stellung. Anscheinend hatte der französische Kaiser ungeachtet der veränderten politischen Rahmenbedingungen seine Einstellung in der bergischen Verfassungsfrage nicht geändert und lehnte nach wie vor eine geschriebene Verfassung für das Großherzogtum ab. Murat verfolgte seine konstitutionellen Absichten in den Folgemonaten nicht weiter, zumal er in militärische Aktionen verwickelt wurde ${ }^{17}$. Auch dieses Mal kam es deshalb nicht zum Erlaß einer kodifizierten Verfassung. Finanzminister Agar sah sich im nachhinein durch Vorgänge in Westfalen darin bestätigt, daß es richtig gewesen war, das Großherzogtum nicht mit einer Verfassung auszustatten. In Kassel machten nämlich die Reichsstände von ihrem Ablehnungsrecht Gebrauch gemacht und verwarfen im August 1808 in großer Mehrheit ein vom westfälischen Staatsrat vorgelegtes Grundsteuergesetz. Mehr als zwei Drittel der Mitglieder stimmten gegen die Vorlage. Cet exemple ne peut que faire sentir d'avantage, schloß der Finanzminister daraus, wie weise es gewesen sei, das Verfassungsprojekt zu vertagen ${ }^{18}$.

13 Antwortschreiben Agars an Joachim Murat vom 2. Februar 1808, ADD, Fonds Mosbourg, $100 \mathrm{MI}$ 119. Es handelt sich hierbei um den in der Geschichtswissenschaft verlorengeglaubten Verfassungsentwurf, den Murat wenig später, wie weiter unten noch ausgeführt werden wird, Napoleon zukommen ließ. Agar stellte diesen Entwurf, anders als Rob vermutete, nicht als Projekt des Staatsrats dar, sondern machte vielmehr von Anfang an klar, daß es sich um seine eigene Arbeit handelte. Vgl. FrancksEn, Staatsrat, S. 54; RoB, Regierungsakten des Großherzogtums Berg, S.15; ENGELBRECHT, Modellstaat, S. 256.

14 Schreiben Joachim Murats an Finanzminister Agar vom 13. Februar 1808, AN, 31 AP 17.

15 Schreiben Joachim Murats an Agar vom 18. Februar 1808, ADD, Fonds Mosbourg, 100 MI 114 R2.

16 Schreiben Joachim Murats an Napoleon vom 18. Februar 1808, abgedruckt in: Lettres et documents, Bd. 5, Nr. 3017, S. 279; vgl. auch das Schreiben Joachim Murats an Agar vom 18. Februar 1808, ADD, Fonds Mosbourg, 100 MI 114 R2.

17 Ende Februar 1808 schickte Napoleon ihn als Generallieutnant nach Spanien. Vgl. Dufraisse, Kerautret, France, S.127, 289.

18 So Finanzminister Agar in seinem Schreiben an Joachim Murat vom 15. August 1808, ADD, Fonds Mosbourg, 100 MI 119. Mit den Vorgängen im Königreich Westfalen be- 
(3) Die Verfassungsfrage kam abermals zur Sprache, nachdem der Kaiser am 3. März 1809 das Großherzogtum seinem Neffen übertragen und selbst die Regentschaft übernommen hatte. Anstoß dazu gab eine aus Regierungsbeamten, Ständevertretern und Kaufleuten des Großherzogtums bestehende und von den beiden Düsseldorfer Ministern genehmigte zwölfköpfige Deputation, die sich anläßlich des Thronwechsels Ende März 1809 zu einer Audienz bei Herzog Bassano nach Paris begab ${ }^{19}$. Bei ihrem Treffen mit dem französischen Minister-Staatssekretär am 31. März 1809 brachten die Abgeordneten neben anderen Wünschen auch die Bitte vor, Napoleon möge für das Großherzogtum eine Verfassung erlassen. Sie legten zu diesem Zweck einen Verfassungsentwurf vor, der inhaltlich weitgehend der westfälischen Konstitutionsakte entsprach ${ }^{20}$. Die Initiative der Delegierten markierte in der Verfassungsdiskussion einen Einschnitt, denn damit brachten erstmals einheimische Kräfte ihr Interesse an der politischen Stabilisierung des Großherzogtums offen zum Ausdruck. Sie wünschten, daß der politische Schwebezustand ihres Landes beseitigt wurde und dieses einen verbindlichen und vor allem dauerhaften konstitutionellen Rahmen erhielt.

Die Deputierten verbanden mit ihrem Unternehmen einige Hoffnung. Es sei möglich, schrieb Graf von Westerholt, einer der Deputierten, am 8. April 1809 nach Düsseldorf, que cette deputation ne soit pas sans utilité21. Allerdings verfügten die Abgeordneten über keinerlei Druckmittel, um ihren Forderungen Nachdruck zu verleihen, und diejenigen Beamten, die den Erlaß einer Konstitution im Großherzogtum befürworteten oder unterstützten, besaßen auch nicht genügend Einfluß, um den Wunsch der Abordnung höhernorts wirksam zu fördern. Überdies hatte die Deputation keinen günstigen Zeitpunkt für ihr Unternehmen gewählt. Seit dem Vorjahr keimten an mehreren Stellen des französischen Empire Unruhen. Diese nahmen nicht nur die Aufmerksamkeit der Pariser Regierung in Anspruch, sondern waren zudem dem Verfassungsgedanken nicht förderlich. Insbesondere die Entwicklungen im Königreich Westfalen zeugten nicht vom Erfolg der französischen Verfassungspolitik im Rheinbund und sprachen in gewisser Weise für die im Großherzogtum verfolgte Reform der kleinen Schritte.

Stellt man diese innen- und außenpolitischen Rahmenbedingungen in Rechnung, überrascht es nicht, daß sich die Verfassungswünsche der Delegierten nicht erfüllten. Zwar sah es im Herbst 1809 kurzzeitig so aus, als wolle man in

schäftigen sich HECKER, Konstitutionalismus, S. 152f., BrAKENSIEK, Reichsstände, S.233f.; Herbert Obenaus, Die Reichsstände des Königreichs Westfalen, in: Francia 9 (1981) S. 299-329, hier S.315f.

19 Siehe das von Beugnot und Nesselrode unterzeichnete Protokoll über die Verrichtungen der im März 1809 nach Paris abgesandten Deputation des Großherzogtums Berg vom 24. März 1809, HStAD, GB, Nr. 47.

20 AN, AF IV 365, plaq. 2666. Der Entwurf ist nicht signiert. Er ist auf den 8. März 1809 datiert.

21 Von Westerholt in seinem Schreiben aus Paris vom 8. April 1809, HStAD, GB, Nr. 34. 
Paris der Bitte der Deputierten doch noch nachkommen. Jedenfalls teilte Herzog von Bassano Beugnot Anfang Oktober 1809 mit, Napoleon sei geneigt, für das Großherzogtum eine Konstitution zu stiften, und bat ihn, dies bei der Erarbeitung seiner Vorschläge zur Reorganisation der Regierungsbehörden zu berücksichtigen 22 . Wenig später forderte er Staatsrat Hazzi konkret dazu auf, schnellstmöglich einen Verfassungsentwurf für das Großherzogtum vorzulegen. Die Konstitution sei de première nécessité pour ce pays, et la base de tout, begründete er diesen Schritt ${ }^{23}$. Aber ernsthafte Verfassungsdiskussionen oder konkrete Planungen kamen auch dieses Mal nicht in Gang. Beugnot trat gegen einen Verfassungserlaß ein, weil er wie sein Vorgänger den Staat bereits für ausreichend verfaßt hielt und der Meinung war, qu'une constitution qui est un fait est quelque chose, et que celle qui est un livre, n'est encore rien ${ }^{24}$. Der Verfassungsentwurf, den Staatsrat Hazzi auf Grundlage der westfälischen Konstitutionsakte erarbeitet und vorgestellt hatte, wurde ad acta gelegt, ohne näher erörtert oder geprüft zu werden ${ }^{25}$. Ebenso scheiterte Hazzis Versuch, anläßlich der Ernennung Roederers zum Minister-Staatssekretär im Herbst 1810 die seit mehr als drei Jahren erwartete Verfassungskodifikation zu erwirken ${ }^{26}$.

Eine definitive Entscheidung fiel jedoch auch nicht. Napoleon war sich durchaus der Tatsache bewußt, daß durch die französische Herrschaft unter den Einwohnern das Bedürfnis nach einer geschriebenen Verfassung geweckt worden war, wobei das ganze Ausmaß dieser Entwicklungen erst nach dem Zusammenbruch des Großherzogtums spürbar wurde. Um das Vertrauen dieser Einwohner nicht zu verspielen, hielt er die Verfassungsfrage daher absichtlich offen - mehr noch: Bei seinem Besuch der bergischen Hauptstadt Anfang November 1811 stellte er dem Land sogar persönlich eine Verfassung in Aussicht ${ }^{27}$.

Der französische Kaiser hatte mit seiner Hinhaltetaktik durchaus Erfolg. Im Großherzogtum gab man in der Tat den Glauben an den Erlaß einer Kon-

22 Ein Auszug des herzoglichen Schreibens an Beugnot vom 1. Oktober 1809 befindet sich in der Akte AN, AF IV 642, plaq. 5099; vgl. auch Kap. III, 1.2.

23 Das geht aus Hazzis Antwortschreiben an den Minister-Staatssekretär vom 18. Januar 1810 hervor, AN, AF IV 1225. Demgegenüber vertritt RoB, Regierungsakten des Großherzogtums Berg, S.16, die Meinung, Hazzi habe den Verfassungsentwurf eigenmächtig verfaßt und nach Paris gesandt.

24 Beugnot in seinem Mémoire sur l'organisation du gouvernement dans le Grand Duché an Maret vom 10. November 1809, AN, AF IV 642, plaq. 5099.

25 Konstitutionsentwurf des Staatsrats Hazzi an Herzog Bassano vom 18. Januar 1810, AN, AF IV 1225.

26 Tout y est provisoire, beklagte Hazzi in seinem Brief an den soeben ernannten MinisterStaatssekretär Roederer vom 5.Oktober 1810, tout en souffrance: les objets d'un intérêt général comme ceux d'un intérêt particulier. Als Vorschlag überreichte er ihm den Konstitutionsentwurf, den er Anfang des Jahres Herzog von Bassano vorgestellt hatte. Vgl. AN, AF IV 642, plaq. 5099.

27 Protokoll der Sitzung Napoleons mit dem französischen Außenminister Maret, dem französischen Minister-Staatssekretär Daru sowie Roederer, Beugnot und Nesselrode vom 3. November 1811, AN, 29 AP 36. 
stitution während der französischen Herrschaft nicht auf. Im Frühjahr 1811 etwa kündigte Präfekt Romberg seinen schon seit längerem geplanten Rückzug aus dem politischen Leben an, weil er vom baldigen Erlaß einer Verfassung ausging ${ }^{28}$. Im Dezember desselben Jahres erinnerte Nesselrode den großherzoglich-bergischen Minister-Staatssekretär an das Versprechen, das Napoleon anläßlich seiner Reise nach Düsseldorf einen Monat zuvor gemacht hatte ${ }^{29}$, und am 24. September 1813 wandte sich Roederer mit der Zuversicht an den Kaiser, daß die Erwartung der Einwohner auf eine Verfassung nach Beendigung des Krieges erfüllt werde ${ }^{30}$. Kehrseite dieser Politik war allerdings, daß sich der französische Landesherr durch das Hinausschieben der Verfassungsfrage vor allem bei denjenigen Einwohnern diskreditierte, auf die er bei der Konsolidierung seiner Herrschaft am Niederrhein besonders angewiesen war: den Notabeln.

\section{Verfassungsentwicklung}

\subsection{Politische Mitsprache}

\subsubsection{Neuordnung der gesamtstaatlichen Repräsentation}

Eines der vorrangigen verfassungspolitischen Anliegen Murats nach dem Scheitern seiner konstitutionellen Pläne im Frühjahr 1806 war die Umgestaltung der Regierungsspitze. Nur wenige Wochen, nachdem Napoleon sein Verfassungsprojekt abgelehnt hatte, leitete er erste Schritte dazu in die Wege. Seine Bestrebungen richteten sich in diesem Rahmen vor allem darauf, die Leistungsfähigkeit der Exekutive zu verbessern. Der Umbau der vorgefundenen Oberbehörden diente dieser Absicht. Außerdem bemühte sich der Prinz darum, seine Herrschaft flächendeckend durchzusetzen. Zu diesem Zweck wollte er die in der tradierten Verfassung verankerten Stände, die kraft eigenen Rechts bestanden und ihm seine Monopolstellung zumindest theoretisch immer noch streitig machten, aus der Staatsspitze verdrängen. Anfangs plante der Prinz die ersatzlose Streichung der Ständekörperschaft. Nach dem Erlaß einer Verfassungsurkunde im Nachbarstaat Westfalen entschied er sich dazu, die Stände durch ein dem westfälischen Beispiel nachgebildeten Repräsentativorgan zu ersetzen. Die Einrichtung einer solchen Körperschaft stand letztlich in keinerlei Widerspruch zu seinen Monopolisierungsbestrebungen, denn es ging ihm nicht darum, ein echtes parlamentarisches Vertretungsorgan zu schaffen. Das geplante Kollegialorgan sollte keinerlei Recht zur Mitsprache oder Kontrolle erhalten. Deshalb wird die französische Verfassungspolitik in

28 Schreiben des Präfekten Romberg an den Generalprokurator Sethe vom 30. April 1811, StAM, Nachlaß Giesbert von Romberg A, Nr.11.

29 AN, 29 AP 39.

30 Schreiben Roederers an den Kaiser vom 24. September 1813, AN, AF IV 711, plaq. 5697. 
der historischen Forschung bis heute nur als »Scheinkonstitutionalismus« bezeichnet $^{31}$.

Wegen der hohen Bedeutung der Oberbehörden für die Herrschaftssicherung kümmerte sich die französische Regierung zuerst um den administrativen Umbau, ehe sie sich mit der Ständefrage und dem damit korrelierenden Problem einer gesamtstaatlichen Repräsentation beschäftigte. Immerhin stellten die Regierungsbehörden das Ausführungsorgan des landesherrlichen Willens dar. Hinzu kam, daß der französische Monarch aufgrund der fehlenden dynastischen Legitimation zu Beginn seiner Herrschaft dazu genötigt war, die Stände weiterhin zu dulden. Eine solche Haltung lag paradoxerweise wegen der geplanten Reformen selbst nahe, denn auf französischer Seite glaubte man die Chancen für die Durchsetzung von Reformen in der Bevölkerung zu verbessern, wenn man die ständischen Repräsentationsorgane bestehen lie ${ }^{32}$. Verfassungsrechtliche Maßnahmen erübrigten sich anfänglich auch deshalb, weil die Neuerungen im administrativen Bereich gleichzeitig ein Hebel zur Stärkung der Exekutive waren. Ohne in ihrer Existenz in Frage gestellt zu werden, verloren die Stände nämlich infolge der Umbaumaßnahmen in der Staatsverwaltung faktisch noch mehr an Daseinsberechtigung. Einerseits erfolgte die Reformierung der Landesbehörden ganz ohne ständische Beteiligung, und auch bei der Personalrekrutierung wurde auf ständische Reservatrechte keinerlei Rücksicht mehr genommen. Hierin setzte die Regierung die Politik der meisten Vorläuferterritorien auf dem bergischen Gebiet fort, die Stände in praxi auf eine politische Randexistenz zu reduzieren, ohne sie völlig aufzuheben. Andererseits wurde mit dem Staatsrat eine Institution geschaffen, welche die tradierten Mitsprachemöglichkeiten der ständischen Landeskollegien übernahm - das Steuerbewilligungsrecht ausgenommen - und diese weitgehend überflüssig machte.

Die Regierung dachte zum ersten Mal ernsthaft über eine mögliche Reformierung der Ständeverfassung nach, als sie sich im Frühjahr 1806 darum bemühte, die Steuererhebung zu vereinheitlichen, um die Einnahmen des Fiskus zu verbessern. Hierbei wurde sie nämlich unweigerlich mit der ständischen Mitsprache in der Finanzpolitik konfrontiert ${ }^{33}$. Innenminister Nesselrode,

31 Der Begriff stammt von HUBER, Deutsche Verfassungsgeschichte, S.88; siehe auch KLUETING, Großherzogtum Frankfurt, S.378f. Kritische Auseinandersetzung mit dem Huberschen Verdikt: Elisabeth FEHRENBaCH, Verfassungs- und sozialpolitische Reformen und Reformprojekte in Deutschland unter dem Einfluß des napoleonischen Frankreich, in: Berding, UllmanN, Revolution und Restauration, S. 65-90, hier S.71f.; Press, Landstände, S.143f.; Ullmann, Finanzreformen, S. 125; OwZar, Verfassungspolitik, S. 312.

32 Brief Joachim Murats an Napoleon vom 28. März 1806, abgedruckt in: Lettres et documents, Bd.4, Nr. 2289, S. 184f., hier S. $186 f$.

33 Die Erhöhung der Staatseinnahmen war notwendig, um die Reformpolitik zu finanzieren, die fürstlichen Einkünfte zu heben und die finanziellen Leistungen an Frankreich sicherzustellen. Zum Zusammenhang von Finanzen und Reformpolitik: Hans-Peter ULLMANN, Staatsschulden und Reformpolitik. Die Entstehung moderner öffentlicher Schulden in Bayern und Baden 1780-1820, Göttingen 1986; DERS., Der Staatskredit im Rheinbund: 
den Agar damit beauftragt hatte, Reformvorschläge in dieser Frage zu machen, plädierte dafür, die in den einzelnen Provinzen vorhandenen ständischen Kollegien zu einer Körperschaft zu vereinigen und die Ständemitglieder aus ihrer regionalen Verankerung zu lösen. Die Mitglieder der Stände sollten nicht mehr als Vertreter eines Territoriums agieren, sondern als $\mathrm{Re}$ präsentanten des gesamten Landes. Darüber hinaus empfahl er, das Kollegium der landtagsfähigen Städte durch Elberfeld, Solingen, Barmen und Mülheim sowie durch die bisher nicht repräsentierten Ämter zu ergänzen. Die Wahl der Amtsvertreter war an den Zensus geknüpft ${ }^{34}$. Hiermit wollte Nesselrode den wirtschaftlichen Entwicklungen gerade im ehemaligen Herzogtum Berg Rechnung tragen und zugleich dem aus den Reihen der Bevölkerung seit langem gehegten und erst jüngst wieder vorgebrachten Wunsch nach veränderten Repräsentationsverhältnissen im Kollegium der Städte nachkommen ${ }^{35}$. Während der Innenminister mit der Verschmelzung der regionalen Ständekörperschaften durchaus modern dachte, blieben seine Vorstellungen doch altständischen Ordnungsmustern verhaftet. Er sprach sich nämlich grundsätzlich dafür aus, innerhalb des vereinigten Ständekorpus an der Gliederung der Stände in jeweils eine Körperschaft für den klevischen und eine für den bergischen Adel sowie jeweils eine für die Städte und Ämter der beiden Herzogtümer festzuhalten.

Finanzminister Agar stimmte mit Nesselrode im Grundsatz darin überein, daß die in den einzelnen Provinzen vorhandenen ständischen Kollegien zu einer Körperschaft zusammengeschmolzen werden mußten, war aber, was die weiteren Aufgaben der ständischen Vertretungen, ja, das künftige Repräsentativsystem überhaupt betraf, anderer Meinung. Er setzte sich dafür ein, die ständischen Organe vollständig aus ihrer regionalen Verankerung zu lösen und zu einer beide Herzogtümer vertretenden Körperschaft zu vereinen. Nur so war für ihn gewährleistet, daß die Ständemitglieder nicht damit fortfuhren, primär die Interessen einzelner Territorien zu vertreten. Eine wirkliche Volksvertretung strebte aber auch er nicht an, denn ebenso wie sein Kollege sprach er sich dafür aus, bei der personellen Zusammensetzung des Repräsentativorgans am ständischen Prinzip festzuhalten. Die Kollegien des Adels und der Städte sollten erhalten bleiben. Die Vertretung der Städte wollte er durch Repräsentanten der Ämter erweitern ${ }^{36}$.

Bayern, Württemberg und Baden im Vergleich, in: Francia 10 (1982) S.327-343. Dem Ansatz Ullmanns verpflichtet ist MURK, Reichsterritorium.

34 Der Entwurf Nesselrodes ist dem Schreiben Agars an Joachim Murat vom 18. Juni 1806 beigefügt, ADD, Fonds Mosbourg, 100 MI 116.

35 Beispielsweise hatte der Elberfelder Magistrat mit Hinweis auf die gewachsene Bedeutung Elberfelds in Handel und Industrie Mitte April 1806 darum gebeten, Elberfeld in die Ständeversammlung aufzunehmen. Eingabe des Elberfelder Magistrats an den Großherzog vom 14. April 1806, HStAD, GB, Nr. 35.

36 Schreiben Agars an Joachim Murat vom 18. Juni 1806, ADD, Fonds Mosbourg, 100 MI 116. 
Agars Forderungen waren nicht neu. Bei der Bestandsaufnahme der Herzogtümer Kleve und Berg im März 1806 hatte der französische Staatsdiener bereits auf die Reformbedürftigkeit der Ständeverfassung aufmerksam gemacht und den Zusammenschluß der ständischen Körperschaften zu einem aus zwei Kammern bestehenden Vertretungsorgan gefordert. Ebenso war er mit Blick auf die Erwartungen der Einwohner schon zu diesem Zeitpunkt dafür eingetreten, die Repräsentationsverhältnisse innerhalb des Kollegiums der Städte zu modifizieren, zumal er eine solche Maßnahme auch unter außenpolitischen Gesichtspunkten als werbewirksam betrachtete. Une représentation égale et régulière, erwartete er, exciterait la reconnaissance universelle et serait enviée de toutes les contrées voisines ${ }^{37}$.

Mit seinen Bedenken gegen die Empfehlungen seines Kollegen brachte der Finanzminister das Reformvorhaben zunächst zum Erliegen. Die Steuervereinheitlichung wurde vertagt. Die Ständeverfassung blieb in ihrer überlieferten Form einstweilen erhalten ${ }^{38}$. Nach den territorialen Erweiterungen des Großherzogtums im Rahmen der Rheinbundakte berief Murat sogar am 22. August 1806 die Stände kraft seiner monarchischen Vollmachten nach Düsseldorf, um sie, wie er ankündigte, die Steuerverteilung vornehmen und über das resp. Interesse dieser Provinzen, so wie auch über die Maaßregeln, wodurch sie unter eine und dieselbe Konstitution vereinigt werden können beraten zu lassen ${ }^{39}$. Allerdings leiteten ihn bei der Einberufung der Landeskollegien andere Motive, als diese erneut am politischen Entscheidungsprozeß zu beteiligen. Er wollte auf diesem Weg erreichen, daß die Stände freiwillig auf ihre Steuerprivilegien verzichteten und damit auch an der Aufbringung der Grundsteuer beteiligt werden konnten.

Murat setzte Napoleon am 24. August 1806 von seiner Entscheidung in Kenntnis, die Stände in der bergischen Hauptstadt zusammenkommen zu lassen. Er machte in diesem Zusammenhang kein Geheimnis daraus, was er mit diesem Schritt bezweckte. Er müsse zugeben, bekannte er Napoleon gegenüber, daß die Versammlung der Stände einem anderen Zweck diene und die Abstimmung über die Steuer nur ein Vorwand sei. Sein eigentliches Ziel sei, fuhr er fort, de faire adopter par les Etats la détermination de faire peser également l'impôt sur tous les biens, et de lever tous les privilèges qui peuvent y être attachés; par ce moyen toutes les propriétés et tous les domaines des Princes dépossédés payeront également, ce qui augmentera beaucoup le produit des contributions ${ }^{40}$.

${ }^{37}$ Finanzminister Agar in seinem undatierten Tableau de l'administration civile et judiciaire des duchés de Clèves et de Berg sous le régime prussien (März 1806), AN, AF IV 1225.

38 Schreiben Agars an Joachim Murat vom 18. Juni 1806, ADD, Fonds Mosbourg, $100 \mathrm{MI}$ 116.

39 Zit. nach Rudolf Goecke, Das Großherzogthum Berg unter Joachim Murat, Napoleon I. und Louis Napoleon 1806-1813. Ein Beitrag zur Geschichte der französischen Fremdherrschaft auf dem rechten Rheinufer, Köln 1877, S.52.

40 Schreiben Joachim Murats an Napoleon vom 24. August 1806, abgedruckt in: Lettres et documents, Bd.4, Nr. 2493, S. 324f., Zitat S. 325. 
Gleichzeitig ließ er dem französischen Kaiser zwei Gesetzentwürfe zur Bewilligung zukommen. Der erste richtete sich auf das, was Murat eigentlich mit der Ständeversammlung bezweckte: die Beseitigung der ständischen Steuerprivilegien. Der zweite hatte die vollständige Auflösung der ständischen Kollegien zum Ziel. Murat rechtfertigte diesen Schritt nicht, wie zu erwarten gewesen wäre, damit, daß nicht in allen dem Großherzogtum zugefallenen Gebieten landständische Vertretungen existierten. Vielmehr machte er die Stände selbst für die geplante Auflösung verantwortlich. Die Erfahrung zeige, behauptete er, daß die Stände dazu neigten, die Regierungspolitik zu blockieren ${ }^{41}$.

Der Großherzog verfolgte mit seiner Doppelstrategie, die Stände in Düsseldorf zu versammeln und parallel dazu in Paris zu beantragen, die Befreiung der Stände von der Grundsteuer aufzuheben und die Stände zu beseitigen, wohl vor allem zwei Ziele. Auf der einen Seite suchte er hierdurch einem Akzeptanz- und Vertrauensverlust seitens der Stände vorzubeugen ${ }^{42}$. Auf der anderen Seite wollte er selbst bei einem negativen Bescheid aus Paris sichergehen, das Hauptziel seiner Reformbestrebungen - die Sicherung neuer Finanzquellen - zu erreichen. Eine von ihm gefällte Entscheidung konnte die Pariser Zentrale durchaus revidieren. Die Beschlüsse der Stände mußte sie dagegen zwangsläufig akzeptieren, wollte sie ihre Glaubwürdigkeit inner- wie außerhalb des Großherzogtums nicht aufs Spiel setzen.

In Paris fanden die Pläne, die man im Spätsommer 1806 von Murat zur Genehmigung erhielt, keine Zustimmung. Außenminister Talleyrand, der die Angelegenheit im Auftrag Napoleons zu prüfen hatte, billigte weder die Aufhebung der Steuerbefreiungen noch die Beseitigung der Stände. Er war der Ansicht, als Erbmonarchie benötige das Großherzogtum einen erblichen Adel, und dessen Existenz sah er wiederum nur durch Privilegien gesichert. Außerdem sah er die Akzeptanz der neuen Herrschaft in der Bevölkerung durch die vollständige Aufhebung der Stände gefährdet, zumal die Stände seiner Meinung nach dem französischen Souverän noch keinerlei Anlaß zur Klage gegeben hatten. Neben diesen innenpolitischen Zweifeln hatte Talleyrand eine Reihe außenpolitischer Bedenken gegen die Düsseldorfer Reformvorhaben. Die Abschaffung der Grundsteuerprivilegien kollidierte seiner Meinung nach mit der Rearistokratisierungspolitik Napoleons ${ }^{43}$ und dabei ganz besonders mit dem Erlaß vom 14. August 1806, mit dem Napoleon die Majorate wieder eingeführt hatte. Darüber hinaus wurden Sinn und Inhalt des Rheinbundvertrags verletzt, denn in diesem waren die mediatisierten Fürsten in ihren Steuerprivilegien bestätigt worden. Ce serait éluder et non pas remplir l'intention du Traité, erläuterte er, qui, pour adoucir les regrets des Princes et

41 Ibid.

42 Wie wichtig ihm die förmliche Zustimmung der ständischen Körperschaften war, zeigt sich auch daran, daß er die Stände der wenig später an das Großherzogtum gefallenen Stifte Essens und Werdens ebenfalls dazu aufforderte, ihre Entprivilegisierung förmlich zu beschließen. Brand, Geschichte, S. 99f.

43 Vgl. BERDing, Gesellschaftsgedanke. 
Comtes et les consoler de la perte de leur Souveraineté, a voulu bien évidemment qu'ils conservassent tous les privilèges et toutes les prérogatives qui n'étaient pas absolument incompatibles avec leur nouvelle situation ${ }^{44}$. Was die Aufhebung der Stände betraf, so befürchtete der französische Außenminister, $\mathrm{da} B$ dadurch Kettenreaktionen in den übrigen deutschen Staaten ausgelöst wurden - insbesondere wegen der Vorbildfunktion, die das Großherzogtum innerhalb des Rheinbunds erfüllte. Murat befinde sich in einer besonderen Situation, erklärte er diesem in seinem Schreiben vom 15. September 1806, denn l'influence de ses actes ne saurait être bornée à l'intérieur de ses Etats. Les Princes ses voisins seront excités par son exemple ${ }^{45}$. Talleyrand legte Murat deshalb nahe, sich darauf zu beschränken, exzessive Privilegien des Adels zu beschneiden ${ }^{46}$. Was die Auflösung der Stände betraf, empfahl er ihm, diese auf einen späteren Zeitpunkt zu verschieben oder aber die Provinzial- in Generalstände für das gesamte Großherzogtum umzuwandeln ${ }^{47}$.

Bevor das Antwortschreiben des französischen Außenministers in Düsseldorf eintraf, war die Angelegenheit jedoch bereits im Interesse des französischen Landesherrn geregelt worden. Anfang September 1806 hatten sich die Stände in Düsseldorf zusammengefunden und freiwillig auf ihre Steuerprivilegien verzichtet - wohl nicht zuletzt, um ihre Existenz zu retten. Bei dieser Gelegenheit hatten sie sich ebenfalls, wie von ihrem Landesherrn gewünscht, zu einer gemeinsamen Körperschaft zusammengeschlossen ${ }^{48}$. Murat konnte die Pariser Regierung daher vor ein Fait accompli stellen. J'ai l'honneur de mettre sous les yeux de V.M., teilte er Napoleon am 11. September 1806 mit, le procèsverbal de l'ouverture et des séances de mes états. Elle y verra peut-être avec plaisir leur première démarche et le bon esprit qui les anime $e^{49}$.

Für die Stände zahlte sich die Kompromißbereitschaft gegenüber dem neuen Landesherrn nicht aus. Der Großherzog löste seine Ankündigung, die Stände an der Reformpolitik zu beteiligen, in der Folgezeit ebensowenig ein wie sein Versprechen, sie als Ganzes noch einmal zu versammeln. Lediglich im Frühjahr 1807 wurden die von den ständischen Korporationen ernannten

44 Undatierter Bericht Talleyrands an den französischen Kaiser (Sommer 1806), Archives du ministère des Affaires étrangères, Correspondance politique, Allemagne, petites principautés, Vol. 12.

45 Antwortschreiben Talleyrands an Joachim Murat vom 15. September 1806, ibid.

46 Ibid.

47 Ibid.

48 Eine Alternative hatten sie wohl ohnehin nicht, sofern sie ihre Existenz nicht in Gefahr bingen wollten. Immerhin hatte der neue Landesherr durch seine bisherige Politik keinen Zweifel daran gelassen, daß er noch weniger als seine Vorgänger dazu bereit war, sie am politischen Leben zu beteiligen. Vgl. den Beschluß der Landstände vom 9. September 1806, HStAD, GB, Nr. 12404; ferner GoEcKe, Großherzogthum, S.53f.

49 Schreiben Joachim Murats an Napoleon vom 11. September 1806, abgedruckt in: Albert Lumbroso (Hg.), Correspondance de Joachim Murat. Chasseur à cheval, général, maréchal d'empire, grand-duc de Clèves et de Berg (Juillet 1791-Juillet 1808), Turin 1899, Nr.172, S. 147. 
Deputierten noch einmal in der Hauptstadt versammelt, um die Steuerverteilung auf die Provinzen, Ämter und Kommunen vorzunehmen ${ }^{50}$. Einige Landeskollegien unternahmen zwar in der Folgezeit mehrfach Anstrengungen, um politische Entscheidungen zu beeinflussen, wobei hierbei namentlich jene Organe Partizipationsansprüche stellten, die sich in vorfranzösischer Zeit noch einen gewissen Einfluß auf die Politik bewahrt hatten. Die Ständedeputierten der ehemaligen Grafschaft Bentheim etwa verlangten am 24. Februar 1807, daß bei der Ausarbeitung eines allgemeinen Gesetzbuches für das Großherzogtum die verfassungsmäßige ständische Mitarbeit nicht aus den Augen gelassen werde ${ }^{51}$. Die Stände Münsters baten am 26. Juni 1808 darum, Deputierte in den Staatsrat entsenden zu dürfen, um an den Beratungen teilzunehmen, die die Gesetzgebung und Verfassung ihres Landes berührten ${ }^{52}$. Die Stände der Mark wollten mit aller Gewalt s'assembler, discuter; s'opposer, monierte Beugnot in der zweiten Hälfte des Jahres $1808^{53}$. Sämtliche Bemühungen scheiterten jedoch, weil die Minister keine ständische Mitsprache in den Regierungsgeschäften duldeten ${ }^{54}$.

$\mathrm{Zu}$ Beginn des Jahres 1808 bahnte sich in der Ständefrage ein Kurswechsel an. Im Rahmen seiner Pläne, dem Großherzogtum eine der westfälischen Konstitutionsakte entlehnte Verfassung zu geben, nahm Prinz Murat abermals die verfassungsrechtliche Regelung des Gegenstandes in Angriff. Seine Vorstellungen über den zu beschreitenden Reformweg hatten sich seit seinen Bemühungen im Jahre 1806 nachhaltig geändert. Während er im Sommer 1806 darauf gedrängt hatte, die Kollegien ersatzlos aufzuheben, beabsichtigte er jetzt, die altständischen Körperschaften des Großherzogtums durch eine neue Repräsentativkörperschaft zu ersetzen. Inhaltlich wollte er dabei dem französisch-westfälischen Beispiel folgen. Aufgaben und personelle Zusammensetzung des neuen Vertretungsorgans sollten dem Vorbild des Königreichs entsprechen. Murat kam allerdings auch in diesem Punkt nicht so weit, seine Reformabsichten zu verwirklichen. Da die Errichtung einer Repräsentativkörperschaft an den Erlaß einer kodifizierten Verfassung gebunden war, hing sie zwangsläufig in der Schwebe, solange in der Verfassungsfrage keine defini-

50 Schreiben Agars an Joachim Murat vom 7. Februar 1807, ADD, Fonds Mosbourg, 100 MI 118; zur Einberufung der Ständedelegierten vgl. auch GoECKE, Großherzogthum, S. $54 \mathrm{f}$.

51 Antrag der Bentheimer Deputierten, Soury und Neuerfeld, vom 24. Februar 1807, HStAD, GB, Nr. 92.

52 Schreiben Agars an Joachim Murat vom 6. Juli 1808, ADD, Fonds Mosbourg, $100 \mathrm{MI}$ 119.

53 Undatiertes Schreiben Beugnots an den ehemaligen Finanzminister Agar, AN, 40 AP 15.

54 Beispielsweise erkannte Agar die Forderungen der ständischen Vertreter Münsters nicht an, weil die Zusammenkunft der Stände von der Regierung nicht einberufen worden war, sondern widerrechtlich stattgefunden hatte. Darüber hinaus wies er darauf hin, daB die Rechte, die die französische Militärregierung den Ständen des Fürstentums zugestanden habe, mit dem Ende der militärischen Okkupation erloschen seien. Schreiben Agars an Joachim Murat vom 6. Juli 1808, ADD, Fonds Mosbourg, 100 MI 119. 
tive Entscheidung gefallen war. Die wiederholten Erinnerungen Agars, Murat möge die Ständefrage endlich klären, änderten daran nichts ${ }^{55}$.

Als die Herrschaft im Sommer 1808 auf Napoleon überging, standen zunächst andere Aufgaben im Vordergrund als die von Murat geplante Schaffung eines gesamtstaatlichen Repräsentativorgans. Dennoch wurde die seit mehr als zwei Jahren ungeklärte Ständefrage nur wenige Monate nach dem Thronwechsel gelöst. $\mathrm{Daß}$ es dazu kam, hatte die Regierung nicht direkt veranlaßt. Vielmehr handelte es sich um eine Folge bzw. Begleiterscheinung der französischen Reformpolitik im administrativen Bereich. Anlaß dazu gab der Transfer des französischen Präfektursystems auf das Großherzogtum Berg im Dezember 1808. Mit der Übernahme des französischen Verwaltungsmodells wurden auf den höheren Ebenen der administrativen Hierarchie Verwaltungskörperschaften eingeführt, die die von den alten Ständen bis dahin noch ausgeübten Funktionen bei der Steuerverteilung übernahmen ${ }^{56}$. Das politische Schicksal der altständischen Vertretungen war damit endgültig besiegelt. Am 24. März 1809 schieden sie auf Anordnung der Düsseldorfer Minister definitiv aus dem Verfassungsleben des Großherzogtums aus ${ }^{57}$.

Das Verschwinden der Stände aus dem konstitutionellen Gefüge erfolgte ohne großes Aufsehen. Reaktionen oder Proteste provozierte es nicht. Überhaupt blieb die von der Regierung oktroyierte Auflösung der Stände innenpolitisch ohne spürbare Konsequenzen, weil die Ständekollegien schon seit langem nicht mehr an den Staatsgeschäften teilgenommen hatten. Aus verfassungsrechtlicher Sicht markierte die Beseitigung der ständischen Kollegien hingegen eine Zäsur. Mit der Beseitigung des Dualismus im Verfassungsgefüge zog die Regierung die verfassungsrechtlichen Konsequenzen aus einer politischen Entwicklung, die bis weit in das 18. Jahrhundert zurückreichte. Sie führte Verfassungsnorm und Verfassungsrealität zusammen.

Das ersatzlose Ausscheiden der Stände aus dem Verfassungsleben war jedoch nicht endgültig. Obwohl es zunächst so aussah, als hielte Napoleon an der völligen Streichung der Stände fest, kam er im Winter 1811 auf das von Murat drei Jahre zuvor initiierte Reformvorhaben zurück und machte anläßlich seines Besuchs in Düsseldorf Anfang November 1811 erstmals politische Zugeständnisse für eine gesamtstaatliche Vertretungskörperschaft. Seine inhaltlichen Vorstellungen entsprachen dabei denen Murats. Er hatte vor, ein Organ zu schaffen, das in seinem Aussehen den westfälischen Ständen glich. Die Mitglieder dieses Repräsentativorgans sollten von künftig einzurichtenden Kantonsversammlungen gewählt werden und sich personell aus dem Kreis der Meistbesteuerten des Landes rekrutieren. Ihre Aufgaben entsprachen weitgehend denen der Generalräte der Departements. Sie hatten sich um die Steuervertei-

55 Noch kurz vor dem Herrscherwechsel Anfang Juli 1808 rief dieser ihm die Notwendigkeit zur Reformierung der altständischen Verfassung ins Gedächtnis, ibid.

56 Das galt auch für die Erben- und Kirchspieltage. Vgl. CAPPELle, Beiträge, S.66f.

57 Ministerieller Erlaß vom 24. März 1809, HStAD, GB, Nr. 47. 
lung zu kümmern, den Finanzminister zu kontrollieren und zu allem Stellung zu nehmen, was die Verwaltung betraf. Ferner mußten sie dem Landesherrn die Reklamationen der Untertanen überbringen. Darüber hinaus und abweichend von den westfälischen Verhältnissen gedachte Napoleon dem bergischen Staatsrat repräsentative Funktionen zu übertragen. Als eine Art Vertretungsorgan sollte dieser die Anliegen des Großherzogtums nach außen vertreten ${ }^{58}$.

Die wiederholt vorgetragenen Wünsche der Bevölkerung nach einer geschriebenen Verfassung waren nicht der einzige und wohl auch nicht der ausschlaggebende Grund für die Pläne zur Einführung eines Repräsentativorgans. Napoleons Reformabsichten hingen vielmehr eng mit den kurz vor dem Abschluß stehenden Planungen zur Einführung der französischen Gerichtsverfassung zusammen, denn die Laienrichter der künftigen Schwurgerichte hatten sich aus den Mitgliedern jener Körperschaften zu rekrutieren, welche die Delegierten der Repräsentativkörperschaft wählten: der Kantonsversammlungen. Um die personelle Besetzung der zu bildenden Geschworenengerichte zu ermöglichen, war es daher erforderlich, auch die bisher unbeantwortet gebliebene Frage der politischen Mitsprache nach französischem Beispiel zu regeln ${ }^{59}$.

Finanzminister Beugnot lehnte die Etablierung eines gesamtstaatlichen Repräsentativorgans entschieden $a b$ und schlug vor, statt dessen einen reduzierten Staatsrat einzurichten. Er meinte, dieser lasse sich wesentlich besser kontrollieren als eine Vertretungskörperschaft. Außerdem waren die bergischen Einwohner seiner Meinung nach noch nicht genügend von den Prinzipien durchdrungen, auf denen die französischen Institutionen beruhten. Insbesondere fehlte es ihnen nach Beugnot an der nötigen Bereitschaft, ehrenamtlich zu arbeiten. C'est supposer chez eux, meinte der kaiserliche Kommissar, des vertus qui ne sont encore que chez nous; et encore le noble préjugé de l'homme qui fait marcher tant de choses avant l'argent ${ }^{60}$. Institutionen, deren Mitglieder für ihre Tätigkeit kein Geld erhielten, waren für ihn daher im Großherzogtum von vornherein zum Scheitern verurteilt. Beugnot stützte sich hierbei auf Erfahrungen, die er während seiner Tätigkeit in Düsseldorf gemacht hatte. So hatten ihn zwei Vertreter des Ruhrdepartements am 9.Dezember 1811 darum gebeten, für einen Aufenthalt in Düsseldorf entschädigt zu werden. Das Großherzogtum, schloß Beugnot aus diesem Vorfall gegenüber Roederer Ende Dezember 1811, a besoin d'être gouverné quelque temps, sous l'influence non pas nominale mais très positive des loix françaises, avant qu'on puisse appeler avec quelque succès ses habitans à une représentation quelconque ${ }^{61}$. Wei-

58 Notes dictées par Sa Majesté vom 2. November 1811, AN, AF IV 1253; Protokoll der Sitzung Napoleons mit dem französischen Außenminister Maret, dem französischen Minister-Staatssekretär Daru sowie Roederer, Beugnot und Nesselrode vom 3. November 1811, AN, 29 AP 36.

59 François MonnIER, Justice, in: Tulard, Dictionnaire Napoléon, S. 993-999, hier S. 998.

60 Beugnot an Roederer in seinem Schreiben vom 26. Dezember 1811, AN, AF IV 1840.

61 Entschädigungsgesuch der Räte des Ruhrdepartements, Rumpe und Schniewind, vom 9. Dezember 1811, ibid. 
tere Argumente gegen die Errichtung eines Repräsentativorgans hatte der kaiserliche Kommissar bereits anläßlich der Planungen zur Umgestaltung der Exekutive im Herbst 1809 geäußert. Zum ersten hatte er in diesem Zusammenhang auf die mangelhafte Arrondierung des Großherzogtums und die noch ausstehende Konstitutionalisierung des Rheinbundes hingewiesen. Zum zweiten hatte er die politische Lage des Großherzogtums für nicht sicher genug gehalten, um ein solches Organ ins Leben zu rufen. Der Zweifel, in den die Zukunft des Großherzogtums gehüllt sei, führte er an, semble être une raison de ne rien précipiter à cet égard ${ }^{62}$. Zum dritten hatte er die Abhängigkeit des Großherzogtums von Frankreich gegen die Errichtung einer gesamtstaatlichen Vertretung angeführt. Da in Düsseldorf weder Gesetze diskutiert noch die Finanzen durch ein Vertretungsorgan geregelt werden mußten, war eine solche Körperschaft seiner Ansicht nach überflüssig. Selbst für die Repartition der Steuern bestand für ihn nicht die Notwendigkeit, ein Repräsentativorgan einzurichten. Die gleichmäßige Steuerverteilung hielt er durch die Arrondissement- und Departementräte, l'espèce de représentation $a u$ petit pié63 ${ }^{63}$ wie er diese nannte, für hinreichend gesichert. Diese seien um so wichtiger, erklärte er, qu'il n'y en aura point au dessus d'elles ${ }^{64}$. Die Einrichtung einer gesamtstaatlichen Repräsentation hielt er nur dann für sinnvoll, wenn das Großherzogtum Berg aus der politischen Abhängigkeit von Frankreich gelöst wurde ${ }^{65}$.

Beugnots Einwände gegen die Gründung einer Repräsentativkörperschaft blieben fruchtlos. Nur wenige Monate nach Verkündung seiner Reformabsichten setzte Napoleon sein Projekt in die Tat um, ohne die Vorbehalte seines Düsseldorfer Statthalters zu berücksichtigen. Am 15. März 1812 ordnete er an, im Großherzogtum ein neues gesamtstaatliches Vertretungsorgan einzurichten, und brachte damit die von Murat 1806 begonnene Reorganisation der Exekutive zum Abschluß ${ }^{66}$. Anders als im Königreich Westfalen, wo bei der Etablierung eines Repräsentativorgans die Namensgebung der historischen Vorläuferinstitutionen beibehalten wurde und die Versammlung unter der Bezeichnung „Stände des Reichs“ in den Verfassungstext einging, knüpfte man im Großherzogtum Berg nicht an derlei Traditionen in der Nomenklatur an, sondern bezeichnete das Repräsentativorgan als Kollegium. Grund dafür war vermutlich der andersartige Entstehungszusammenhang der Düsseldorfer Vertretung. Als diese etabliert wurde, waren die altständischen Körperschaften schon seit zwei Jahren aus dem politischen Leben geschieden. Das Kollegium ging deshalb nicht mehr, wie noch in Westfalen, unmittelbar aus den

62 Beugnot in seinem an Herzog Bassano gerichteten Mémoire sur l'organisation du gouvernement dans le Grand Duché vom 10. November 1809, AN, AF IV 642, plaq. 5099.

63 Ibid.

64 Ibid.

65 Ibid.

66 Décret impérial portant organisation du Conseil d'état et du Collège vom 15. März 1812, Gesetz-Bulletin, 2. Abtheilung, Nr. 93. 
Ständen hervor, sondern knüpfte an die nach französischem Vorbild modellierten Verwaltungsräte an.

Funktional übernahm das Kollegium die seit 1808 von den Departementund Arrondissementräten erledigten Pflichten und machte dadurch die höheren Verwaltungskörperschaften obsolet. Das neue Repräsentativorgan war für die Verteilung der direkten Steuern auf die einzelnen Administrationsbezirke zuständig, besaß gutachterliche Funktionen bei den Staatsfinanzen und konnte Wünsche und Verbesserungsvorschläge über Gesetze vorbringen. $\mathrm{Zu}$ diesem Zweck hatte sich das Kollegium auf Befehl des Großherzogs einmal jährlich $z u$ treffen. Ein Selbstversammlungsrecht besaß es nicht. Eine aus den Reihen der Mitglieder zu bildende fünfköpfige Deputation sollte dem Landesherrn die Wünsche und Ansichten des Kollegiums vortragen, nachdem dieses zusammengekommen war.

Wie die Verwaltungsräte bildete auch das neue Repräsentativorgan eine kollegiale Körperschaft. Es setzte sich aus insgesamt 85 Mitgliedern zusammen. Zehn davon ernannte der Landesherr aufgrund besonderer Verdienste. Die übrigen wurden von eigens dafür auf Kantonsebene zu bildenden Wahlmännerversammlungen aus der Liste der sechshundert Höchstbesteuerten des Großherzogtums bestimmt. Jeder Kanton mußte dabei eine ihm zugewiesene Quote in geheimer Abstimmung nach dem Prinzip der absoluten Mehrheit auswählen. Das Mandat war zeitlich unbegrenzt: Die Mitgliedschaft bestand lebenslänglich. Vakante Stellen waren alle fünf Jahre neu zu besetzen. Die Ersternennung der Wahlmänner, aus denen sich die Kantonsversammlungen der Notabeln zusammensetzten, behielt sich der Kaiser aus der Liste der Höchstbesteuerten der Kantone vor; anschließend sollten sich die Mitglieder in geheimer Abstimmung nach dem Mehrheitsprinzip kooptieren. Der kaiserliche Kommissar mußte sie hierzu versammeln. Wie die Mitglieder des Kollegiums sollten sich auch die Wahlmänner aus der Liste der Höchstbesteuerten rekrutieren, wobei Finanzminister Beugnot in einer Instruktion an die Präfekten vom 16. Juli 1812 entschied, daß bei der Erstellung der Vorschlagslisten für die Ernennung der Kantonsnotabeln etwa zur Hälfte auf Grundbesitzer zurückgegriffen werden sollte. Der Rest hatte aus Gewerbetreibenden und besonders qualifizierten Personen zu bestehen ${ }^{67}$. Auch die Mitglieder der Kantonsversammlungen wurden auf Lebenszeit aufgenommen ${ }^{68}$.

Zusammensetzung, Bestellung und Funktionen des Kollegums machen deutlich, daß es sich bei dem neuen Vertretungsorgan noch nicht wirklich um eine parlamentarische Körperschaft handelte. Zwar bestand das Kollegium aus Abgeordneten, die nicht nach geburtsständischen Kriterien ausgesucht, sondern gewählt wurden und auch nicht weisungsgebunden waren. Sie hatten die Aufgabe, das gesamtstaatliche Interesse zu vertreten. Doch war das aktive und passive Wahlrecht an den Besitz- und Vermögensstand der Untertanen

67 Schreiben Beugnots an die Präfekten vom 16. Juli 1812, HStAD, GB, Nr.144.

${ }^{68}$ Décret impérial portant organisation du Conseil d'état et du Collège, Art.7f. 
gebunden, so daß sich die Wählerschaft von vornherein auf einen kleinen Einwohnerkreis beschränkte und das Kollegium im Grunde ein Forum für die Angelegenheiten dieser Bevölkerungsgruppe darstellte. Da die Kantonsnotabeln, welche die Abgeordneten zu wählen hatten, sowie ein Teil der Kollegiumsmitglieder vom Souverän selbst ernannt wurden, fehlte es nicht nur den Wahlmännern, sondern ebenfalls den von ihnen gewählten Delegierten auf gesamtstaatlicher Ebene an Repräsentativität und demokratischer Legitimation. Allerdings wurde das landesherrliche Ernennungsrecht dadurch aufgeweicht, daß der Großherzog die Ernennungen auf Grundlage von Kandidatenlisten vornahm, die im vorhinein von den unteren Verwaltungsbeamten angefertigt und vom Finanzministerium bewilligt worden waren. Wie bei der Rekrutierung der übrigen Verwaltungsdiener lag die eigentliche Vorauswahl der Wahlmänner somit nicht in landesherrlicher Hand, sondern in der Verantwortung der zuständigen Administrativbehörden ${ }^{69}$. Ebensowenig besaß das Kollegium ein Selbstversammlungs- und Initiativrecht. Vielmehr konnte es nur durch den Landesherrn einberufen werden. Des weiteren übte es in Fragen der Gesetzgebung und der Finanzen nur bewilligende oder beratende Funktionen aus. Es bildete daher kein wirkliches parlamentarisches Kontrollorgan, sondern war in erster Linie eine herrschaftspolitisch motivierte Konzession des Monarchen.

Die eingeschränkten Funktionen des Kollegiums waren allerdings keine Besonderheit des Großherzogtums Berg, sondern ein allgemeines Kennzeichen der Verfassungsentwicklung in Deutschland. Des weiteren hatte sich im Königreich Westfalen gezeigt, daß es durchaus möglich war, im Rahmen der gesetzlich vorgegebenen Grenzen politisch Einfluß zu nehmen. Die westfälischen Stände hatten signalisiert, daß sie mehr darstellten als nur ein Akklamationsorgan der französischen Regierungspolitik ${ }^{70}$. Von daher scheint es tatsächlich nicht gerechtfertigt, im Hinblick auf das Kollegium »von Scheinkonstitutionalismus und -parlamentarismus zu sprechen ${ }^{71}$.

Die einheimische Bevölkerung nahm die von Napoleon veranlaßte Reformmaßnahme mit Euphorie auf. Offensichtlich sah sie in dem Kollegium eine Art Verfassungsersatz. Nesselrodes Aussagen zufolge erwartete das Volk die Aufstellung der Kandidatenlisten mit großer Ungeduld. Es fliege der Zeit voraus et voudrait déjà voir réaliser cette constitution, berichtete der Innenminister am 3. April 1812 seinem Vorgesetzten nach Paris ${ }^{72}$. Die Hoffnung der Untertanen auf den schnellen Vollzug der konstitutionellen Reform ging allerdings nicht in Erfüllung. Die Bildung des Repräsentativorgans verzögerte sich, weil, nicht anders als bei der Rekrutierung des lokalen Verwal-

69 Vgl. das Schreiben Beugnots vom 16. Juli 1812, HStAD, GB, Nr. 144.

70 OwZar, Verfassungspolitik, S.305, 307; vgl. ebenso HeCKER, Konstitutionalismus, S.152ff.

71 OWZar, Verfassungspolitik, S. 312.

72 Bericht Nesselrodes an Roederer vom 3. April 1812, AN, 29 AP 39. 
tungspersonals, die erforderlichen gesellschaftlichen Rahmenbedingungen fehlten, um die neuen Ämter ordnungsgemäß zu besetzen. Insbesondere standen nicht genügend Personen für die Zusammensetzung der Kantonsversammlungen zur Verfügung. Die mit der Kandidatensuche beauftragten Präfekten stießen bei der Anwendung der vorgeschriebenen Auswahlkriterien auf erhebliche Schwierigkeiten und mußten häufig Kompromißlösungen eingehen. Er habe unter den Höchstbesteuerten der Kantone nicht immer Personen ausfindig gemacht, die propres et capables aux fonctions du premier notable seien, klagte etwa der Präfekt des Siegdepartements gegenüber Beugnot Mitte August $1812^{73}$. Zwar habe er sich darum bemüht, die von Beugnot vorgegebenen Auswahlkriterien ${ }^{74} \mathrm{zu}$ befolgen, autant que les circonstances et localités l'ont permis. Doch sei er zu Konzessionen an die sozialen Verhältnisse vor Ort genötig worden. Die Bezeichnung des Notabeln, meinte er vor diesem Hintergrund, ne conviendra pas assez a [sic] tous ceux, qui ont été proposés ${ }^{75}$. Insgesamt dauerte es beinahe ein Jahr, ehe die Regierung die notwendigen Grundlagen für die Errichtung des Kollegiums geschaffen hatte. Am 9. Januar 1813 ernannte Napoleon die Mitglieder der für die Wahl der Abgeordneten zuständigen Kantonsversammlungen sowie die Präsidenten dieser Körperschaften. Gleichzeitig bewilligte er die Liste der Höchstbesteuerten des Großherzogtums, aus welcher sich das Kollegium rekrutieren sollte ${ }^{76}$.

Die Verfassungsreform gelangte über die vorbereitenden Maßnahmen nicht mehr hinaus, weil Roederer und Beugnot die dazu notwendige Einberufung der Notabelnversammlungen in den Kantonen immer wieder vertagten. Nach dem Ausbruch von Unruhen im Januar 1813 schoben sie die Zusammenkunft hinaus, um die Notabelnlisten von vermeintlich regierungsfeindlichen Personen zu säubern, wobei ihre Vermutungen von den Präfekten im nachhinein weitgehend widerlegt wurden. Aus den drei Departements wurde jeweils nur ein Fall bekannt, in dem einer der Kandidaten für die Liste der Kantonsnotabeln an den Aufständen teilgenommen hatte ${ }^{77}$.

Im September 1813 zögerten die beiden französischen Minister die Zusammenkunft der Kantonsnotabeln abermals hinaus, weil Beugnot die Einberufung der Wahlmänner aufgrund der außenpolitischen Krisenlage immer noch nicht für angebracht hielt.

73 Schreiben des Präfekten Schmitz an Beugnot 14. August 1812, HStAD, GB, Nr. 144.

74 La moralité et l'attachement au Gouvernement, hatte Beugnot den Präfekten am 16. Juli 1812 mitgeteilt, sont les principaux caractères qui devront dicter vos propositions. Schreiben Beugnots an den Präfekten vom 16. Juli 1812, ibid.

75 Schreiben des Präfekten Schmitz an Beugnot vom 14. August 1812, ibid.

76 Kaiserliches Ernennungsdekret vom 9. Januar 1813, AN, AF IV 711, plaq. 5696.

77 Schreiben Beugnots an Roederer vom 20. Juni 1813, ibid. plaq. 5697. Präfekt Spee hatte zudem beantragt, Arnold Frowein, den Maire von Wermelskirchen, wegen seines Verhaltens während der Unruhen in der Kandidatenliste durch eine andere Person zu ersetzen. Schreiben des Präfekten Spee an Beugnot vom 15. Juni 1813, HStAD, GB, Nr. 144. 
Le moment n'est assurément pas tel, warnte der kaiserliche Kommissar am 30. September 1813, qu'on puisse les appeler sans inquiétude à examiner les comptes généraux des recettes et dépenses de l'Etat, à exprimer des voeux sur les lois faites et à faire, et surtout à exercer une sorte de censure sur l'ensemble de l'administration. Personne ensuite ne peut répondre des événemens qui surviendraient durant la tenue des assemblées cantonnales ou celle du Collège, et rien n'est si imprudent que de réunir des hommes passionnés et de les laisser exposés au choc des événements imprévus. [...] J'estime donc, schloß er daraus, que c'est à la paix qu'il faut renvoyer la mise en activité du régime constitutionel dans le Grand-Duché. Alors ce régime qu'il serait dangereux d'essayer aujourd'hui, pourra commencer une ère nouvelle ${ }^{78}$.

Die beiden Minister steckten allerdings in einem Dilemma. Sie waren sich darüber im klaren, daß die Bevölkerung die Versammlung des Kollegiums erwarteten. Beugnot sollte deshalb die Ernennungsdekrete bereits an die Betroffenen ausgeben, um die Hoffnungen der Bevölkerung nicht zu enttäuschen - eine Hoffnung, die in Friedenszeiten sicherlich verwirklicht werde, meinte Roederer ${ }^{79}$.

Daß Roederer und Beugnot die Zusammenkunft der Kantonsnotabeln wiederholt hinausschoben und damit die Einrichtung des Kollegiums letztendlich vereitelten, war darauf zurückzuführen, daß sie Vertretungsorgane einerseits geringschätzten und diese möglichst auf Akklamationsfunktionen beschränken wollten ${ }^{80}$, Repräsentativkörperschaften aber andererseits als politischen Einflußfaktor grundsätzlich ernst nahmen. Sie erkannten, daß diese durchaus gewisse Handlungsspielräume besaßen und damit einen Unsicherheitsfaktor im politischen Alltag darstellten, unabhängig davon, wie gering ihre gesetzlichen Mitsprachemöglichkeiten waren. Sie sahen in diesen also mehr als nur ein scheinkonstitutionelles Element. Die Vorgänge in Westfalen gaben ihnen hierbei in gewisser Weise Recht. Für politisch zuverlässig hielten Beugnot und Roederer Vertretungsorgane nur in Zeiten innerer Stabilität. Deshalb verhinderten sie gerade im krisenhaften Jahr 1813, daß die Kantonsversammlungen zusammentraten bzw. das Kollegium etabliert wurde. Die Einberufung der Notabeln, machte Roederer gegenüber Beugnot am 24. September 1813 deutlich, bringe nur so lange keine Unannehmlichkeiten für die Regierung mit

78 So Beugnot in seinem Antwortschreiben an Roederer vom 30.September 1813, AN, AF IV 711, plaq. 5697.

79 Roederer in einer schriftlichen Anfrage an Beugnot vom 24. September 1813, ibid.

${ }^{80}$ In seinem Brief vom 31. August 1811 empfahl Roederer Beugnot, den Staatsrat bei allen Angelegenheiten hinzuzuziehen, ou il n'y a pas lieu d'appréhendre de mauvaises difficultés de la part du conseil d'etat [sic], denn mehrere Male habe Napoleon bei der Unterzeichnung von Gesetzen gefragt, ob der Rat diese billige. Vgl. HStAD, GB, Nr.127. Les conseils généraux, machte Roederer dem kaiserlichen Kommissar gegenüber in seinem Schreiben vom 23. September $1812 \mathrm{klar}$, als es um die Steuerverteilung für das Jahr 1813 ging, peuvent se plaindre des méthodes, des agens \&a. mais le ministre, après avoir fait de son mieux, doit toujours faire prévaloir son avis (HStAD, GB, Nr. 144). 
sich, que l'Esprit du pays serait conforme aux intérets du gouvernement, que les affaires générales paraissent en assez bonne situation pour ne laisser aucun doute sur la soumission des habitans, autrement la convocation du college [sic] $\&$ celle des notables serait un danger ${ }^{81}$.

\subsubsection{Mitsprachepraxis}

Die Bewertung der französischen Verfassungspolitik im Bereich der politischen Mitsprachemöglichkeiten der Einwohnerschaft steht nach wie vor in engem Zusammenhang mit der wissenschaftlichen Diskussion über Scheinkonstitutionalismus und Konstitutionalismus. Diese Auseinandersetzungen wurden lange Zeit von dem Blick auf die französischen Herrschaftsintentionen bestimmt. Neure Forschungen haben einen Weg aus dieser verengten Perspektive gewiesen und das Modernisierungspotential der von französischer Seite eingerichteten Vertretungsorgane in die Bewertung der napoleonischen Verfassungspolitik miteinbezogen. Sie kommen auf dieser Grundlage zu einer wesentlich positiveren Bilanz als bisherige Arbeiten ${ }^{82}$. Ein für die Beurteilung der verfassungspolitischen Maßnahmen Frankreichs zentraler Aspekt ist aber in den Forschungsdikussionen über die napoleonische Verfassungspolitik bis heute weitgehend unterbelichtet geblieben. Gemeint ist die Frage, welche Rolle die Einwohner im politischen Geschehen tatsächlich spielten, kurz: wie es um die politische Mitsprache faktisch bestellt war. Dieser Gesichtspunkt ist deshalb von Belang, weil die von der Regierung eingeräumten Partizipationsmöglichkeiten nur die vom Landesherrn eng gesteckten normativen Grenzen spiegeln. Über die tatsächliche Rolle, die die Bevölkerung im Verfassungsalltag spielte, geben sie wenig Aufschluß. Um die Frage nach dem Einfluß der Untertanen auf das politische Geschehen im Großherzogtum beantworten zu können, ist es notwendig, die Partizipationspraxis genauer in den Blick zu nehmen und die vorhandenen Spielräume und Chancen politischen Handelns innerhalb des von französischer Seite gesteckten konstitutionellen Rahmens zu ermitteln. Das soll im folgenden geschehen.

Partizipation wird in diesem Zusammenhang im weitesten Sinne verstanden als zweckorientiertes Handeln, um Entscheidungen auf den verschiedenen Ebenen des politischen Systems zu beeinflussen « 83 . Der terminologischen Offenheit liegen methodische Überlegungen zugrunde. Es sollen Formen und Inhalte der Partizipation herausgearbeitet werden, ohne durch eine allzu enge Definition Aktionsfelder von vornherein auszuklammern. Nur so ist es möglich, das gesamte Spektrum an Handlungsalternativen zu erfassen, das der Bevölkerung zur Verfügung stand, um politisch mitzuwirken. Deshalb wird neben

81 Roederer in einer schriftlichen Anfrage an Beugnot vom 24. September 1813, AN, AF IV 711, plaq. 5697.

82 Vgl. HeCKER, Konstitutionalismus; OwZAR, Verfassungspolitik.

83 Max KAASE, Partizipation, in: Dieter NoHLen (Hg.), Wörterbuch Staat und Politik, München, Zürich 1991, S.466-470, hier S. 466. 
der institutionell und konstitutionell gesicherten Teilnahme auch die funktionale, nicht vom Gesetz vorgesehene Partizipation in den Blick genommen.

\subsubsection{Legitime Partizipationsmöglichkeiten}

Institutionell gesicherte Teilnahmemöglichkeiten besaßen die Einwohner wegen des Nicht-Zustandekommens des Kollegiums nur auf regionaler und lokaler Ebene. Hierbei standen ihnen grundsätzlich zwei Formen der Partizipation zur Verfügung. Auf der einen Seite konnten sie seit Einführung der französischen Verwaltungsordnung im Oktober 1807 bzw. im Dezember 1808 in den Munizipalräten sowie in den Beratungsorganen auf Arrondissement- und Departementebene - hier aber nur bis 1812 - mitwirken. Nach dem Transfer der französischen Justizverfassung im November 1811 hatten die Einwohner auf der anderen Seite Gelegenheit, an der Rechtsprechung teilzunehmen, indem sie in einem der vier Assisenhöfe, die in den Verwaltungszentren der Departements errichtet wurden, das Amt eines Laienrichters übernahmen.

Die Schwurgerichte waren für jene Fälle zuständig, die vom Gesetz mit Zuchthaus, Verbannung, Pranger, Verlust der bürglichen Rechte oder schlimmstenfalls mit dem Tod bestraft wurden. Aufgabe der Laienrichter war es in diesem Rahmen, darüber zu urteilen, ob jemand eine strafbare Handlung begangen hatte oder nicht. Sie mußten entscheiden, machte der Präsident des Düsseldorfer Assisenhofes in seiner Eröffnungsrede am 6. Juli 1812 deutlich, inwiefern sie dieses oder jenes Mitglied unserer bürgerlichen Gesellschaft, noch ferner für werth halten, darin zu leben, oder [...] es für nöthig achten, daß dasselbe auf immer oder auf bestimmte Zeit daraus verstoßen werde ${ }^{84}$. Sie befanden somit über Leben und Tod der Angeklagten.

Die Zulassung zum Amt des Geschworenen war ähnlich reglementiert wie die Mitgliedschaft in den Ratskollegien und von vornherein nur einer kleinen Schicht der Bevölkerung vorbehalten. Laienrichter konnten nur diejenigen werden, die das Mindestalter von 30 Jahren erreicht hatten und zu den Höchstbesteuerten ihres Departements gehörten. Die Präfekten hatten die Aufgabe, eine Liste mit denjenigen Personen anzufertigen, die als Geschworene in Frage kamen. Aus dieser Urliste wählten die Präsidenten der Assisengerichte 36 Personen aus, von denen jeweils 12 zu Beginn der ersten Sitzung durch Losziehung als Geschworene ermittelt wurden. Wie die Ratsstellen wurde das Amt des Laienrichters ehrenamtlich bekleidet ${ }^{85}$.

Ergänzend zur institutionell gesicherten Partizipation auf Kommunal- bzw. Regionalebene verfügten die Einwohner über zwei nichtverfaßte, aber von

84 Eröffnungsrede des Präsidenten des Düsseldorfer Assisenhofs vom 6. Juli 1812, AN, AF IV 1834.

85 Walter NELLEN, Die Entwicklung des Düsseldorfer Gerichtswesens von der Einführung des Code Civil in Berg bis zum Inkrafttreten der Reichsjustizgesetze, in: Düsseldorfer Jahrbuch 50 (1960) S. 28-101, hier S. 51f.; zur Funktion und Bedeutung von Laienrichtern vgl. die Studie Petra Overaths, Tod und Gnade. Die Todesstrafe in Bayern im 19.Jahrhundert, Köln u. a. 2001, S.128f. 
der Regierung anerkannte und daher legitime Mittel zur Interessenartikulation. Zum einen konnten sie ihre Anliegen auf dem Petitionsweg an die Exekutive richten. Zum anderen stand es ihnen frei, mit Hilfe von Abgeordneten ihre Wünsche und Bitten höhernorts vorzustellen. Das Kollegium war, darauf ist bereits hingewiesen worden, gesetzlich sogar dazu verpflichtet, dem Kaiser regelmäßig seine Bemerkungen und Wünsche durch eine Deputation von fünf Mitgliedern vorzutragen ${ }^{86}$. Beide Formen der Interessenwahrnehmung hatten fest zum Repertoire der altständischen Gesellschaft gehört und waren deshalb tief in der politischen Kultur des Landes verankert. Beide konnten sowohl von den in den Ratsorganen vertretenen als auch von den dort nicht repräsentierten Einwohnern in Anspruch genommen werden. Allerdings bildeten Petitionen und die Entsendung von Deputierten für die offiziellen Vertretungskörperschaften nur zwei Instrumente von mehreren zur Durchsetzung ihrer Anliegen, während sie für die meisten Untertanen, die in den Räten gar nicht vertreten waren, die einzigen Möglichkeiten darstellten, ihre Interessen in legitimer Form zur Sprache zu bringen.

Während die Mitarbeit in den Verwaltungskollegien und den Schwurgerichten unter den Einwohnern des Großherzogtums nicht sehr begehrt war ${ }^{87}$ wobei hierfür die Gründe ausschlaggebend waren, die bereits in den Ausführungen über die Munizipalräte genannt worden sind -, machte die bergische Bevölkerung von der Möglichkeit zu supplizieren in hohem Maße Gebrauch. Petitionen erreichten die Exekutive in massenhafter Zahl aus allen Schichten der Bevölkerung. Es waren zumeist Einzelpersonen, die sich mit persönlichen Bitten an die Oberbehörden wandten. Mitunter setzten sich mehrere Personen bei der Regierung gemeinschaftlich für eine Sache ein. Dabei handelte es sich in der Regel um Mitglieder der neuen Verwaltungsräte, tradierte Verbände oder um Bündnisse, die aus einem gemeinsamen Interesse heraus kurzfristig geschlossen wurden. Nach der Reformierung des Düsseldorfer Magistrats im Herbst 1806 beispielsweise solidarisierten sich die Schöffen der Hauptstadt und richteten eine gemeinsame Eingabe an Nesselrode, um für den Verlust ihrer Magistratsstellen entschädigt zu werden ${ }^{88}$. Im Dezember 1810 baten 468 Weber des Siegdepartements den Innenminister in einer gemeinschaftlich verfaßten Supplik um wirtschaftliche Erleichterungen ${ }^{89}$.

Die Einwohner griffen ebenfalls auf das Institut der Deputation zurück. Sowohl die offiziell partizipierenden als auch die institutionell nicht vertretenen Bevölkerungskreise nahmen diese Möglichkeit zur Interessenartikulation für sich in Anspruch. Im Gegensatz zur schriftlichen Eingabe benutzten sie die

86 Gesetz-Bulletin, 2. Abtheilung, Nr. 36.

87 Vgl. hierzu etwa NeLLEN, Entwicklung, S.52f.

88 Vorstellung der Schöffen des Düsseldorfer Haupt- und Kriminalgerichts über den Verlust ihrer Nebenstellung und Erweiterung ihres Geschäftskreises vom 29. Dezember 1806, HStAD, GB, Nr. 4427.

89 Undatierte Petition der Weber des Siegener und des Netphener Kantons an den Innenminister (Dezember 1810), AN, 29 AP 54. 
Entsendung von Abordnungen jedoch nur als Ultima ratio. Abgeordnete verschickten sie erst, nachdem sie auf dem Petitionsweg nichts erreicht hatten. Deputationen kamen in französischer Zeit deshalb auch weitaus seltener vor als Gesuche. Auch hinsichtlich der damit verfolgten Motive unterschieden sich beide Artikulationsformen. Die in französischer Zeit organisierten Delegationen verstanden sich als Sachwalter der Bevölkerung. In dieser Funktion brachten sie ihre Unzufriedenheit mit bestehenden Gesetzen zum Ausdruck und klagten meist neues Recht ein bzw. stellten eminent politische Forderungen. Ansprüche auf die rechtliche Verankerung von Partizipationsrechten erhoben sie hierbei allerdings ebensowenig wie die Supplikanten. Bei der Mehrzahl der Bittschriften handelte es sich dagegen um Einzelanliegen mit dem Wunsch nach Ausnahmeregelungen. Gesuche mit einer auf das gesamte Großherzogtum gerichteten Zielsetzung waren eher selten. Das inhaltliche Spektrum der Eingaben war dabei weit gefächert. Es reichte von der Bitte um Einstellung bis zur Verwaltungsbeschwerde und umfaßte sowohl Anliegen, die auf das Ancien Régime verwiesen, als auch Wünsche proaktiver Natur. Bisweilen gingen Suppliken mit gegensätzlichen Forderungen ein. Gutes Beispiel dafür sind die Eingaben, die Bauern und Grundherren in Reaktion auf die Agrarreformen an die Regierung richteten ${ }^{90}$.

Der Grund für den unterschiedlich häufigen Gebrauch von Eingaben und Delegationen ist im besonderen Charakter der Deputation zu suchen. Anders als die Petition stellte die Abordnung schon per definitionem eine kollektive Angelegenheit dar. Ihre Mitglieder agierten im Auftrag einer Gruppe, deren Angehörige ihre Einzelwünsche zu einem gemeinsamen Willen zusammenfassen mußten. Deputationen verlangten von den Beteiligten deshalb ein hohes $\mathrm{MaB}$ an Zusammenhalt und Konsensbereitschaft, konnten aber auch weitaus mehr Repräsentanz beanspruchen als Suppliken. Hinzu kam, daß sich Abordnungen in der Regel immer noch direkt und ausschließlich an den Landesherrn wandten ${ }^{91}$, während schriftliche Eingaben mehr und mehr an die Verwaltungsbehörden adressiert wurden. Abordnungen erforderten somit wesentlich mehr Aufwand als Petitionen: Reise und Aufenthalt der Deputierten vor Ort mußten sowohl organisatorisch vorbereitet als auch finanziert werden, und dies war um so mühevoller und aufwendiger, als der Monarch zumeist außer Landes weilte.

Bei den Staatsdienern stieß der Rekurs der Bevölkerung auf die in der Frühen Neuzeit wurzelnden Formen der Interessenartikulation auf unterschiedli-

90 Vgl. u.a. folgende Eingaben: Petition der Münsteraner Grundbesitzer an Roederer vom Mai 1809 (mit einem Memorandum über die Natur der Leibeigenschaft in ihrer Gegend); Eingabe der ehemaligen Leibeigenen der vormaligen Grafschaft Tecklenburg an Beugnot vom 23. November 1810; undatierte Eingabe des Bauern Crane aus der Munizipalität Lütgendortmund (November 1810), AN, 29 AP 53; siehe ferner das Protokoll der Staatsratssitzung vom 3. Februar 1812, AN, AF IV 1837.

91 Eine Ausnahme bildete die von der Elberfelder Munizipalität an den Innenminister im Jahre 1808 gesandte und bereits näher erläuterte Deputation. Siehe Kap. III, 2.2.1.3. 
che Resonanz. Bittschriften wurden von den Amtsträgern in der Regel anerkannt und ernst genommen. Der hohe Aufwand, mit dem die in den Petitionen vorgebrachten Anliegen und Klagen behördenintern häufig untersucht und überprüft wurden, verdeutlicht dies ebenso wie die Tatsache, daß trotz wiederholter Drohungen selbst jene Eingaben bearbeitet wurden, die an den unteren Instanzen vorbeigeführt worden waren ${ }^{92}$.

Der Gebrauch von Deputationen fand bei den Staatsdienern dagegen weniger Beifall. Dabei war es nicht so sehr die Artikulationsform an sich, die sie ablehnten. Da sie wiederholt die Entsendung von Abgeordneten veranlaßten, hielten auch sie Deputationen offensichtlich für ein geeignetes Mittel, um politische Entscheidungen zu erwirken ${ }^{93}$. Was die Beamten offenbar störte, war die Eigenmächtigkeit, mit welcher die bergischen Untertanen ihre Vorhaben planten. Sie befürchteten wohl, daß der Landesherr auf diese Weise unmittelbare, möglicherweise unerwünschte Einblicke in Zu- bzw. Mißstände vor Ort erhielt, und es zu Kontrollverlusten oder ungewünschten Folgen vor Ort kam. Zwei Beispiele mögen das beleuchten.

(1) Im Frühjahr 1811 planten die ländlichen Gemeinden des Ruhr- und Emsdepartements, unter der Leitung des Bauern Giesbert Alef eine Delegation nach Paris zu entsenden, um dort vom Kaiser die Regelung der Leibeigenschaftsfrage zu erwirken ${ }^{94}$. Der Deputation vorausgegangen waren mehr als zwei Jahre andauernde Streitigkeiten zwischen Grundherren und Bauern

92 Vgl. die Ausführungen in Kap. III, 2.2.2.3.

93 Ende 1806 organisierte Finanzminister Agar auf Befehl Murats die Entsendung einer Delegation nach Warschau, um für das Großherzogtum wirtschaftliche Erleichterungen zu erwirken, und im Sommer 1813 schickte Innenminister Nesselrode eine zweiköpfige Deputation zum Kaiser nach Dresden, um den Aufschub des Verkaufs der durch das Dekret von Nossen konfiszierten Waren zu erbitten. Siehe das Schreiben Agars an den Dillenburger Provinzialrat Borcke vom 24. November 1806, HStAD, GB, Nr.34, das Schreiben Agars an Joachim Murat vom 11. Dezember 1806, ADD, Fonds Mosbourg, 100 MI 117, Nesselrodes Bericht an Roederer vom 28. Juni 1813 sowie die undatierte Depesche des Innenministers an den französischen Handelsminister Collin de Sussy, AN, 29 AP 34.

94 Schreiben des Innenministers an den Präfekten des Ruhrdepartements vom 24. Januar 1811, StAM, Nachlaß Giesbert von Romberg A, Nr. 166. Zur Genese bäuerlicher Interessenvertretungen: Werner Trossbach, Bauernprotest als "politisches Verhalten. Zu den Agrarkonflikten im Wetterau-Vogelsberg-Gebiet zwischen 1648 und 1806, in: Archiv für hessische Geschichte und Altertumskunde 42 (1984) S.73-124, besonders: S.104f.; Andreas WürGLER, Das Modernisierungspotential von Unruhen im 18. Jahrhundert. Ein Beitrag zur Entstehung der politischen Öffentlichkeit in Deutschland und der Schweiz, in: Geschichte und Gesellschaft 21 (1995) S.195-217, hier S. 209; Andreas SuTER, Regionale politische Kulturen von Protest und Widerstand im Spätmittelalter und in der Frühen Neuzeit, in: Geschichte und Gesellschaft 21 (1995) S.161-194, hier S.168; Jan Peters, Eigensinn und Widerstand im Alltag. Abwehrverhalten ostelbischer Bauern unter Refeudalisierungsdruck, in: Jahrbuch für Wirtschaftsgeschichte (1991/1992) S. 85-103, hier S. 98f.; Josef MooSER, Rebellion und Loyalität 1789-1848. Sozialstruktur, sozialer Protest und politisches Verhalten ländlicher Unterschichten im östlichen Westfalen, in: Peter STEINBaCH (Hg.), Probleme politischer Partizipation im ModernisierungsprozeB, Stuttgart 1982, S. 57-87, hier S. 66 . 
über die Frage, ob mit der Aufhebung der Leibeigenschaft auch die Dienste und Abgaben entschädigungslos abgeschafft waren. Während sich die Mehrheit der abgaben- und dienstpflichtigen Bauern als gänzlich befreit betrachtete und demzufolge die Leistung der Dienste und Abgaben einstellte, behaupteten die Grundherren das Gegenteil. Die Rechtsprechung, die die Konfliktbeilegung zunächst zu regeln hatte, brachte keine klare Entscheidung. In erster Instanz wurden häufig die Bauern freigesprochen. Die höheren Tribunale gaben demgegenüber meist den Grundherren Recht ${ }^{95}$. Als sich abzeichnete, $\mathrm{da} B$ auf dem Gerichtsweg nichts zu erreichen war, entschlossen sich die Bauern, ihr Anliegen durch eine aus ihren Reihen gebildete Delegation dem Kaiser direkt vorzutragen. Ihre Beschwerden richteten sich hierbei primär dagegen, daß die Grundherren die Abgaben und Dienste weiterhin einforderten. In zweiter Linie griffen sie jedoch die Justiz und damit den Staat selbst an. Vor allem dadurch gewannen ihre Ansprüche an politischer Sprengkraft. Finanziert wurde die Reise nach Paris durch Geldsammlungen in den beteiligten Gemeinden ${ }^{96}$. Fachlichen Beistand leistete unter anderem Arnold Mallinckrodt, der als Präfekturrat, Advokat und Zeitungsherausgeber zugleich tätig war $^{97}$. Als Beamter half Mallinckrodt den Bauern mit seinem politischen Know-how, als Advokat stellte er ihnen ein Beratungsbüro zur Verfügung, als Zeitungsherausgeber sorgte er dafür, daß das bäuerliche Anliegen an die Öffentlichkeit gelangte ${ }^{98}$.

Bei den Behörden stieß das Unternehmen der Bauern auf Kritik und Widerstand. Präfekt Romberg hielt die Forderungen der Bauern für unrechtmäBig und nachteilig für die Grundbesitzer ${ }^{99}$. Der Kommunalleiter der Mairie Vornholz befürchtete, daß alle Gutsherren mit jedem Bauer im Proces kommen, und daß hierdurch ein außerordentliches Geldquantum von gewinnsüchtigen Menschen erpresset werde, dabei Mißtrauen und Widersetzlichkeiten bewurekt werden ${ }^{100}$. Beugnot sah in dem Projekt ein Zeichen innerer Unruhe

95 Vgl. etwa die undatierte Eingabe des Bauern Crane aus der Mairie Lütgendortmund (November 1810), AN, 29 AP 53, die Supplik des Regierungs- und Präfekturrats Arnold Mallinckrodt an Napoleon vom 22. März 1813, AN, AF IV 1837, sowie die Schreiben Arnold Mallinckrodts an Roederer vom 8. und 28. April 1813, ibid.

96 Schreiben des Innenministers an den Präfekten des Ruhrdepartements vom 24. Januar 1811; Bericht des Vornholzer Maires Harrier an den Unterpräfekten Wiethaus vom 21.Februar 1811; Schreiben des Maires von Werne, Schlebrügge, an den Präfekten Romberg vom 4. Februar 1811, StAM, Nachlaß Giesbert von Romberg A, Nr. 166.

97 Zur Biographie Mallinckrodts vgl. Gustav Lunrowski, Arnold Mallinckrodt, in: Westfälische Lebensbilder 15 (1990) S. 91-107; Kurzweg, Presse, S.347.

98 Bericht Nesselrodes an Roederer vom 3. April 1812, AN, 29 AP 39. Am 30. Dezember 1810 ließ Mallinckrodt die von ihm verfaßte und der Deputation nach Paris mitgegebene Schrift Die Belehrung des Bauernstandes« im Westfälischen Anzeiger abdrucken. Vgl. AN, 29 AP 53.

99 Schreiben des Präfekten Romberg an den Innenminister vom 6. Februar 1811, StAM, Nachlaß Giesbert von Romberg A, Nr. 166.

100 Bericht des Vornholzer Maires Harrier an den Unterpräfekten Wiethaus vom 21. Februar 1811, ibid. 
und in Alef einen agent d'intrigue ${ }^{101}$. Die Bauern des Ruhrdepartements, teilte er Roederer mit, s'assemblent et s'excitent à la résistance. Ces dispositions leur sont naturelles. Ils etaient signalés fort remuans sous le Gouvernement Prussien et envoyaient aussi des Députés à Berlin ${ }^{102}$. Generalprokurator Sethe, der sich ansonsten für die Belange der einheimischen Bevölkerung einsetzte, hieß die Deputation ebenfalls nicht gut. Zum einen glaubte er nicht, daß die Rechtsprechung die Interessen der Grundherren vertrat ${ }^{103}$. Zum anderen war er in der Ablösungsfrage anderer Auffassung als die Bauern. Er trat sogar öffentlich als entschiedener Gegner der bäuerlichen Forderungen auf, wie das Gefecht über die Behandlung der märkischen und bergischen Zeitgewinnsgüter verdeutlicht, das er mit Mallinckrodt führte ${ }^{104}$.

(2) Im Frühjahr 1811 beauftragte die bergische Kaufmannschaft mehrere Vertreter aus ihren Reihen damit, bei Napoleon die Vereinigung des Großherzogtums mit dem französischen Kaiserreich persönlich zu erbitten ${ }^{105}$. Anlaß zu der Deputation gab die Annexion Hollands durch Frankreich Ende 1810. Die tieferen Ursachen sind dagegen in den verheerenden Folgen zu suchen, die durch die immer schärfer werdenden Schutzzollbestimmungen Napoleons im Wirtschaftskampf gegen England hervorgerufen wurden. Die ökonomischen Prohibitionsmaßnahmen blockierten die Handelsbeziehungen Bergs mit Übersee und schnitten die bergischen Waren von lebensnotwendigen Märkten wie dem Italiens ab. Geschützt vor der englischen Konkurrenz und begünstigt durch die Erfordernisse des Krieges hatten zwar einzelne Wirtschaftszweige wie die Baumwoll- und Klingenindustrie von der französischen Zollpolitik profitieren können. Auch die Kohleindustrie hatte daraus Nutzen gezogen, weil die englische Konkurrenz ferngehalten wurde und die Kohlepreise anstiegen ${ }^{106}$. Die meisten Industrie- und Gewerbezweige litten dagegen erheblich unter den Folgen der Kontinentalsperre. Sie verloren nicht nur wichtige Absatzgebiete, sondern wurden zudem von notwendigen Rohstoffquellen abgeschnitten. Massenentlassungen, Arbeitslosigkeit und Auswanderungen waren die Folge. Die Verarmung der Bevölkerung nahm drastisch zu und mit ihr die Kriminalität ${ }^{107}$.

101 Beugnot in seinem Schreiben an Roederer vom 18. August 1813, AN, 29 AP 53.

102 Ibid.

103 Undatierter Bericht des Generalprokurators Sethe an Beugnot, ibid.

$104 \mathrm{Vgl}$. Christoph Wilhelm Henrich SETHE, Urkundliche Entwicklung der Natur der Leibgewinngüter und Widerlegung der von dem Herrn Regierungsrath Mallinkrodt darüber im Westphälischen Anzeiger vorgetragenen irrigen Behauptungen, Düsseldorf 1810.

105 Schreiben Nesselrodes an Roederer vom 4. März 1811, AN, 29 AP 39.

106 Reisetagebuch des kaiserlichen Kommissars, S. 186; Louis BERGERon, Remarques sur les conditions du développement industriel en Europe occidentale à l'époque napoléonienne, in: Francia 1 (1973) S.537-556, hier S.547f.

107 Nach Schätzungen Roederers ging die Zahl der im Großherzogtum Beschäftigten zwischen 1807 und 1811 von 57600 auf 38681 zurück. Siehe den Bericht Roederers an Napoleon (Dezember 1810), AN, 29 AP 54, ferner den Polizeibericht der Mairie Hilden des Monats Juni 1811, in: Heinrich StrangmeIER (Hg.), Hildener und Benrather Polizeiberichte aus der Franzosenzeit, in: Hildener Jahrbuch (1965-1970) S.115-188, hier S.134, sowie das Reisetagebuch des kaiserlichen Kommissars, S.187. 
Schon früh hatten Kaufleute und Industrielle die Regierung darum gebeten, die Zölle zu senken. Ihr Anliegen war aber nur auf wenig Gehör gestoßen. Zwar autorisierte Napoleon zu Beginn des Jahres 1807 den Handel dazu, bergische Stoffe in begrenztem Umfang nach Italien zu liefern. Doch hatte diese Verfügung nur vorübergehend Bestand. Bereits im Dezember desselben Jahres hob der Kaiser die Einfuhrerlaubnis wieder auf ${ }^{108}$. Einige Unternehmer versuchten dem wirtschaftlichen Ruin durch die Verlagerung ihrer Betriebe auf das linksrheinische Ufer zu entgehen - eine Maßnahme, die im ehemaligen Herzogtum Berg bereits in revolutionärer Zeit üblich gewesen war ${ }^{109}$. Die Ansiedelung industrieller Zweigstellen links des Rheins bot sich jedoch nicht für alle Betriebszweige an. Gerade die Betriebe des Metallgewerbes waren standortgebunden und konnten nicht verlagert werden.

Als Napoleon im Sommer 1810 den Tarif auf Kolonialwaren verschärfte, spitzte sich die wirtschaftliche Lage im Großherzogtum derart $\mathrm{zu}^{110}$, daß viele Gewerbetreibende den einzigen Ausweg aus der wirtschaftlichen Misere darin sahen, das Großherzogtum dem Empire zu inkorporieren und es damit politisch und wirtschaftlich Frankreich einzugliedern ${ }^{111}$. Bei den Verwaltungsbehörden gingen zahlreiche Petitionen mit dem Wunsch um Vereinigung ein ${ }^{112}$. Le mot sreunion avec la France, schrieben Remscheider Kaufleute, serait le mot, qui nous rendrait la vie ${ }^{113}$. Offenbar war ihnen weniger an der staatlichen Existenz gelegen als am Genuß der wirtschaftlichen Vergünstigungen des französischen Kaiserreichs. Die Versuche der Kaufmannschaft, die Annexion auf dem Weg der Bittschrift zu erreichen, blieben allerdings fruchtlos. Als das Großherzogtum schließlich durch die Einverleibung Hollands in das Empire Ende 1810 auch noch den Zugang zum Meer verlor, traten einige Handelstreibende die Flucht nach vorn an und entschieden sich dazu, Abgeordnete nach Paris zu schicken, um der französischen Regierung die Bitte um Vereinigung persönlich vorzutragen.

108 Schmidt, Grand-Duché, S. 343; RedLich, Anwesenheit, S. 192.

109 RingEL, Wirtschaft, S. 160.

110 Reisetagebuch des kaiserlichen Kommissars, in: Bergische Forschungen 15 (1978) S.165-161, hier S.187; ScHmidT, Grand-Duché, S. 354f.; Reulecke, Wirtschaft, S. 25.

111 Aufgrund der zu erwartenden Schwierigkeiten in Industrie und Handel beantragte Innenminister Nesselrode in seinem Schreiben an den Kaiser vom 21. September 1810, arbeitslose Untertanen im Straßenbau einzusetzen, HStAD, GB, Nr.4211.

112 In Remscheid und Barmen baten die Munizipalbeamten darum, in Lennep, Hilden und Mülheim an der Ruhr wurde der Wunsch aus den Reihen der Fabrikanten und Arbeiter vorgetragen. In Elberfeld taten sich die Bediensteten der Munzipalität und Vertreter des Handels zusammen. Vgl, die undatierte Eingabe des Remscheider Maires Diederichs an Napoleon, die Eingabe des provisorischen Maires Barmens an Roederer vom 16. November 1810, das Gesuch der Lenneper Leinenhersteller vom 9. November 1810, die Supplik der Hildener Fabrikanten an Napoleon vom 26. Januar 1811, das Gesuch der Mülheimer Bergbauarbeiter an den Kaiser vom 25. Februar 1811, die Eingabe der Elberfelder Beigeordneten, des Kaufmanns Siebel und des Handelsvorstehers Johann Kaspar Nieland an Napoleon vom 31.Januar 1811, AN, AF IV 1839A.

113 Zit. nach Justus Hashagen u. a. (Hg.), Bergische Geschichte, Düsseldorf 1938, S. 241. Vgl. auch ScHMIDT, Grand-Duché, S.383. 
Angeregt und in die Wege geleitet wurde die Delegation von den Handelsvorständen Barmens und Elberfelds. Mit der Behauptung, die Vereinigung Bergs mit Frankreich sei das alleinige Rettungsmittel des Großherzogtums und werde vom gesamten Handlungs- und Manufakturstand gewünscht, arbeiteten die Barmer und Elberfelder Wirtschaftsbürger seit Februar 1811 darauf hin, möglichst viele Einwohner für ihr Vorhaben zu gewinnen ${ }^{114}$. Die Abordnung, die auf ihre Initiative hin zustande kam und damit in gewisser Weise eine Fortsetzung der Interessenvertretung des Wirtschaftsbürgertums aus vorfranzösischer Zeit war ${ }^{115}$, setzte sich aus jeweils einem Ratsmitglied der Munizipalitäten Barmen und Elberfeld sowie aus einem Mitglied der Generaldepartementräte von Rhein und Sieg zusammen. Die Mehrzahl der Deputierten entstammte mithin dem ehemaligen Herzogtum Berg. Das Ruhrdepartement entsandte keinen eigenen Abgeordneten in die französische Hauptstadt, sondern ließ sich durch den Vertreter des Rheindepartements repräsentieren ${ }^{116}$.

Die Kaufmannschaft fand bei vielen Beamten keine Unterstützung für ihr Vorhaben. Der Präfekt des Rheindepartements lehnte das Unternehmen ab, weil er es für vermessen hielt, daß sich die vierköpfige Delegation als Repräsentativorgan der großherzoglich-bergischen Bevölkerung verstand. Kein Gesetz gebe ihr das Recht dazu ${ }^{117}$. Innenminister Nesselrode sprach sich aus denselben Gründen wie Präfekt Borcke gegen das Vorhaben der Handelstreibenden aus. Auch für ihn durften sich die Wirtschaftsbürger nicht zu Fürsprechern des Großherzogtums machen, sondern sie mußten sich darauf beschränken, ihre konkreten Interessen vorzutragen ${ }^{118}$. In seinen Augen war einzig die geplante Repräsentativkörperschaft dazu legitimiert, Ansprüche im Namen der bergischen Einwohnerschaft zu erheben ${ }^{119}$. Nur sie repräsentierte für ihn die gesamte Öffentlichkeit. Darüber hinaus lehnte Nesselrode das Unternehmen der Kaufmannschaft auch aus Angst vor dem Verlust der Eigenstaatlichkeit des Großherzogtums ab. Er befürchtete, daß sich die Lage des Großherzogtums durch die Vereinigung mit Frankreich nicht verbesserte, sondern im Gegenteil verschlechterte. C'est même, peut être, meinte er in Bezug auf die Deputation der Kaufmannschaft, tout ce qu'ils pourroint se [sic] flatter d'obtenir ${ }^{120}$.

114 Schreiben des Barmer und Elberfelder Handelsvorstands an den Maire der Munizipalität Wipperfürth vom 1. Februar 1811, HStAD, GB, Nr. 9688.

115 ENGELBRECHT, Herzogtum, S. $245 f$.

$116 \mathrm{Er}$ wurde am 17. April 1811 von einigen Munizipalitäten des Ruhrdepartements mit dieser Aufgabe betraut. Vgl. AN, AF IV 1839A.

117 Schreiben Borckes an den Unterpräfekten von Elberfeld vom 13. Februar 1811, HStAD, GB, Nr. 189.

118 Man habe den Abgeordneten derart zugeredet, daß sie der Auffassung seien, qu'ils peuvent se gerer deputes du pays [sic], berichtete er Minister-Staatssekretär Roederer in seinem Schreiben vom 31. März 1811, AN, 29 AP 39.

119 Ibid.

120 Ibid. Noch im September 1813 hatte Nesselrode Angst vor dem Verlust der staatlichen Souveränität. Bricht das Mindeste bey uns aus, schrieb er Präfekt Romberg in seinem 
Wiewohl die höheren Verwaltungskräfte den organisierten Zusammenschluß der Bevölkerung oftmals mißbilligten, besaßen sie keine rechtliche Handhabe, um die Entsendung von Deputationen zu verhindern. Sie durften den Einwohnern den Zutritt zum Thron nicht verbieten, und konnten allenfalls versuchen, die Pläne der Untertanen auf Umwegen zu konterkarieren. Präfekt Romberg etwa wollte die geplante Deputation der Bauern zu Fall bringen, indem er dem Bauern Alef die Ausstellung des für die Reise nach Paris notwendigen Passes verweigern ließ, weil dieser im Jahre 1809 an einem Aufruhr beteiligt und deswegen zu neun Monaten Haft verurteilt worden war ${ }^{121}$. Rombergs Bemühungen scheiterten jedoch. Es gelang Alef, als Reitbursche eines Händlers in die französische Hauptstadt zu reisen ${ }^{122}$.

Ohnehin lehnten nicht alle Staatsdiener die Vorhaben der Einwohner ab. Im Gegenteil gab es Beamte, die die Abordnungen befürworteten oder unterstützten. Beispielsweise wurde der Wunsch der Bauern des Ruhr- und Emsdepartements nach einer endgültigen Regelung der Dienste und Abgaben von Mitgliedern der kommunalen Verwaltung durchaus gutgeheißen. Einige Gemeindediener beteiligten sich sogar aktiv an den Reisevorbereitungen des Bauern Alef, indem sie organisatorische Aufgaben wie etwa die Sammlung von Geld für den Aufenthalt des Deputierten vor Ort übernahmen ${ }^{123}$. Die von der Kaufmannschaft organisierte vierköpfige Delegation erhielt von Beugnot Rückendeckung. Wie die Kaufleute war es für ihn unmöglich, que le Grand Duché subsiste au sein de la France qui le presse de toutes parts, qui lui apporte tant qu'elle veut, tout ce qu'elle veut, et qui refuse d'en rien reçevoir, und daß es deswegen notwendig sei, das Großherzogtum dem Empire zu inkorporieren ${ }^{124}$. Außerdem hielt er im Unterschied zu seinem Kollegen im Innenministerium die persönlichen Anliegen der Handel- und Gewerbetreibenden mit dem gesamtstaatlichen Interesse sehr wohl für kompatibel. [...] le voeu $d u$ commerce, notierte er am 31.März 1811, est ici trop en harmonie avec l'esprit général du Grand-Duché, pour que je me refuse à y prêter [...] mon adhésion $^{125}$. Beugnots energisches Eintreten für die Annexion war wohl zudem darauf zurückzuführen, daß er in dieser Zeit auf seine Versetzung nach Frank-

Schreiben vom 3. September 1813, so ist nichts gewißer als daß das ganze Großherzogthum im Kriegsstand erklärt wird, und irgend einen Gouverneur militaire bekommt, wie es mit den hanseatischen Departements der Fall war. StAM, Nachlaß Giesbert von Romberg A, Nr. 8.

121 Bericht Beugnots an Roederer vom 25. August 1811, AN, 29 AP 53. Zunächst hatte er sogar geplant, die Gelder und Papiere der Organisatoren konfiszieren zu lassen. Schreiben Rombergs an Wiethaus vom 5. Februar 1811, StAM, Nachlaß Giesbert von Romberg A, Nr. 166.

122 Bericht Beugnots an Roederer vom 25. August 1811, AN, 29 AP 53.

123 Schreiben des Vornholzer Maires an den Unterpräfekten vom 21. Februar 1811, StAM, NachlaB Giesbert von Romberg A, Nr. 166.

124 Schreiben Beugnots an Roederer vom 31. März 1811, AN, AF IV 1839A.

125 Notiz Beugnots in der Vorschrift eines Schreibens an den Präfekten Borcke vom 16. Februar 1811, HStAD, GB, Nr.189. 
reich hinarbeitete ${ }^{126}$ und in Paris im übrigen bereits selbst um die Vereinigung des Großherzogtums mit Frankreich gebeten hatte ${ }^{127}$.

Mit Ausnahme der Laienrichter, deren Entscheidungen Rechtskraft besaBen und von der Regierung nur schwer rückgängig gemacht werden konnten, waren die Aussichten der Einwohner auf die erfolgreiche Durchsetzung ihrer Interessen gering. Weder Petenten noch Delegierte besaßen politische Druckoder weiterführende Rechtsmittel, um ihren Angelegenheiten Nachdruck und Gewicht zu verleihen. Ob ihren Wünschen und Bitten stattgegeben wurde, hing somit weitgehend von den Oberbehörden und in letzter Instanz vom Landesherrn selbst ab. Gleiches galt im Grunde für die Verwaltungsräte. Da sie keine Entscheidungskompetenzen, sondern ausschließlich Beratungsfunktion hatten, waren sie ebenfalls darauf angewiesen, daß ihre Beschlüsse von der Regierung und vor allem von der Düsseldorfer Ministerialbürokratie gebilligt wurden.

Nicht alle Einwohner wollten allerdings die Verwirklichung ihrer Interessen allein dem Wohlwollen der Regierung überlassen. Während es, wie bereits erläutert, immer wieder vorkam, daß Supplikanten zur Verbesserung ihrer Chancen bewußt den von der Verwaltungsordnung vorgeschriebenen Instanzenzug umgingen und sich direkt an die Oberbehörden wandten oder sich von Beamten oder Honoratioren protegieren ließen, bemühten sich die Organisatoren von Deputationen darum, möglichst viele Einwohner für ihr Vorhaben zu gewinnen. Um die Erfolgsaussichten ihres Unternehmens zu fördern, setzten sie außerdem gleichzeitig Petitionen ein, zumal die Gesuche auf diese Weise direkt in die Hände der Regierungsbehörden oder des Landesherrn gelangten. Beispielsweise trug die 1811 nach Paris gesandte Abordnung aus den Reihen der Bauern des Ruhrdepartements eine schriftliche Vorstellung ihres Antrags bei sich ${ }^{128}$. Darüber hinaus überbrachte sie dem Kaiser eine Bittschrift der Unnaer Müller, in welcher diese die Aufhebung des Mühlenzwangs wünschten ${ }^{129}$. Die Deputation der Händler, die sich 1811 nach Paris begab, um dort die Annexion des Großherzogtums durch Frankreich zu erbitten, hatte einen Brief Beugnots bei sich, in dem dieser ihr Ansuchen unterstützte.

Verwaltungsräte, Supplikanten und Deputierte blieben jedoch grundsätzlich vom Entgegenkommen der Regierung und in letzter Instanz von den Herrschaftsinteressen Frankreichs abhängig. Am deutlichsten spürten dies wohl die Abgeordneten der Kaufmannschaft im Frühjahr 1811. Obwohl sie von Beugnot unterstützt wurden und obwohl sie beteuerten, nicht nur von den wirtschaftlichen Vorteilen Frankreichs profitieren, sondern auch die öffentli-

126 Vgl. Kap. III, 1.2.

127 Schreiben Beugnots an den französischen Polizeiminister Savary vom 15. Februar 1813, AN, F/7/6549.

${ }_{128}$ Undatierte Petition des Bauern Alef an Napoleon (1811), AN, 29 AP 53.

129 Eingabe der Müller Opherdickes vom 5. Mai 1811, ibid. 
chen Lasten mittragen zu wollen ${ }^{130}$, mußten sie unverrichteter Dinge in das Großherzogtum zurückkehren. Sie hatten keine Audienz bei Napoleon erhalten. Ihre Bitte um Vereinigung wurde ebensowenig erfüllt, weil sie sich nicht mit den Wirtschaftsinteressen Frankreichs vereinbaren ließ, die für die französische Regierung stets Vorrang vor den bergischen Angelegenheiten besaBen $^{131}$.

Dennoch erreichten bergische Einwohner im Rahmen ihrer begrenzten Handlungsmöglichkeiten mitunter ihr angestrebtes Ziel. Die Ausführungen zur Kommunalreform haben beispielhaft deutlich gemacht, wie es mehreren Gemeinderäten gelang, ihre Interessen höhernorts durchzusetzen. Als vorteilhaft erwies sich für die Räte hierbei die Umbruchsituation, die durch den Transfer des französischen Verwaltungsmodells entstand. Beim Übergang vom altständischen Verfassungsgefüge zum französischen Modell ergaben sich Freiräume, die politisches Handeln möglich machten. Politische Bedeutung erlangten auch die Laienrichter, als es darum ging, die während der Proteste zu Beginn des Jahres 1813 festgenommenen Personen zu verurteilen. Unter dem Vorwand, die Aufständischen hätten unter Zwang gehandelt, erklärten sie diese im nachhinein oftmals für unschuldig und brachten dadurch ihre Rechtsauffassung im Strafverfahren ein. Die Regierung kitisierte die milde Spruchpraxis der Geschworenen zwar, griff aber nicht in die Schwurgerichtsbarkeit selbst ein, sondern umging die Assisenhöfe, indem sie vermehrt Militärkommissionen einsetzte, um Aufständische verurteilen zu lassen ${ }^{132}$.

Ebenso war es möglich, auf nichtverfaßtem Weg zu reüssieren. Beispiele, in denen die Wünsche von Petenten erfüllt wurden, sind nicht selten. Auch Deputationen führten gelegentlich zu positiven Ergebnissen. Der Fall Elberfeld ist bekannt. Erfolgreich war auch der Bauer Alef, denn er veranlaßte mit seiner Vorstellung den französischen Kaiser zumindest dazu, dem Anliegen der Bauern nachzugehen. Philippe-Antoine Merlin de Douai, der Generalprokurator des Pariser Kassationshofs, sowie ein weiteres Mitglied dieses Gerichts wurden von Napoleon damit beauftragt zu prüfen, si les Paysans $d u$ Grand Duché de Berg sont fondés dans leurs plaintes ${ }^{133}$. Ergebnis dieser erneuten Verhandlungen war eine Kompromißlösung: das Ablösungsdekret vom 13. September 1811, das einen Ausgleich zwischen grundherrlichen und bäuerlichen Interessen schaffen sollte ${ }^{134}$. Für Alef persönlich hatte das Un-

130 Mais ces charges, ces contributions, ces vicissitudes, erklärten die Deputierten am 3.Mai 1811 Roederer gegenüber mit Nachdruck, qui pèsent sur la France, nous voulons y participer aussi. Nous voulons les supporter aussi. Nous voulons devenir français (AN, AF IV 1839A).

131 Vgl. das Schreiben Roederers an Nesselrode vom 10. März 1811, AN, 29 AP 39.

132 Vgl. Kap. IV, 2.2.2.

133 Randnotiz Napoleons vom 9.Juli 1811 auf der Petitionsschrift des Bauern Alef, AN, 29 AP 53.

134 Gesetz-Bulletin, 2. Abtheilung, Nr. 43. Die Bauern wurden durch den Erfolg ihres Unternehmens so ermutigt, daß sie nach Erlaß des Septemberdekrets erneut eine Delegation nach Paris schickten. Diese solite um die vollständige Ausführung des neuen Gesetzes bitten. Eingabe der Deputierten vom 12. Oktober 1811, AN, 29 AP 53. 
ternehmen indes ein Nachspiel. Da die Unterbehörden in ihm sowohl den Spiritus rector der Angelegenheit als auch einen potentiellen Aufrührer sahen, stellten sie ihn unter Überwachung und untersagten ihm, seinen Wohnort ohne die Aufsicht des Maires zu verlassen ${ }^{135}$.

\subsubsection{Protest als Ultima ratio}

Abgesehen davon, daß die Bevölkerung von der institutionell und konstitutionell gesicherten Teilnahme vielfach Gebrauch machte, um zu partizipieren, waren in großherzoglich-bergischer Zeit die Übergänge zwischen legalen und illegalen Handlungsformen fließend. So stellten gerade Formen des passiven Widerstandes gegen geltende Gesetze, wozu insbesondere Verstöße gegen die Steuer-, Zoll- und Konskriptionsgesetze zählten, keine Seltenheit dar und eskalierten in dem Maße, wie sich die innenpolitische Situation, bedingt durch die verschärfte Kontinental- und Militärpolitik Napoleons, verschlechterte ${ }^{136}$. Im Januar 1813 schlug der passive Widerstand schließlich in offene Proteste um $^{137}$.

Anstoß dazu gaben Nachrichten von den Verlusten der Grande Armée in Rußland, die das Großherzogtum trotz der Zensurmaßnahmen von französischer Seite erreichten. Durch sie wurden Gerüchte über die nahende Ankunft russischer Truppen in Umlauf gebracht ${ }^{138}$. Hinzu kam, daß sich unter den Gefallenen auch bergische Soldaten befanden. Eigentlicher Auslöser der Unruhen waren aber erneute Truppenaushebungen für den französischen Rußlandfeldzug. In den Kantonen Düsseldorf, Ratingen, Elberfeld und Barmen lief die

135 Schreiben Arnold Mallinckrodts an Roederer vom 28. April 1813, AN, AF IV 1837.

136 Vgl. Kandil, Sozialer Protest, passim; sowie Kap. IV, 2.3.2.1, 2.3.2.3.

137 Zu den Aufständen von 1813: SpertKamp, Protest, S. $720 f$.; Wolfram Bell, Der Aufstand der »Knüppelrussen« im Bergischen Land, in: DreHER, ENGELBRECHT, Das Herzogtum Berg, S.85-92; Kandil, Sozialer Protest, S.52f., 72f., 101f.; Sergueï N. IskJul', Der Aufstand im Großherzogtum Berg gegen Napoleon im Jahre 1813, in: Zeitschrift des Bergischen Geschichtsvereins 92 (1986) S.57-68; Heinz RosentHal, Der bergische Aufstand 1813, in: Hildener Jahrbuch (1965-1960) S.197-206; Karl ScHRöDER, Der Aufstand gegen die französische Herrschaft in den Kantonen Waldbröl, Homburg und Eitorf zu Beginn des Jahres 1813, in: Romerike Berge 27 (1977) S. 119-132; DeRs., Der Aufstand gegen die französische Herrschaft in den Kantonen Waldbröl, Homburg und Eitorf zu Beginn des Jahres 1813, in: Romerike Berge 29 (1979) S.1-7; SchmidT, Grand-Duché, S. 459f. Siehe außerdem folgende Werke aus der Protestforschung zur Frühen Neuzeit: Peter BLICKLE, Deutsche Untertanen. Ein Widerspruch, München 1981; Winfried ScHulzE, Bäuerlicher Widerstand und feudale Herrschaft in der frühen Neuzeit, Stuttgart, Bad Cannstatt 1980; Andreas Würgler, Unruhe und Öffentlichkeit, Tübingen 1995; DerS., Modernisierungspotential; SUTER, Kulturen; DERS., Histoire sociale et événements historiques - Pour une nouvelle approche, in: Annales. Histoire, Sciences Sociales 52 (1997) 3, S.543-567; Mark HÄberlein (Hg.), Devianz, Widerstand und Herrschaftspraxis in der Vormoderne. Studien zu Konflikten im südwestdeutschen Raum (15.-18. Jahrhundert), Konstanz 1999; Trossвасн, Bauernprotest; Mooser, Rebellion; kritische Würdigung zu Blickle: Press, Herrschaft.

138 Bericht Beugnots an Roederer für die Monate Oktober, November und Dezember 1812, AN, 29 AP 38. 
Losziehung zunächst ruhig an ${ }^{139}$. Als der Elberfelder Unterpräfekt Schleicher jedoch am 22.Januar 1813 in der lutherischen Kirche in Ronsdorf mit der Ziehung der Konskribierten für die im Kanton Solingen gelegenen Munizipalitäten Remscheid und Cronenberg beginnen wollte, kam es zu tumultartigen Auseinandersetzungen. Konskribierte und betrunkene Arbeiter versperrten mit Eisenstöcken bewaffnet und unter »Hurra «-Rufen den Eingang der Kirche, zerschlugen Scheiben und Bänke des Gotteshauses und beschädigten anschließend das Haus des Maires. Schleicher ließ sich von den Ausschreitungen der Einwohner nicht an der Durchführung des Konskriptionsgeschäftes hindern. Trotz der Gewalttätigkeiten brachte er die Losziehung zum Abschluß ${ }^{140}$. Das Szenario wiederholte sich, als der Unterpräfekt am nächsten Tag die Auslosung der Wehrfähigen in Solingen fortzusetzen versuchte. Kassen, Ume und Möbel wurden zerstört. Der Unterpräfekt war gezwungen, den Saal zu verlassen und den Präfekten Spee zur Hilfe zu rufen. Die Anwesenheit des Departementleiters brachte die Aufständischen jedoch ebensowenig zur Ruhe. Sie ließen sich selbst dadurch nicht einschüchtern, daß Spee in militärischer Begleitung erschien. Da die Auslosung weiterhin boykottiert wurde, gaben Spee und Schleicher die Duchführung des Konskriptionsgeschäfts auf ${ }^{141}$.

In den Folgetagen weiteten sich die Konflikte wie ein Lauffeuer auf andere Gegenden des Großherzogtums aus. Hauptschauplatz der Auseinandersetzungen war hierbei das Rheindepartement, d.h. das Gebiet des ehemaligen Herzogtums Berg. Bis auf wenige Ausnahmen wurden alle Kantone des Departements von Insurrektionen erschüttert ${ }^{142}$. Selbst in Bezirken, in denen die Truppenaushebung bis zu den Ereignissen im Kanton Solingen ruhig abgewikkelt worden war, kam es im nachhinein zum Aufruhr ${ }^{143}$. Darüber hinaus entfachten auch im Siegdepartement Proteste. Sie waren aber im Gegensatz zum Rheindepartement weniger flächendeckend. Von den Unruhen wurde in erster Linie die ehemalige Herrschaft Gimborn-Neustadt im Norden des Departements mit ihren Kantonen Gummersbach, Waldbröl und Eitorf erfaßt ${ }^{144}$. Der südliche Landesteil blieb von den Ereignissen bis auf den Kanton Herborn verschont. Demgegenüber legte das Ruhrdepartement, wie Nesselrode am 6. März 1813 mit Nachdruck betonte, Zeugnis der größten Ordnung ab ${ }^{145}$.

139 Bericht des Generalprokurators Sethe an Roederer vom 14. April 1813, AN, AF IV 1840.

140 Bericht des Elberfelder Unterpräfekten an den Innenminister vom 22. Januar 1813, AN, AF IV 1226.

141 Bericht des Generalprokurators Sethe an Roederer vom 14. April 1813, AN, AF IV 1840.

142 Ibid.

143 Schreiben Nesselrodes an Roederer vom 24. Februar 1813, AN, 29 AP 39.

144 Bericht des Generalprokurators Sethe an Roederer vom 14. April 1813, AN, AF IV 1840; Bericht des Domänenempfängers Brewer an den Düsseldorfer Generaldirektor vom 29. Januar 1813, HStAD, GB, Nr.9553; Bericht des Eitorfer Rentmeisters an den Dillenburger Domänendirektor vom 19. Februar 1813, ibid.

145 Bericht Nesselrodes an Roederer vom 6. März 1813, AN, 29 AP 39. 
Anzeichen von Unruhe waren lediglich in den drei an das Rheindepartement angrenzenden Kantonen der ehemaligen Grafschaft Mark Schwelm, Hagen und Lüdenscheid erkennbar. Die übrigen Teile des Departements blieben von der Insurrektion unberührt ${ }^{146}$.

Die Aufständischen gingen meist nach dem gleichen Schema vor. Sie entluden ihre Aggressionen in erster Linie an Herrschaftssymbolen. Unter der Losung Vivent les russes, vive Alexandre ${ }^{147}$ wurden Gemeindediener bedroht, Konskriptionslisten eingezogen, Verwaltungsakten wie die Gesetz-Bulletins und Zivilstandsregister der Mairien, die Tabellen der Tabak- und Salzdebitanten und das Stempelpapier der Domänenverwaltung zerstört, Kassen und Tabak- bzw. Salzdepots geplündert, Amtsgebäude und Häuser von Staatsdienern, allen voran die der Munizipalbeamten, Zolleinnehmer und Salz- und Tabakdebitanten, verwüstet oder in Brand gesetzt ${ }^{148}$ sowie Gefangene befreit ${ }^{149}$. Der Sachschaden an öffentlichem und privatem Eigentum war beträchtlich. Einzelne Banden ließen sich bei Gemeindebewohnern einquartieren oder erpreßten von diesen Geld und Waffen ${ }^{150}$. Im Siegdepartement besaßen die Unruhen darüber hinaus starke restaurative Züge. Hier tauchte nicht nur das Herrschaftszeichen des ehemaligen Landesherrn, wie zum Beispiel die orangefarbene Kokarde, wieder auf. In Herborn etwa wurde eine Krone aus organgefarbenenen Blättern auf den Kirchturm gesteckt ${ }^{151}$. Hier unterzeichnete der Gummersbacher Friedensrichter Pollmann ebenfalls ein von den Aufständischen verfaßtes und am 30.Januar 1813 veröffentlichtes Papier, mit dem er in seine alte Funktion als Vogt gesetzt, die neue Ordnung aufgehoben und alte Rechte, insbesondere die Freiheit der Jagd und des Fischens, wiederhergestellt wurden. Pollmann versprach außerdem, die ehemaligen Schöffen und Vorsteher wieder einzusetzen. Im Gegenzug dafür sollten die Einwohner Ruhe und Ordnung wiederherstellen und den Besitz unberührt lassen ${ }^{152}$.

146 Bericht des Generalprokurators Sethe an Roederer vom 14. April 1813; Bericht des Präfekten Romberg an Roederer vom 15. Februar 1813, AN, AF IV 1840.

$147 \mathrm{Vgl}$. den Bericht des Generalprokurators Sethe an Roederer vom 14. April 1813, ibid.

148 Zum Beispiel zündeten Aufständische in Lindlar das Haus des Polizeisergeanten an. Siehe den Bericht des Lindlarer Beigeordneten vom 13. März 1813, HStAD, GG, Nr.76.

149 In Elberfeld etwa befreiten die Aufständischen einen Vagabunden und eine Tabakdebitantin. Siehe das Schreiben des Elberfelder Friedensrichters vom 31.Januar 1813, HStAD, GG, Nr.1222. Zu den Angriffen der Aufständischen vgl. Beugnots Brief an Roederer vom 31. Januar 1813, AN, 29 AP 38, den Bericht des Domänenempfängers Brewer an den Düsseldorfer Generaldirektor vom 29. Januar 1813, HStAD, GB, Nr.9553, sowie das Schreiben des Lindlarer Beigeordneten Court vom 24. Mai 1813, HStAD, GG, Nr.76.

150 So etwa in Elberfeld. Bericht Nesselrodes an Roederer vom 29. Januar 1813, AN, 29 AP 39; Schreiben des Elberfelder Friedensrichters vom 31.Januar 1813, HStAD, GG, Nr. 1222.

151 Vgl. den Bericht des Generalprokurators Sethe an Roederer vom 14. April 1813, AN, AF IV 1840, ferner das Schreiben des Innenministers an Roederer vom 29.Januar 1813, AN, 29 AP 39.

152 Arrêt des Düsseldorfer Appellationshofs vom 1. April 1813, AN, AF IV 1840; Projet de rapport sur les operations des autorités judiciaires à l'occasion des troubles (Februar 1813), AN, 29 AP 37. 
Über die Zahl der Aufständischen lassen sich keine sicheren Angaben machen. Die von den örtlichen Amtsträgern gemachten Schätzungen stellen keine zuverlässige Quelle dar. Es ist anzunehmen, daß sie unter dem Eindruck der Vorgänge häufig überzogen waren. So soll sich die Zahl der Aufständischen bei der Konskriptionsziehung in Siegen den Angaben des Präfekten Spee zufolge auf 3000 bis 4000 Mann belaufen haben ${ }^{153}$. Fest steht nur, daß die Insurrektionen hauptsächlich von Konskribierten, Deserteuren, erwerbslosen Arbeitern, Handwerkern und Gastwirten getragen wurden - von jenen Bevölkerungsgruppen also, die am meisten unter den Folgen der napoleonischen Herrschafts- und Reformpolitik litten, aber ebenfalls die geringsten Mitsprachemöglichkeiten besaßen ${ }^{154}$. Im Siegdepartement beteiligten sich auch Bauern an den Aufständen ${ }^{155}$. Nur gelegentlich traten Mitglieder der Honoratiorenschaft als Akteure in Erscheinung. Der als Kandidat für die Notabelnliste vorgeschlagene ehemalige Maire Lindlars, Klug, etwa beherbergte die Aufständischen in seinem Haus und schrieb zudem Vivat Russen auf Türen und Fenster ${ }^{156}$. Andere wiederum förderten das Vorgehen der Aufständischen mittelbar, indem sie sich, wie etwa der Lindlarer Pastor, neutral verhielten oder den Kommunalbediensteten keinen bewaffneten Beistand leisteten ${ }^{157}$. Ansonsten ist nichts darüber bekannt, daß sich Mitglieder der lokalen Eliten an den Gewalttätigkeiten beteiligten. Auch die Mehrzahl der Staatsdiener verhielt sich der französischen Regierung gegenüber loyal. Selbst jene Bediensteten, die sich vor den Insurgenten versteckten, wie die Leiter der Mairien Duisburgs und Mülheims am Rhein ${ }^{158}$, andere Beamte als Stellvertreter einsetzten ${ }^{159}$, ihre Dienstposten verließen, so etwa der Unterpräfekt des Siegener Arrondissements, Herrmann ${ }^{160}$, oder gar den Forderun-

153 Bericht des Präfekten Spee an den Innenminister vom 23.Januar 1813, AN, AF IV 1226.

154 Vgl. das Schreiben des Innenministers an Roederer vom 29. Januar 1813, AN, 29 AP 39, ferner die Wöchentliche Tabelle derjenigen Vergehen und Verbrechen, welche [...] bei dem Tribunal zu Hagen zur Anzeige und Untersuchung gekommen sind (1813), StAM, GB, A2, Nr. 3 .

155 Bericht des französischen Geheimagenten vom 23. Februar 1813 an den französischen Polizeiminister Savary, AN, 29 AP 38; vgl. auch ScHMIDT, Grand-Duché, S. 463.

156 Siehe den Bericht des Lindlarer Beigeordneten vom 13. März 1813, HStAD, GG, Nr.76. Der Mülheimer Unterpräfekt Spiess empfahl dem Präfekten Spee aus diesem Grund, Klug aus der Notabelnliste zu streichen. Vgl. das Schreiben des Unterpräfekten Spiess an Spee vom 9. Juni 1813, HStAD, GB, Nr. 9756.

157 Bericht des Lindlarer Beigeordneten vom 13. März 1813, HStAD, GG, Nr. 76.

158 Bericht Nesselrodes an Roederer vom 6. Februar 1813, AN, 29 AP 39.

159 Der Maire von Mülheim an der Ruhr ließ sich von seinem ersten Beigeordneten vertreten. Der Gummersbacher Maire Heuser überließ Friedensrichter Pollmann die Leitung seiner Kommune. Vgl. den Bericht Nesselrodes an Roederer vom 6.Februar 1813, ibid., sowie den Arrêt des Düsseldorfer Appellationshofs vom 1. April 1813, AN, AF IV 1840.

160 Bericht Nesselrodes an Roederer vom 6. Februar 1813, AN, 29 AP 39. Auch der Wermelskirchener Maire Arnold Frowein gab seine Stellung auf. Siehe das Schreiben des Prä- 
gen der Aufständischen nachgaben - das exponierteste Beispiel hierfür bietet der Fall Pollmann -, taten dies wohl nicht aus Illoyalität oder Sympathie für die Aufständischen, sondern in erster Linie unter dem Eindruck bzw. dem Druck der Ereignisse. Daß die Verwaltungsdiener hierbei die tatsächliche Gefahr überschätzten, ist wahrscheinlich, zumal sie erstmals mit einem solchen Problem konfrontiert wurden. Das meinten im nachhinein auch Mitglieder der Regierung. La frayeur et la faiblesse de la plupart des administrateurs, verurteilte General Damas das Verhalten der subalternen Beamten am 21. März 1813, ont presque partout fait la force des revoltés. [...] On a [...] trop exageré le danger ${ }^{161}$.

Der Ausbruch von Protesten traf die Regierung nicht ganz unvorbereitet. Die Siege der russischen Truppen wie auch die durchzuführende Konskriptionsziehung hatten die Düsseldorfer Minister seit Beginn des Jahres zur Wachsamkeit veranlaßt und zum vorsorglichen Erlaß einzelner Präventivmaßnahmen geführt. Mitte Januar 1813 hatten die Ressortleiter Säuberungsaktionen unter den ehemaligen Mitgliedern des preußischen Militärs eingeleitet und die verschärfte Überwachung der Bevölkerung durch die Unterbehörden angeordnet. Darüber hinaus hatten sie in Paris um militärische Verstärkung gebeten ${ }^{162}$. Les mesures sont prises, beruhigte Beugnot Roederer in der zweiten Januarhälfte 1813, pour qu'on sache sous quelques jours s'il coure du feu sous la cendre ${ }^{163}$.

Dennoch hatten die Minister nicht wirklich mit dem Ausbruch von Unruhen gerechnet. Le Grand Duché parait en général tranquille, berichtete Beugnot noch unmittelbar vor Ausbruch der Unruhen seinem Vorgesetzten nach Paris $^{164}$. Noch weniger hatten sie erwartet, daß das Rheindepartement Ausgangspunkt von Protesten werden würde. Die Möglichkeit einer Revolte bestand für sie allenfalls in der ehemaligen Grafschaft Mark, weshalb sie dort über die landesweit verschärften Kontrollmaßnahmen hinaus zusätzlich einen Geheimagenten einsetzten ${ }^{165}$. Sobald die geringste Möglichkeit zum Aufruhr bestehe, prophezeite Beugnot am 15. Januar 1813, elle commencera dans le Comté de la Mark ${ }^{166}$. Die ehemalige preußische Provinz galt als die politisch unzuverlässigste. Ihre Bevölkerung stand in dem Ruf, sich des Reichsfreiherrn vom und zum Stein, der dort mehrere Jahre gewirkt hatte, noch immer eng verbunden zu fühlen. Aufgrund ihrer langjährigen Zugehörigkeit zu Preußen

fekten Spee an Beugnot vom 15.Juni 1813, HStAD, GB, Nr.144; vgl. ferner SchröDER, Aufstand, S. 122.

161 Schreiben des Generals Damas vom 21. März 1813, AN, 29 AP 40.

162 Beugnot in seinem Bericht an Roederer vom 15.Januar 1813, AN, 29 AP 38.

163 Beugnot in seinem Bulletin an Roederer für die dritte Januarwoche 1813, ibid.

164 Ibid.

165 Darauf weist Beugnot in seinem Bericht an Roederer vom 15. Januar 1813 hin, ibid.; vgl. ebenfalls den Bericht eines Agenten Beugnots im Großherzogtum Berg über die Situation im Ruhrdepartement vom 26. November 1812, ibid.

166 Beugnot in seinem Bericht an Roederer vom 15.Januar 1813, ibid. 
galten die märkischen Einwohner zudem nach wie vor als preußenanhängig ${ }^{167}$. Allerdings hatten die Behörden selbst in der Mark das Unruhepotential in der Einwohnerschaft für zu gering gehalten, um Proteste auslösen zu können. Aufstände seien nicht zu befürchten, meinte Romberg Mitte Januar 1813, pourvu qu'il ne survienne d'impulsions de vive force de l'exterieur [sic] ${ }^{168}$.

Nesselrode und Beugnot, die aufgrund ihrer Fehleinschätzung der Stimmungslage in der Bevölkerung sichtlich erschüttert waren, als sie von den Gewalttätigkeiten im Kanton Solingen erfuhren, ${ }^{169}$ forderten noch am 24. Januar 1813 militärische Unterstützung aus Frankreich zur Eindämmung der Unruhen an ${ }^{170}$. Mit Hilfe des französischen Adjutanten Jean Léonor François Lemarois (1776-1836) ${ }^{171}$, den die Pariser Regierung wohl in Reaktion auf den Bericht eines Spions mit den Aufgaben der Hohen Polizei in Düsseldorf betraute ${ }^{172}$, wurde der Aufstand schließlich niedergeschlagen. Am 6. Februar 1813, also immerhin mehr als zwei Wochen später, setzte Beugnot Roederer davon in Kenntnis, daß die Revolte beendet worden sei ${ }^{173}$.

In einigen Kommunen hatte es die Regierung nicht dem Militär, sondern der Initiative und dem gemeinschaftlichen Engagement von Gemeindebewohnern und subalternen Beamten zu verdanken, daß die Aufstände so schnell eingedämmt werden konnten. In Wipperfürth, Eitorf und Schwelm beispielsweise gelang es den Kommunalvorstehern, Einwohner ihrer Verwaltungsbezirke für den bewaffneten Kampf gegen die Revoltierenden zu gewinnen und diese ohne fremde Hilfe in die Flucht zu schlagen. In Eitorf wurden dabei mehrere Aufständische verhaftet, zwei kamen zu Tode ${ }^{174}$. In Hagen und Lünen solidarisierten sich Munizipalbeamte und Einwohner mit der Gendarmerie und dem Unterpräfekten und traten gemeinsam mit diesen den Aufständischen entgegen. Fünfzehn Personen wurden dabei festgenommen und in die Dortmunder Ge-

167 Ibid.

168 Romberg in seinem Bericht über den Esprit public im Ruhrdepartement an Roederer vom 16. Januar 1813, AN, AF IV 1840.

169 Er komme nicht über die Unruhen im Kanton Solingen hinweg, gestand Beugnot Roederer in seinem Brief vom 24. Januar 1813, AN, 29 AP 38.

170 Schreiben Nesselrodes an Roederer vom 24. Januar 1813, AN, 29 AP 39.

171 Zur Person Lemarois' siehe Vincent Rolin, Les aides de camp de Napoléon et des maréchaux sous le Premier Empire 1804-1815, Cahors 2004, S. 184.

172 Welche Aufgaben Lemarois genau zu erfüllen hatte, war nicht ganz klar. Vgl. die Briefe Roederers an Beugnot vom 30. Januar bzw. 27. März 1813, AN, 29 AP 38. Beugnot hatte die Übertragung der Hohen Polizei auf einen französischen Staatsdiener seit langem befürchtet und abzuwenden versucht, nicht zuletzt, um nicht in seinem eigenen Wirkungskreis eingeschränkt zu werden. Vgl. dazu etwa das Schreiben des kaiserlichen Kommissars an Roederer vom 10. Dezember 1811, AN, AF IV 1840. Neben der Entsendung Lemarois' schickte Napoleon einen französischen Kriegskommissar nach Düsseldorf, um Nesselrode bei der Erledigung der militärischen Aufgaben zu unterstützen (Gesetz-Bulletin, 2. Abtheilung, Nr. 123).

173 Brief Beugnots an Roederer vom 6. Februar 1813, AN, 29 AP 38.

174 Schreiben des Innenministers an Roederer vom 29. Januar 1813 AN, 29 AP 39; Schreiben des Innenministers an Roederer vom 5. Februar 1813, AN, AF IV 1840. 
fängnisse gebracht. Die übrigen ergriffen die Flucht ${ }^{175}$. Daß sich Einwohner und Verwaltungsdiener zusammenschlossen, um die Aufstände zu bekämpfen, geschah dabei wohl weniger aus Loyalität gegenüber der französischen Herrschaft als vielmehr aus dem gemeinsamen Interesse an der Wiederherstellung von Ruhe und Ordnung bzw. an der Sicherung ihres Eigentums.

Mit dem Ausbruch von Unruhen begann in den Behörden die hektische Suche nach den Gründen und den Verantwortlichen für diese Vorgänge. Die einheimischen Beamten neigten dazu, die französische Herrschaftspolitik für die Proteste haftbar zu machen. Während Zollverwalter David und Generalprokurator Sethe die Insurrektion in erster Linie auf die Salz- und Tabakregie zurückführten ${ }^{176}$, gab Innenminister Nesselrode der napoleonischen Wirtschafts- und Zollpolitik insgesamt Schuld an dem Aufstand. Er war der Meinung, die zunehmende Erwerbslosigkeit der Arbeiter habe vor allem in den bergischen Gewerbegebieten den Nährboden für die Unruhen geschaffen und die Protestbereitschaft der Gemeindebewohner gefördert ${ }^{177}$. Dagegen sah der Elberfelder Friedensrichter in den Insurgenten nichts als eine zusammen gelaufene Bande von wahrem Lumpengesindel [...], welche ohne Zweck und Plan, ohne eigentlichen Anführer durch die Orthschaften zog, und mehr eine Rauberei als politische Umwälzung zu intendiren schien ${ }^{178 .}$

Auch in Paris brachte man die Proteste mit der Tabak- und Salzregie in Verbindung, machte aber nicht das Monopol an sich für die Aufstände verantwortlich, sondern die Härte der Düsseldorfer Ministerialbürokratie bei der Abgabenerhebung ${ }^{179}$ - ein Vorwurf, den Roederer und Beugnot später dementierten ${ }^{180}$. Die im Großherzogtum tätigen französischen Staatsdiener Beugnot und Lemarois ließen sich demgegenüber von Vorurteilen leiten und favorisierten die Verschwörungstheorie ${ }^{181}$. Sie sahen in den Aufständen ein von Preußen geschmiedetes Komplott und in der Person des Freiherrn vom Stein den Drahtzieher der Aufstandes. Die Verschwörung sollte ihrer Meinung nach dazu dienen, auf deutschem Boden eine allgemeine Volkserhebung

175 Vgl. das Schreiben des Präfekten des Lippedepartements an den französischen Innenminister vom 2. Februar 1813, AN, F/7/3054, ferner das undatierte Schreiben Roederers an Napoleon (Februar 1813), AN, AF IV 1840.

176 Bericht des Zollverwalters David an Beugnot vom 12. Februar 1813, AN, AF IV 1840.

177 Monatsbericht Nesselrodes an Roederer für Januar 1813; Schreiben Nesselrodes an Roederer vom 16. Februar 1813, AN, 29 AP 37.

178 Schreiben des Elberfelder Friedensrichters vom 31.Januar 1813, HStAD, GG, Nr. 1222.

179 Bericht des Geheimagenten an den französischen Polizeiminister Savary vom 22. Februar $1813, \mathrm{AN}, 29 \mathrm{AP} 40$.

$180 \mathrm{Vgl}$. den Schriftwechsel zwischen Roederer und Beugnot in dieser Sache: Brief Roederers an Beugnot vom 9.Februar 1813; Antwortschreiben Beugnots an Roederer vom 16. Februar 1813, AN, 29 AP 37. Vgl. ebenfalls Roederers Bericht an Napoleon (April 1813), AN, 29 AP 40.

181 Beugnot in seinem Schreiben an Roederer vom 5. Februar 1813; vgl. ferner den Bericht des Generals Lemarois an Napoleon vom 22. Januar 1813, AN 29 AP 37. 
einzuleiten. Je vous ai dit, schrieb Beugnot Roederer am 5. Februar 1813, que ce mouvement partait de haut, et que c'etait le Baron de Stein qui le dirigeait; et qu'il ne s'agissait pas ici des émeutes qui s'excitent et s'appaisent au hazard, mais d'un plan d'insurrection bien combiné $e^{182}$.

Beugnot und Lemarois schätzten aber den Charakter der Revolte falsch ein. Es handelte sich ebensowenig um eine preußische Intrige wie um das Fanal einer allgemeinen Erhebung der Volksmassen. Drei Gründe sprechen dagegen. Erstens besaßen die Proteste nur einen geringen Organisationsgrad. Die Insurgenten schlossen sich spontan und willkürlich zu Banden zusammen. Klare hierarchische Strukturen besaßen ihre kurzfristig eingegangenen Bündnisse nicht und auch keinen festen inneren Zusammenhalt. Zweitens bestanden zwischen den einzelnen Konfliktherden nur lose Verbindungen. Zwar waren die einzelnen Gruppen über die Vorgänge in anderen Munizipalitäten informiert und wurden dadurch sicherlich zum Handeln angeregt. Kohärenz gab es zwischen ihnen freilich nicht. Jede Gruppe ging in der Regel eigenständig vor. Die Konfliktherde blieben örtlich begrenzt. Drittens machten die Aufständischen mit ihren Angriffen auf Repräsentanten, Institutionen und Symbole der französischen Herrschaft in erster Linie ihrem Unmut und ihrer Empörung über konkrete Aspekte der französischen Herrschaft, wie etwa der Konskription und der ungeliebten Steuer- und Wirtschaftspolitik, Luft. Aus diesem Grund beteiligten sich vor allem solche Personen an den Protesten, die von den Folgen dieser Politik aus der Bahn geworfen worden waren. Deshalb brachen die Unruhen auch in den Territorien des Großherzogtums aus, die unter den Folgen der französischen Hegemonialpolitik besonders litten: den Gewerbegebieten.

Politische Überzeugungen oder Motive lagen dem Aufruhr hingegen meist nicht zugrunde. Vielmehr handelte es sich bei den Eruptionen um spontane Reaktionen auf die strukturellen Wandlungsprozesse von Staat und Gesellschaft sowie deren soziale Verwerfungen. In erster Linie wurde der sich ausdehnende Staat attackiert und nicht die französische Herrschaft ${ }^{183}$. Insofern bestand eine längerfristige Kontinuitätslinie zu den Widerstandsformen und Protestaktionen, die das Werden des modernen Staates in der Frühen Neuzeit begleitet hatten. Um die Abschüttelung der französischen Herrschaft ging es daher auch nicht, wenngleich vereinzelt Pamphlete zu diesem Zweck zirkulierten ${ }^{184}$. Die Idee der Nation tauchte ohnehin nirgendwo auf. Losungen wie "Vive Alexandre« dürfen in diesem Rahmen nicht überbewertet bzw. mißverstanden werden. Sie dienten der Integration nach innen und Legitimation bzw. Provokation nach außen, besaßen aber keine politische Stoßrichtung. Glei-

182 Beugnot in seinem Schreiben an Roederer vom 5. Februar 1813, ibid.

183 Eine ausgezeichnete Analyse der Protestursachen in napoleonischer Zeit bietet SPErTKamp, Protest, S.722f.

184 Vgl. die beiden Proklamationen an die Bevölkerung, die dem Schreiben Beugnots an Roederer vom 31. Januar 1813 beilagen, AN, 29 AP 38. 
ches galt für die restaurativen Tendenzen der Aufstände im Siegdepartement. Dem Rückgriff auf die Herrschaftszeichen der vormaligen Landesherren lagen wohl weniger konkrete Absichten zur Restauration der Herrschaft Nassau-Oraniens als eher der »Mythos einer guten, stabilen und vor allem friedlichen Ordnung « ${ }^{185}$ zugrunde. Das war selbst in Gummersbach der Fall, wo die alte Ordnung tatsächlich wiederhergestellt wurde.

Politisch wirkungslos blieben die Aufstände dennoch nicht. Zum ersten hob die Regierung nach Beendigung der Unruhen das Tabakmonopol auf, das eine Zielscheibe der Angriffe während der Proteste gewesen war ${ }^{186}$. Allerdings blieben Anbau, Herstellung und Verkauf von Tabak verboten ${ }^{187}$. Zum zweiten führten die Aufstände den Behörden vor Augen, wie sehr die soziale Ordnung durch Arbeiter gefährdet war, denen es an Brot und Arbeit fehlte. Zum dritten - und darin lag wohl die wichtigste Bedeutung des Aufruhrs - legten die Protestteilnehmer, wie Beugnot schon im Frühjahr befürchtet hatte ${ }^{188}$, langfristig den Keim für die Destabilisierung und Desintegration des Großherzogtums. Wirklich zur Ruhe kam der Staat nach den Unruhen nicht mehr. Deserteure und Refraktäre zogen in Banden durchs Land, griffen Gendarmen an und diskreditierten die Staatsgewalt ${ }^{189}$, ohne daß die Regierung diesen Entwicklungen Einhalt zu bieten wußte. Obwohl sie zunehmend repressiver werdende Maßnahmen ergriff, glitten ihr die Verhältnisse mehr und mehr aus der Hand ${ }^{190}$. Mit dem schwindenden Vertrauen der Bevölkerung in den Staat ging ein Autoritätsverlust der staatlichen Institutionen einher.

Il n'est pas permis, stellte Beugnot gegenüber Roederer Mitte Mai 1813 resigniert fest, de dire que l'Etat du Grand-Duché soit satisfaisant. Il le sera quand les déserteurs seront repris et le contingent de 1813 remplis; que les contributions s'y leveront sans plus de frais que l'an passé; que toute route sera sure, toute propriété respectée; tout individu occupé et tranquille ${ }^{191}$.

185 SpeitKamp, Protest, S. 725.

186 Das Monopol wurde am 21. Februar 1813 aufgehoben. Das diesbezügliche Dekret befindet sich in der Akte AN, AF 1853B. Nesselrode hatte sich anfangs gegen die Aufhebung der Tabakregie ausgesprochen. Vgl. dazu das Schreiben Nesselrodes an Roederer vom 17. Februar 1813, AN, 29 AP 39.

187 Gesetz-Bulletin, 2. Abtheilung, Nr.126.

188 Solange sich diese Arbeiter zwischen Aufruhr und Hunger befänden, hatte er gegenuber Roederer in seinem Schreiben vom 18. Februar 1813 prognostiziert, je dis que l'ordre pourra bien être rétabli mais ne sera point garanti; je dis qu'il ne sera pas inébranlable (AN, 29 AP 38).

189 Vgl. etwa den Geheimen Bericht Beugnots an Roederer für März 1813, ibid., ferner das Schreiben Beugnots an Roederer vom 29. März 1813, AN, 29 AP 39, das Schreiben des Driedorfer Maires an den Präfekten Schmitz vom 17.Juni 1813, HStAW, Abt.370 Großherzogtum Berg, Nr.1538, sowie den Aufruf zum Aufstand vom 30. März 1813, den der Gastwirt Karl Schade in Iserlohn heimlich veröffentlicht haben soll, AN, AF IV 1840.

190 Um die drastisch zunehmende Desertion zu bekämpfen, verfügte Nesselrode in einem undatierten Arrêt (März 1813), sämtliche Militärpersonen, die sich außerhalb ihrer Mairien aufhielten, zu verhaften und mit dem Tod zu bestrafen, AN, AF IV 1226.

191 So Beugnot in seinem Bulletin an Roederer vom 15. Mai 1813, AN, 40 AP 17. 


\subsection{Justizwesen}

\subsubsection{Neuordnung der Justizverfassung}

Neben der Ausgestaltung der Exekutive war die Reorganisation der Justiz eines der Hauptanliegen der französischen Verfassungspolitk in Berg. Hierbei ging es zunächst nur um die konstitutionelle Verankerung der Justiz, obwohl Agar bereits im März 1806 die Verquickung von Verwaltung und Rechtsprechung wie auch die Kostspieligkeit der Gerichtsverfahren anprangerte und Reformen in diesen Punkten nahelegte ${ }^{192}$. Der Verfassungsentwurf, den der neue Landesherr Ende März 1806 nach Paris sandte, enthielt keine grundsätzlichen Neuerungen für die Justizorganisation.

Es ist bereits darauf hingewiesen worden, daß der französische Kaiser die Hast verurteilte, mit welcher Joachim Murat seine konstitutionellen Pläne durchzusetzen suchte. Um Aussagen über die Justiz machen zu können, hielt er es für erforderlich, zunächst eine Bestandsaufnahme der tradierten Verhältnisse zu machen. Vor allem mußte geprüft werden, ob ausländische Gerichte für das Großherzogtum zuständig waren. Il est probable, teilte Napoleon Murat am 4. April 1806 mit, qu'on allait en dernier appel à Munich, peut être même aux tribunaux de l'Empire; il faut abolir tout cela, et que tout se termine en dernier appel à Dusseldorf ${ }^{193}$.

Während Napoleon nichts überstürzen wollte, nutzte Murat die sich ihm bietende Chance zur Reform, ohne Zeit zu verlieren. Zusammen mit der Neuordnung der Oberbehörden entzog er den preußischen Regierungen von Münster und Berlin am 24. April 1806 die Zuständigkeit für die klevischen Einwohner und übertrug diese den beiden Obergerichten des Herzogtums Berg - einer der wenigen Fälle, in dem man auf französischer Seite bergische Strukturen als Vorlage für die Organisation des gesamten Landes benutzte ${ }^{194}$. Die bergischen Obergerichte übernahmen zugleich die Prozesse, die bei den Gerichtshöfen von Münster und Berlin anhängig waren. Auch die von den Reichsgerichten in dritter Instanz ausgeübte Gerichtsbarkeit für die Tribunale in Limburg, Rheda und Dortmund ging auf den Düsseldorfer Oberappellationshof über ${ }^{195}$. Die in intermediärer Hand liegenden Gerichtsbarkeiten tastete Murat indes nicht an, zumal die jurisdiktionellen Befugnisse den mediatisierten Fürsten in der Rheinbundakte als Standesrechte zugesichert worden waren ${ }^{196}$.

Als der bergische Staatsrat im Frühjahr 1807 entschied, die heterogene Rechtslage in den verschiedenen Teilen des Landes durch den Transfer der französischen Zivilgesetzgebung zu beseitigen, setzten in Düsseldorf erstmals

192 Agar in seinem undatierten Tableau de l'administration civile et judiciaire des duchés de Clèves et de Berg sous le régime prussien (März 1806), AN, AF IV 1225.

193 Napoleon in seinem Schreiben an Joachim Murat vom 4. April 1806, AN, 31 AP 24.

194 Verfügung Murats vom 24. April 1806, ADD, Fonds Mosbourg, 100 MI 115 R2.

195 Beylage zu Nr.19 der Großherzoglich-Bergischen Wöchentlichen Nachrichten, 13.Mai 1806.

196 Art. 27 der Rheinbundakte; ClERCQ, Recueil, S. 177. 
Diskussionen über die grundlegende Reorganisation der Justiz ein ${ }^{197}$. Der Code Napoléon beruhte nämlich auf einer Gerichtsverfassung, die es im Großherzogtum nicht gab. Obgleich die Einführung des französischen Zivilrechts die Assimilierung der Rechtsstrukturen an französische Verhältnisse nahelegte, scheint zunächst offen gewesen zu sein, wie die künftige Justizverfassung konkret aussehen sollte. Daß das bergische Gerichtswesen tatsächlich nach französischem Beispiel organisiert werden sollte, ergab sich erst, als Murat zu Beginn des Jahres 1808 beschloß, dem Großherzogtum Berg eine der westfälischen Konstitutionsakte nachgebildete Verfassung zu oktroyieren. Die erste Kommission des bergischen Staatsrats wurde daraufhin damit beauftragt, die Einführung der französischen Justizverfassung im Großherzogtum vorzubereiten.

Die Kommissionsmitglieder machten sich umgehend an die Arbeit und reichten schon in den Folgemonaten erste Vorschläge ein. Dennoch kam es während der Herrschaft Murats nicht mehr zur geplanten Erneuerung der Justizorganisation, weil Finanzminister Agar grundsätzliche Vorbehalte gegen das Projekt hatte und dieses deshalb nicht entsprechend vorantrieb. Er befürchtete, daß die ehemaligen Justizbeamten in der Zeit, die zwischen gesetzlichem Erla $\beta$ und tatsächlichem Inkrafttreten der neuen Ordnung lag, ihre Aufgaben nicht gewissenhaft erfüllten und sich daraus Nachteile für die Rechtsprechung ergäben ${ }^{198}$. Hinzu kam, daß Murat auch die Justizreform nicht mehr mit dem nötigen Nachdruck verfolgte, nachdem er erfahren hatten, daß Napoleon den Thron des Großherzogtums neu zu besetzen plante.

Als der französische Kaiser im Sommer 1808 die Herrschaft im Großherzogtum Berg antrat, griff er das Reformprojekt seines Vorgängers schnell wieder auf. Schon am 11. September 1808 ließ er Beugnot über seine Absicht informieren, die französische Gerichtsverfassung auf Berg zu übertragen, und beauftragte diesen damit, einen entsprechenden Organisationsentwurf vorzubereiten ${ }^{199}$. Napoleons Reformbestrebungen verliefen jedoch zunächst im Sande, weil der kaiserliche Kommissar es für sinnvoller hielt, zuerst die Gesetzgebung und dann die Justizverfassung zu reformieren ${ }^{200}$. Er arbeitete daher keinen Entwurf zur Übernahme der französischen Gerichtsorganisation aus, sondern beschränkte sich darauf, dem Kaiser Vorschläge zur Einführung des französischen Zivilrechts zu präsentieren ${ }^{201}$. Anscheinend beließ man es in Paris zunächst hierbei, denn Napoleon unterzeichnete im Herbst 1809 das

197 Schreiben Agars an Joachim Murat vom 2. April 1807, ADD, Fonds Mosbourg, 100 MI 118; vgl. auch Francksen, Staatsrat, 47f.; ROB, Regierungsakten des Großherzogtums Berg, S. 167f.

198 Schreiben Agars an Joachim Murat vom 2. Februar 1808, ADD, Fonds Mosbourg, 100 MI 119.

199 Anweisung Napoleons an Gaudin vom 11. September 1808, AN, AF IV 1886A.

200 Antwortschreiben Beugnots an den Pariser Finanzminister Gaudin vom 7. September 1808, AN, AF IV 1842.

201 RoB, Regierungsakten des Großherzogtums Berg, S. 177 f. 
Dekret zur Einführung des Code Napoléon, ohne zugleich die notwendigen Voraussetzungen in der Gerichtsverfassung zu schaffen.

Durch den Beschluß, den Code Napoléon am 1. Januar 1810 in Kraft treten zu lassen, kamen die Planungen zum Transfer der französischen Justizverfassung allerdings wieder in Gang. Anfang November 1809 trat Minister-Staatssekretär Maret an Beugnot heran und forderte ihn nunmehr ultimativ dazu auf, endlich den seit langem in Aussicht gestellten Entwurf zur Reorganisation der Justizverfassung vorzulegen. Um die Neuordnung der Gerichte noch zusammen mit der Einführung des Code Napoléon vollziehen zu können, sollte die Arbeit noch im laufenden Monat in Paris eingehen ${ }^{202}$.

Im Unterschied zum Sommer 1808 reagierte Beugnot dieses Mal unverzüglich auf den Befehl aus Paris. Anfang Dezember 1809 ließ er Maret die gewünschten Unterlagen zukommen ${ }^{203}$. Diese enthielten neben einer von ihm und Nesselrode gemeinsam entworfenen Dekretvorlage zur Übernahme der französischen Gerichtsorganisation ${ }^{204}$ zugleich die zur Besetzung der neuen Richterstellen erforderlichen Personallisten ${ }^{205}$. Maret war mit den Vorschlägen der beiden Ressortchefs nicht zufrieden und sandte die Entwürfe zur Überarbeitung umgehend nach Düsseldorf zurück. Wiewohl Beugnot betont hatte, $\mathrm{da} B$ die von Nesselrode und ihm entworfene Justizorganisation vollständig dem französischen Beispiel nachgebildet war ${ }^{206}$, ging dem Minister-Staatssekretär die Analogie zwischen der Vorlage der beiden Ressortleiter und dem französischen Modell nicht weit genug. Seiner Meinung nach mußte die Gerichtsverfassung Bergs dem Vorbild des Empire getreu nachgebildet werden, denn für ihn war das Großherzogtum ein Land, qui ne doit être régi par une partie des loix de l'Empire, mais par toutes ${ }^{207}$. Abweichungen vom französischen Modell, wie sie die beiden Düsseldorfer Minister mit Blick auf die Andersartigkeit der lokalen Verhältnisse in Berg vorgenommen hatten, hielt er demnach nicht für statthaft. Dies galt einmal für die Preisgabe der flächendeckenden Einführung von Gerichten der ersten Instanz auf Arrondissementebene und die Vergrößerung des Gerichtspersonals, die die Düsseldorfer Minister beantragt hatten. Es galt gleichfalls für den Verzicht auf die Einreichung dreifacher Kandidatenlisten ${ }^{208}$.

Innenminister Nesselrode stimmte dem französischen Minister-Staatssekretär nicht zu. Er lehnte die vollständige Assimilierung der bergischen Gerichtsordnung an das französische Modell weiterhin ab. Nicht zuletzt aufgrund der

204 Der Staatsrat wurde nicht daran beteiligt.

205 Für die Arbeit zeichnete laut Beugnot in erster Linie der Innenminister verantwortlich. Dieser hatte sich offenbar schon seit längerem mit der Justizreform beschäftigt.

206 In seinem diesbezüglichen Anschreiben vom 1. Dezember 1809 versicherte der kaiserliche Kommissar dem Minister-Staatssekretär, darauf bestanden zu haben, qu'il fut entièrement calqué sur la loi française, ibid.

207 Schreiben Marets an Beugnot vom 7. Dezember 1809, ibid.

208 Ibid. 
geringen Größe des Großherzogtums hielt er es für unvermeidlich, örtliche Partikularitäten im Aufbau der Justizverfassung zu berücksichtigen. In einem Staat mit einer derartig kleinen territorialen Ausdehnung, gab er Beugnot gegenüber am 20. Dezember $1809 \mathrm{zu}$ bedenken, les circonstances de localités ont plus de poids et les exceptions sont plus faciles ${ }^{209}$.

Trotz der Eile, mit welcher Herzog von Bassano auf die Bereinigung der Düsseldorfer Gesetzentwürfe drängte, um die Einführung der Gerichtsverfassung zum 1.Januar 1810 zu ermöglichen, ließ sich Beugnot mit seiner Stellungnahme Zeit. Er gab seinem Vorgesetzten in Paris erst Bescheid, nachdem das französische Zivilrecht bereits in Kraft getreten war. Ob er auf diese Weise die überstürzte Umwälzung der Gerichtsverfassung verhindern wollte, bleibt Spekulation. Ohnehin ist zu bezweifeln, daß das Projekt in der Kürze der Zeit durchsetzbar gewesen wäre. Schon die Aufstellung dreifacher Kandidatenlisten wäre wohl bis Anfang Januar kaum machbar gewesen. In seinem Schreiben vom 6.Januar 1810 teilte Beugnot dem französischen MinisterStaatssekretär mit, er habe nach wie vor Bedenken gegen die vollständige Übernahme der französischen Justizverfassung im Großherzogtum. Er lehnte den unmodifizierten Transfer des französischen Rechtssystems zum einen ab, weil im Großherzogtum andere Ausgangsbedingungen herrschten als in Frankreich, und zum anderen, weil die geplante Rechtsreform in Frankreich erhebliche Veränderungen innerhalb der Justizverfassung des Nachbarn erwarten ließ und damit nur bedingt als Beispiel herangezogen werden konn$t^{210}$. Hinzu kam, daß Beugnot grundsätzlich evolutionäre Reformen gegenüber blockhaften Umwälzungen favorisierte.

J'appuie sur ce besoin de transition et en quelque sorte d'école, par l'expérience que j'obtiens chaque jour de la faiblesse des hommes de loi du grand-duché, erläuterte er Anfang Februar 1810. Ils sont si éloignés de ceux de France que l'on s'exposerait à plus d'un genre de danger si on en exigeait tout à coup les mêmes services: c'est déjà beaucoup que de porter nos institutions chez les peuples voisins, mais il reste toujours une chose difficile, c'est de former des hommes pour ces institutions, en attendant le moment où ils naîtront de ces institutions mêmes ${ }^{211}$.

Nachdem sich herausgestellt hatte, daß in Düsseldorf keine Lösung zu erwarten war, nahm der Minister-Staatssekretär mit dem französischen Justizminister Claude-Ambroise Regnier Kontakt auf und bat ihn darum, die ministerielle Dekretvorlage inhaltlich an das französische Modell anzupassen ${ }^{212}$. Der

209 Schreiben Nesselrodes an Beugnot vom 20. Dezember 1809, ibid.

210 Schreiben Beugnots an Maret vom 6. Januar 1810, ibid. Dem Schreiben lag der Brief Nesselrodes in Kopie bei.

211 Schreiben Beugnots an Maret vom 5. Februar 1810, ibid.

212 Schreiben Marets an den französischen Justizminister vom 17. Februar 1810, ibid. Beugnot hatte demgegenüber darum gebeten, einer Staatsratskommission das Projekt zur Begutachtung zukommen zu lassen. Siehe das Schreiben Beugnots an Maret vom 6. Januar 1810 , ibid. 
Entwurf aus Düsseldorf sei zu mangelhaft, argumentierte er, pour être présenté à Sa Majesté. Plusieurs des dispositions qu'il renferme sont tirées soit des lois du pays, soit de celles de Hollande ou du Royaume de Westphalie \& ne sont point suffisamment en harmonie avec le systême français qui doit seul régier le Grand-Duche ${ }^{213}$. Mit dem Rekurs auf den Pariser Justizminister wurde die baldige Reorganisation der Gerichte endgültig vereitelt. Regnier benötigte beinahe ein halbes Jahr, um den Auftrag des Minister-Staatssekretärs zu erledigen. Dabei machte er sich nicht die Mühe, die Arbeit der beiden Düsseldorfer Minister zu korrigieren. Vielmehr entwarf er eine völlig neue Dekretvorlage. Darin übertrug er die Gerichtsverfassung Frankreichs vollständig auf das Großherzogtum ${ }^{214}$. In der Folgezeit gerieten die Reformplanungen noch mehrfach ins Stocken, weil die französischen Regierungsbeamten in Paris durch andere Arbeiten zu stark in Anspruch genommen waren, um sich mit dem Thema zu befassen. Erst Napoleon sorgte im Winter 1811 dafür, daß die seit mehr als drei Jahren geplante Justizreform letztlich realisiert wurde. Während seines Besuchs in Düsseldorf Anfang November 1811 erklärte er, daß die französische Gerichtsverfassung in unmodifizierter Form zu Anfang des kommenden Jahres im Großherzogtum eingeführt werden sollte ${ }^{215}$. Am 17. Dezember 1811 lag ihm daraufhin der gewünschte Dekretentwurf zur Unterzeichnung vor ${ }^{216}$.

Als Napoleon am 17. Dezember 1811 für das Großherzogtum eine neue Gerichtsorganisation erließ, war die Justizverfassung gleichwohl bei weitem nicht mehr mit dem identisch, was Murat im März 1806 vorgefunden hatte. Während der mehrjährigen Planungsphase hatten zwar die meisten Justizbehörden ihre Arbeit in gewohnter Weise fortgesetzt. Doch hatten die Staatsbehörden zusammen mit anderen Reformen bereits an verschiedenen Stellen der Gerichtsordnung Modifikationen vorgenommen und hierdurch einzelne Reforminhalte vorweggenommen oder zumindest projektiert. Durch die Ausdehnung der preußischen Bezirksverfassung auf das gesamte Großherzogtum Berg im August 1806 und die Übernahme der französischen Gemeindeverfassung im Oktober 1807 beispielsweise war die Entflechtung von Verwaltung und Justiz auf Amts- bzw. Kommunalebene vorangetrieben worden. Mit Hilfe der Gemeindereform hatte die Regierung zudem die Verstaatlichung und Homogenisierung der Tribunale in Gang gebracht. Die Magistratsgerichte wurden aufgelöst. Ihre Zuständigkeiten gingen provisorisch auf die Amtsrichter über. Darüber hinaus war es schon im Rahmen der Gebietsreform Ende 1808 zur Neueinteilung von

213 Ibid.

214 Schreiben des französischen Justizministers an Maret vom 17. Juli 1810, AN, AA 36.

215 Notes dictées par Sa Majesté vom 2. November 1811, AN, AF IV 1253.

216 Gesetz-Bulletin, 2. Abtheilung, Nr. 52. Zu den Justizreformen siehe RoB, Regierungsakten des Großherzogtums Berg, S. 226f.; Francksen, Staatsrat, S.84f.; SchuBERT, Französisches Recht, S. 144f.; vgl. ferner Herman LoHAusen, Die obersten Zivilgerichte im Großherzogtum und im Generalgouvernement Berg von 1812 bis 1819, Köln u.a. 1995, S.21f.; NeLleN, Entwicklung, S.37f. 
Jurisdiktionsbezirken nach französischem Muster gekommen. Ferner wurden mit dem Abbau der tradierten Gesellschaftsstrukturen seit 1808 entscheidende Weichen für die Auflösung der Sondergerichtsbarkeiten, namentlich der von den Grundherren ausgeübten Patrimonialgerichtsbarkeit, gestellt ${ }^{217}$. Mit dem Transfer des französischen Zivilrechts wurde überdies die Einführung der französischen Gerichtsbehörden unumgänglich.

$\mathrm{Da}$ die Justizverfassung lange vor der eigentlichen Übernahme der französischen Gerichtsorganisation in Bewegung geriet, war insofern nicht unproblematisch, als sich hieraus beträchtliche Diskrepanzen und Entwicklungsunterschiede innerhalb der Rechtsordnung ergaben. Darüber hinaus kam es wiederholt zu Unstimmigkeiten und Kompetenzkonflikten. Die Richter der Ämter Beyenburg und Barmen etwa, die mit der Gerichtsbarkeit des aufgelösten Magistratsgerichts von Ronsdorf kollegial beauftragt wurden, machten sich ihre Befugnisse streitig, weil sich keiner von beiden das lukrative Amtsverhör entgehen lassen wollte ${ }^{218}$. Die Beyenburger Gerichtsschreiber Mosblech und Court gerieten über die geographische Ausdehnung ihrer Amtsbezirke aneinander. Court, der provisorisch für das aufgelöste Stadtgericht Rade vorm Wald übernommen worden war ${ }^{219}$, ging davon aus, da $B$ mit Bildung der neuen Munizipalitäten gleichzeitig neue Gerichtssprengel entstanden seien, zumal ihn Provinzialrat Théremin hierin unterstützte. Théremin meinte, die neuen Gerichtsbezirke entsprächen den Munizipalitäten, und entschied daher provisorisch, Courts Zuständigkeitsbereich erstrecke sich auf die gesamte Munizipalität Rade vorm Wald ${ }^{20}$. Mehrere Richter griffen auf die Zuständigkeiten von Kommunalbehörden über, weil die Regierung im Rahmen der Kommunalreform die Aufgabenbereiche der Verwaltungsbehörden von denen der Gerichte abgegrenzt hatte, ohne gleichzeitig die Zuständigkeitsbereiche der Justizbeamten genau zu definieren ${ }^{221}$. Diese Frage solite im Rahmen der Reorganisation

\footnotetext{
217 Vgl. Kap. IV, 2.3.1.

218 Schreiben des Richters Althaus an den Innenminister vom 22. Juli 1808, HStAD, GB, Nr. 4465.

219 Schreiben des Innenministers an den Provinzialrat vom 12. September 1809, HStAD, GB, Nr.4463I.

220 Schreiben des Provinzialrats Théremin an den Innenminister vom 28. Oktober 1808, ibid.

221 Beispielsweise zitierte das Essener Landgericht die Borbecker Ortsbeamten wegen einer Verwaltungsverfügung vor Gericht. Der Borbecker Maire Leimgardt hatte angeordnet, auf dem Land eines Kötters einen Graben auszuwerfen. Dem Gericht wurde daraufhin vom Innenminister am 22. März 1810 befohlen, sich alles ferneren Decretirens darin so wie bey ähnlichen Vorfällen, wo amtliche Verfügungen der Verwaltungsbehörden Statt haben, zu enthalten, vgl. HStAD, GB, Nr.10916. Demgegenuber griff der Angermunder Amtsrichter im Jahre 1809 in Belange der Munizipalverwaltung ein, als sich drei Einwohner hilfesuchend an ihn wandten, die in einem Ratinger Wirtshaus einem alten Mann das Haar abgeschnitten hatten und dafür von dem Ratinger Maire mit einer Geldstrafe bestraft worden waren. Sein widerrechtliches Vorgehen verteidigte der Richter in seinem Rechtfertigungsschreiben vom 3. Februar 1809 dabei mit seiner Unkenntnis der neuartigen Kompetenzverteilung zwischen Justiz und Verwaltung. Da ich nicht wußte, daß die
} 
von Justiz und Polizei geklärt werden. Für Verunsicherung sorgte die Regierung des weiteren, indem sie bei Einführung der französischen Munizipalverwaltung die Kumulierung von Munizipal- und Justizämtern duldete und damit die konsequente Durchsetzung der Trennung von Justiz und Verwaltung mehrfach selbst preisgab. In Lennep etwa kam Innenminister Nesselrode der Bitte der ehemaligen Magistratsmitglieder nach, das ehemalige Magistratsgericht der Stadt nicht nach Rade vorm Wald zu verlegen, sondern in Lennep aufrechtzuerhalten. Mit der Rechtsprechung beauftragte der Innenminister provisorisch den zweiten Beigeordneten ${ }^{222}$. Dem Lüdenscheider Maire Kerksig genehmigte er am 10. Oktober 1809, beim Lüdenscheider Landgericht weiterhin als Assessor tätig zu sein. Dabei berief er sich auf die Kollegialität des Landgerichts sowie die baldige Reorganisation der Justiz. Eigentlicher Grund war aber wohl, daß Kerksig an die Übernahme des Mairieamts die Bedingung knüpfte, sein Einkommen als Richter nicht zu verlieren ${ }^{223}$. Die Kollisionen zwischen Verwaltung und Justiz häuften sich in einem solchen Maß, daß Nesselrode im Herbst 1808 veranlaßte, ein eigenes Konvolut über die Grenzlinie zwischen den Gerichts-, Verwaltungs- und Polizeibehörden anzulegen 224 .

Bei der Bewältigung der Schwierigkeiten, die durch die Vorwegnahme einzelner Reforminhalte bei gleichzeitiger Beibehaltung der überlieferten Ordnung verursacht wurden, standen den Staatsbehörden nur palliative Mittel zur Verfügung. So hatten sie bei Kompetenzstreitigkeiten keine andere Wahl, als die Justizdiener durch Ermahnungen zur Mitarbeit und Kooperation anzuhal$\operatorname{ten}^{225}$. Fehlte es an den notwendigen institutionellen Voraussetzungen, mußten sie transitorische Maßnahmen ergreifen. Zum Beispiel war die Regierung bei Einführung des Code Napoléon gezwungen, den alten Gerichten provisorisch die Aufgaben der französischen Tribunale zu übertragen ${ }^{226}$. Die Aufga-

obere Justizbehörde über die Directionen in Fiscalien nichts zu befehlen hatte, so glaubte ich wirklich in meinem ganz reinen Sinne, daß das Verfahren der Direction, worin sie der Entscheidung der höhere Autoriät mit Thätlichkeiten vorschreitet [...] unerlaubt und der Justiz eingreifend seye, erklärte er, HStAD, GB, Nr. 12425.

222 Die Petenten hatten am 14. November 1807 geschrieben, sie fänden sich bey der gegenwertigen Verfaßung wohl und glücklich und wünschten keine andere Verwaltung, HStAD, GB, Nr. 4460 .

223 Schreiben des Präfekten Romberg an den Innenminister vom 30. September 1809; Bewilligungsschreiben des Innenministers an Romberg vom 10. Oktober 1809, HStAD, GB, Nr.4420II.

224 Dieses Konvolut befindet sich in der Akte HStAD, GB, Nr. 9878.

225 Die Beamten der Ämter Barmen und Beyenburg, Althaus und Holthausen, hielt Nesselrode nach einigem Hin und Her zur Zusammenarbeit an. Der Gerichtsschreiber Court wurde Ende Oktober 1808 an seinen ursprünglichen Amtsbezirk erinnert. Justizbehörden, die Angelegenheiten der Verwaltung usurpierten, wurden auf die Grenzen ihrer Zuständigkeiten aufmerksam gemacht. Vgl. das Schreiben des Innenministers an den Provinzialrat Théremin vom 6. September 1808, HStAD, GB, Nr. 4465, sowie das Schreiben des Innenministers an den Provinzialrat vom 31. Oktober 1808, HStAD, GB, Nr. 4463I.

226 Schreiben des Präfekten Romberg an die Unterpräfekten und Maires des Ruhrdepartements, abgedruckt in: PVRD 1810, 3. Januar, S.3f. 
ben der Friedensrichter wurden den Untergerichten anvertraut, das Oberappellationsgericht übernahm die Funktionen des Appellationsgerichts, und die Vormundschaftsangelegenheiten gingen an die Untergerichte.

Conditio sine qua non für die grundsätzliche Regelung der Probleme und Auseinandersetzungen im Bereich der Rechtsprechung war die seit 1808 in Aussicht gestellte, aber erst Mitte Dezember 1811 veranlaßte Neuordnung der Gerichtsverfassung nach französischem Muster ${ }^{227}$. Seitdem besaß die Gerichtsorganisation ein einheitliches Fundament und klare Vorschriften. Die bis zu diesem Zeitpunkt geltenden Überbrückungsmaßnahmen traten außer Kraft. Die im Justizwesen vorhandenen Struktur- und Entwicklungsunterschiede wurden definitiv beseitigt.

Mit dem Transfer der französischen Gerichtsverfassung ging erstens die Rechtsprechung im Großherzogtum nunmehr vollständig auf den Monarchen über. Die in privater bzw. intermediärer Hand liegenden jurisdiktionellen Institutionen, die das Gewaltmonopol der Krone im Gerichtswesen bis dahin eingeschränkt hatten, wurden endgültig aus dem Justizapparat verdrängt. Recht sprachen seither allein landesherrliche Gerichte. Alle Richter wurden künftig vom Großherzog auf Vorschlag des Justizministers ernannt. In einem Punkt blieb die Souveränität des Landesfürsten jedoch weiterhin eingeschränkt: in dem der Kassation. Nur die Bescheide der Friedensgerichte und Polizeitribunale wurden im Großherzogtum selbst kassiert. Zuständig hierfür war der oberste Gerichtshof des Großherzogtums, der Düsseldorfer Appellationshof. Über die Urteile der erstinstanzlichen Gerichte, der Assisenhöfe und des Appellationshofs entschied dagegen der Kassationshof in Paris $^{228}$. Daß die Kassationsfrage in dieser Form gelöst wurde, hatten Beugnot und Nesselrode in gewisser Weise mitzuverantworten. Sie waren während der Reformplanungen dafür eingetreten, die Urteile der Gerichte der ersten Instanz vom Appellationshof und die Urteile des Appellationshofs vom Souverän kassieren zu lassen, um zu verhindern, daß der Staatsrat, wie von diesem selbst beantragt, mit der Kassation betraut und damit politisch aufgewertet wurde. Offiziell begründeten sie ihr Veto damit, die Übertragung der Kassation auf den Staatsrat konterkariere die angestrebte Trennung von Verwaltung und Justiz und sei angesichts des geringen Umfangs des Landes zudem nicht gerechtfertigt. Daß ihr Vorschlag die Unabhängigkeit der Justiz grundsätzlich in Frage stellte, war für sie insofern kein Problem, als sie die Macht des Monarchen nach wie vor für unteilbar hielten. Der Prinz sei immer schon die Quelle sämtlicher Macht gewesen, erklärten sie, et peut continuer sans inconvéniens d'être regardé comme la source de tous les pouvoirs et comme préposé à les surveiller et à les réprimer également tous. Ohnehin erwarteten sie keine Verstöße seitens des Düsseldorfer Appellationshofs. Abweichungen der Mitglieder dieses Gerichts resserrés dans un cercle très-étroit 
[sic] et placés sous les yeux du gouvernement seien kaum zu befürchten, meinten $\operatorname{sie}^{229}$.

Im Rahmen der Justizreformen wurde zweitens die mit Hilfe der Verwaltungsreformen bereits vollzogene Abgrenzung der Administration von der Justiz durch die Trennung der Rechtsprechung von der Verwaltung ergänzt. Die gerichtlichen Functionen, hieß es in Artikel 4 der neuen Gerichtsverfassung, sind und bleiben allezeit von den administrativen getrennt, die Richter dürfen [...] auf keine Weise die Operationen der Verwaltungsstellen hemmen, oder über Administrationshandlungen sich eine Cognition anmaßen ${ }^{230}$. Es wurde eine von den Administrativbehörden unabhängige und einheitliche Gerichtsorganisation mit Berufsrichtern und den in Frankreich üblichen Instanzen geschaffen. Jeder Kanton besaß ein Friedensgericht. Für die Hauptorte der Arrondissements war jeweils ein Tribunal der ersten Instanz vorgesehen. In den Bezirken Elberfeld und Siegen befolgte man in Paris den Rat der beiden Düsseldorfer Minister und verzichtete auf die Einrichtung eines erstinstanzlichen Tribunals. Die Gerichte von Düsseldorf und Dillenburg waren für die beiden Arrondissements zuständig. In den Verwaltungszentren der Departements wurde jeweils ein Schwurgericht für die Strafjustiz eingerichtet. Es handelte sich hierbei nicht um einen ständigen Gerichtshof. Der Assisenhof versammelte sich vielmehr vierteljährlich. Oberstes Gericht des gesamten Großherzogtums war der Appellationshof in Düsseldorf ${ }^{231}$. In Anlehnung an Frankreich wurde im Großherzogtum ebenfalls ein Öffentliches Ministerium errichtet. Hierbei handelte es sich um ein Rechtspflegeinstitut, das man im Großherzogtum nicht kannte. Diese Behörde war zum einen für die Strafverfolgung und Durchführung der Klagen vor Gericht zuständig. Zum anderen übernahm sie Kontrollfunktionen gegenüber der Rechtsprechung. Sie überwachte den Geschäftsgang bei den Gerichten und beaufsichtigte das Justizpersonal. Die Bediensteten des Öffentlichen Ministeriums vertraten das Staatsoberhaupt vor Gericht und unterstanden dem Justizministerium. Leiter der neuen Behörde war ein Generalprokurator. An den einzelnen Gerichten des Großherzogtums wurde das Öffentliche Ministerium durch Prokuratoren und Substitute vertreten ${ }^{232}$.

\subsubsection{Rolle und Bedeutung der Rechtsprechung}

Während die großherzoglich-bergische Justiz durch die Einführung der französischen Gerichtsverfassung erstmals einheitliche und rationale Grundlagen erhielt, sah die Regierung in der Rechtsprechung keine unabhängige Staatsgewalt. Ansonsten hätte sie nicht an der Verquickung von Justiz- und Innenmi-

229 Observations des Ministres sur le travail de la Commission nommée pour préparer un Projet d'Organisation judiciaire dans le Grand-duché (September 1811), AN, AA 36. Ausführlich mit den Problemen der Kassation beschäftigen sich FrancKSEN, Staatsrat, S. 91f.; SCHUBERT, Französisches Recht, S.535f.

230 Gesetz-Bulletin, 2. Abtheilung, Nr. 52.

231 NeLLEN, Entwicklung, S.51f.

232 Gesetz-Bulletin, 2. Abtheilung, Nr. 52, Art. 68f.; NelleN, Entwicklung, S. 61f. 
nisterium weiterhin festgehalten. Ebensowenig hätte sie unmittelbar nach Erlaß der neuen Gerichtsverfassung einzelne jurisdiktionelle Kompetenzen der allgemeinen Rechtsprechung ausgegliedert. Im Rahmen der Neuordnung der Exekutive im März 1812 wurde der Düsseldorfer Staatsrat mit der Verwaltungsgerichtsbarkeit des Landes beauftragt. In Reaktion auf die Zunahme des Schmuggels und die vermeintliche Parteilichkeit von Richtern und Verwaltungsdienern in Schmuggelangelegenheiten entzog die Regierung den allgemeinen Gerichten Ende Januar 1812 die Zuständigkeit für Zolldelikte und übertrug diese einem eigens dafür ins Leben gerufenen Sondergericht, dem Spezialtribunal der Zölle. Dieses unterstand nicht dem Justiz-, sondern dem Finanzministerium. Seine Urteile mußten vom Staatsrat bestätigt werden, der somit in dieser Sache die Funktion des Kassationshofs wahrnahm ${ }^{233}$. Nach dem Ausbruch von Unruhen zu Beginn des Jahres 1813 gingen die Düsseldorfer Minister sogar soweit und setzten zur schnellen Bestrafung von Protestteilnehmern eine Militärkommission ein, obwohl nach französischem Recht Kriegsgerichte nur über Militärpersonen urteilen durften ${ }^{234}$. Mehrere Angeklagte wurden von dem Militärtribunal umgehend zum Tode verurteilt und zur Abschreckung öffentlich exekutiert, wie etwa bei der Konskriptionsziehung in Solingen. Cet exemple de sévérité, hoffte Nesselrode, fera une salutaire impression sur la majorité des habitans du Canton qui se trouveront réunis aujourd'hui à Solingen ${ }^{235}$. In den Folgetagen wurde gegen weitere Personen die Todesstrafe verhängt und das Urteil vollstreckt ${ }^{236}$.

Ein weiteres Indiz dafür, daß die Regierung die Justiz nicht als unabhängig betrachtete, liefern die wiederholten Anstrengungen des kaiserlichen Kommissars, die Rechtsprechung politisch in die Pflicht zu nehmen. Um etwa die zunehmende Ausweitung des Schmuggels und die damit einhergehende Eskalation der Konflikte zwischen Zolldienern und Einwohnern im Verlauf des Jahres 1812 in den Griff zu bekommen, nötigte Beugnot den Leiter des Öffentlichen Ministeriums, Generalprokurator Sethe, Anfang September 1812, die Prokuratoren der erstinstanzlichen Gerichte zur strengen Ahndung von Schleichhandelsdelikten anzuhalten. Einwohner, die in Kollisionen mit Zolldienern verwickelt waren, sollten künftig hart bestraft werden ${ }^{237}$.

233 Gesetz-Bulletin, 2. Abtheilung, Nr.89. Vgl. auch das Schreiben Roederers an Napoleon vom 27. Januar 1812, AN, AF IV 1853'.

234 Vgl. das Schreiben Nesselrodes an Roederer vom 28. Januar 1813, AN, 29 AP 39, sowie das Schreiben Beugnots an Roederer vom 4. Februar 1813, AN, 29 AP 38. Deshalb wurde die Militärkommission im General-Gouvernement auch für nicht kompetent erklärt. Darüber hinaus wurden Unregelmäßigkeiten während der Sitzung sowie das Fehlen einer Anklage-Akte bemängelt. Vgl. das Schreiben des Verteidigers Cremer vom 6. Dezember 1814, HStAD, GG, Nr. 1222.

235 Nesselrode in seinem Schreiben an Roederer vom 3. Februar 1813, AN, 29 AP 39.

236 Am 3., 4., 10. und 11. Februar 1813 wurden in Elberfeld und Solingen mehrere Insurgenten exekutiert. Schreiben Nesselrodes an Roederer vom 3., 5. und 11. Februar 1813, ibid.; Schreiben Beugnots an Roederer vom 13. Februar 1813, AN, 29 AP 38.

237 Schreiben Beugnots an Sethe vom 7.September 1812, ibid. 


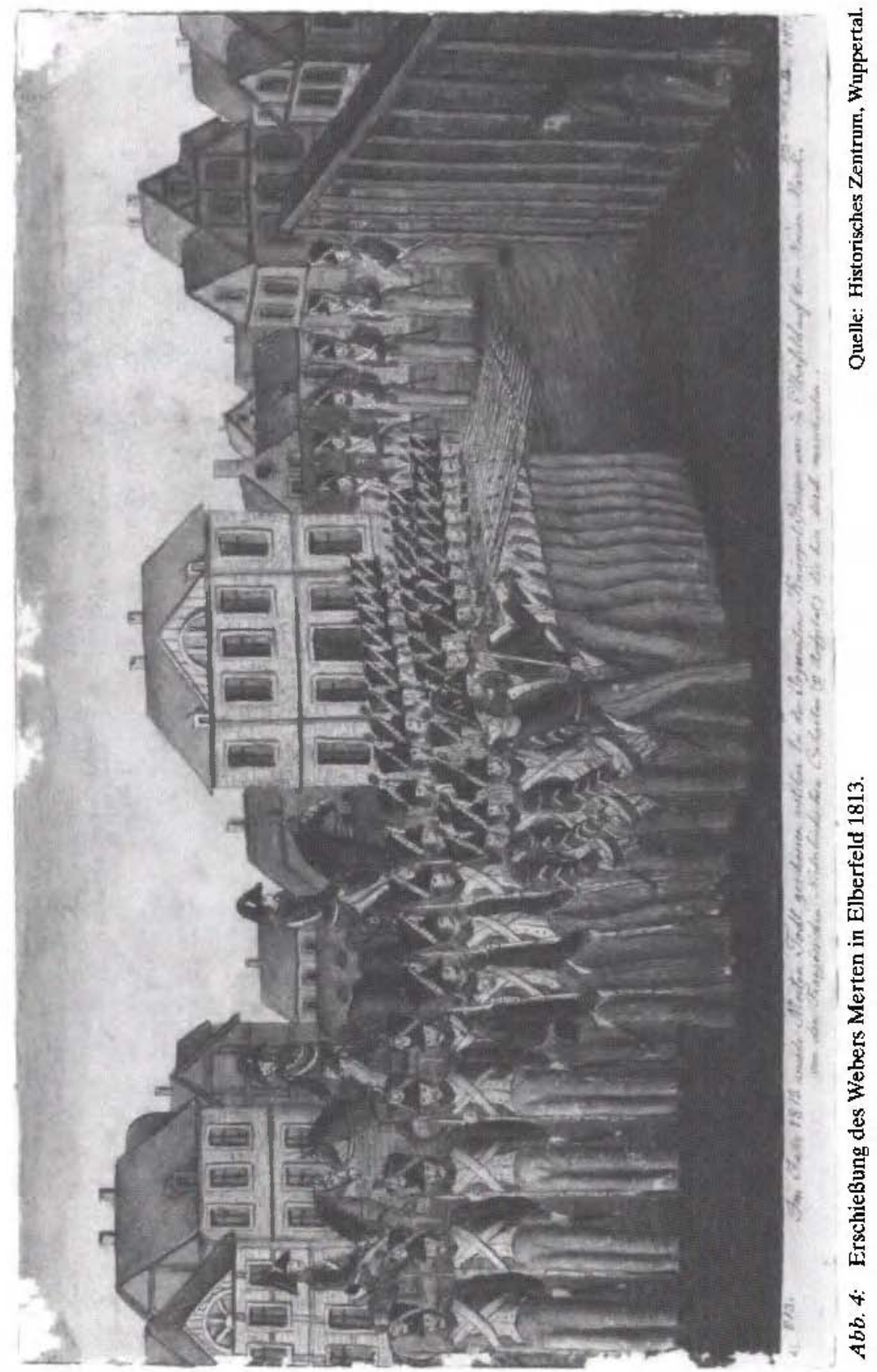


Anstoß zu der Intervention in die Rechtsprechung gaben Ausschreitungen zwischen Staat und Bevölkerung im Ortsteil Roth der Mairie Hörbach, im Siegdepartement gelegen. Am 27.August 1812 wollten Zollbedienstete in Roth Hausdurchsuchungen vornehmen, weil in der Gemeinde bisher angeblich kaum Salz und Tabak abgenommen worden waren. Die geplanten Visitationen der Douaniers sorgten in der Kommune für Aufruhr. Einwohner liefen zusammen und verhöhnten die Amtsträger. Nach dem Fall eines Schusses und der Verhaftung von zwei Personen eskalierte die Situation. Mit Stöcken, Hakken und Gabeln bewaffnete Bauern bewarfen die Repräsentanten der Staatsgewalt mit Steinen. Einer der Zolldiener verlor daraufhin die Kontrolle und schoß in die Menge. Zwei Bauern kamen hierbei zu Tode, einer wurde verwundet. Der Douanier wurde von der Gendarmerie verhaftet ${ }^{238}$.

Der kaiserliche Kommissar meinte, die Rechtsprechung trage an den Ausschreitungen in Roth eine gewisse Mitschuld. Im Unterschied zu seinem einheimischen Kollegen ${ }^{239}$ war er davon überzeugt, daß die Gerichte die Einwohner absichtlich nicht genügend bestraften und auf diese Weise indirekt für die seit Jahresbeginn zunehmenden Gewalttätigkeiten zwischen Zolldienern und Gemeindebewohnern bzw. den Mißerfolg der Zollgesetzgebung mitverantwortlich waren. Beugnot hielt es deshalb für notwendig, die Gerichte an ihre Pflichten gegenüber dem Staat zu erinnern. Einen Verstoß gegen die Gewaltenteilung oder einen Mißbrauch der Rechtsprechung sah er in seinem Lenkungsversuch nicht. Für ihn gehörte es zu den wesentlichen Funktionen der Rechtspflege, bei der Durchsetzung politischer Ziele Hilfe zu leisten, ja, in seinen Augen hatten die Justizdiener als staatliche Amtsträger die oberste Pflicht, den Willen des Landesherrn durchzusetzen. Er hielt es somit nur für legitim und notwendig, die Justizdiener an ihren politischen Auftrag zu erinnern.

Sethe duldete indessen nicht, daß Beugnot die Rechtsprechung politisch vereinnahmte. Seiner Meinung nach hatte der kaiserliche Kommissar keinerlei Befugnis zu einer solchen Intervention. Klagen über die Arbeit der Prokuratoren waren ihm zufolge nicht Sache des kaiserlichen Kommissars, sondern mußten an den Generalprokurator als dem eigentlichen Kontrollorgan der Gerichtsdiener direkt gerichtet werden. Die konkreten Angriffe Beugnots auf die Strafjustiz im Schmuggel hielt Sethe ebenfalls für unbegründet. Er behauptete, daß die Gerichtsdiener ihre Aufgaben in diesem Bereich ordnungsgemäß erfüllten. Den Auflauf in Roth versuchte er mit dem Argument zu bagatellisieren, die Ortseinwohner seien in erster Linie von Neugierde geleitet worden ${ }^{240}$.

238 Undatiertes Protokoll des Gendarmen Reichenau (28. August 1812), HStAW, Abt. 370 , GB, Nr. 828. Vgl. zu dem Vorfall in Hörbach auch Kap. III, 2.2.2.5.

239 Nach Auffassung des Innenministers beschränkte sich die Einstellung der Gerichte nicht à des sentimens aussi condamnables que le seraient la partialité pour les fraudeurs et la prévention contre les agens du gouvernement [sic] (Schreiben Nesselrodes an Beugnot vom 9. November 1810, HStAD, GB, Nr.6305).

240 Antwortschreiben Sethes an Beugnot vom 15. September 1812, AN, 29 AP 38. 
Der kaiserliche Kommissar ließ sich von Sethes Argumenten nicht überzeugen.

Il y a eu, teilte er Roederer Ende September 1812 mit, depuis trois mois vingt rassemblemens [sic], et que dans quelque-uns il s'est commis des meurtres: un seul a-t-il été poursuivi par les Procureurs Impériaux? Non. Voilà ce qui les accuse. [...] Il n'y a donc, schloß er daraus, à rechercher ni calomnie, ni calomniateurs, mais à faire autrement qu'on n'a fait jusqu'ici ${ }^{241}$.

Um das Vertrauen der Bevölkerung in die gerade erst eingeführte Rechtsordnung nicht zu gefährden und die Reformbemühungen der Regierung nicht zu diskreditieren, sah Beugnot allerdings von weiteren Interventionen $a b$ und akzeptierte damit faktisch die von Generalprokurator Sethe verfochtene Unabhängigkeit der Justiz. Im Fall Roth leitete er sogar die gerichtliche Verfolgung des von der Gendarmerie verhafteten Zolldieners Görzel ein, obwohl er eigentlich der Ansicht war, daß dieser von seiner Waffe legitimen Gebrauch gemacht hatte ${ }^{242}$.

Politische Motive waren es auch, die den kaiserlichen Kommissar nach den Unruhen Ende Januar bzw. Anfang Februar 1813 abermals dazu veranlaßten, sich in die Rechtsprechung einzumischen. Seiner Meinung nach erfüllten die Gerichte nicht die Funktionen, die ihnen im Rahmen der politischen Strafverfolgung zukamen. L'ordre judiciaire, pendant la période qui vient de s'écouler, kritisierte er im Sommer 1813, n'a pas eu seulement à s'occuper d'un grand nombre de causes ordinaires: il a eu à poursuivre des délits politiques [...]. Il faut le dire: l'ordre judiciaire n'a pas justifié dans ces circonstances les espérances qu'on en avait conçues ${ }^{243}$. An anderer Stelle meinte er: Tous conspiraient pour sauver les accusés de révolte ${ }^{244}$. Nur ein Teil der Justizdiener, rapprochés du Gouvernement, mieux traités, déjà distingués par ses faveurs, fiers d'une autorité dont l'exercice dépend davantage de leur libre arbitre, devaient être et se sont montrés dans la circonstance attachés au parti du Gouvernement ${ }^{245}$. Die übrigen hätten unter Einhaltung des verfassungsrechtlichen Rahmens für die Bevölkerung Partei ergriffen ${ }^{246}$.

Konkret machte Beugnot den Gerichten zwei Vorwürfe. Einerseits beschuldigte er sie, die Prozesse absichtlich hinauszuzögern, um auf diese Weise Freilassungen leichter erwirken zu können. Von 150 verhafteten Personen war seinen Aussagen zufolge im September 1813 noch niemand verurteilt worden ${ }^{247}$. Andererseits unterstellte der kaiserliche Kommissar den Gerichten Nachsicht bei der Verurteilung derjenigen, die während der Unruhen festgenommen wor-

241 Monatsbericht Beugnots an Roederer für August 1812, ibid.

242 Das teilte er Präfekt Schmitz in seinem Schreiben vom 28. September $1812 \mathrm{mit}$, HStAW, Abt. 370, Nr. 828.

243 Bericht Beugnots an Roederer vom 7. August 1813, AN, AF IV 1865.

244 Schreiben Beugnots an Roederer vom 10. Juli 1813, AN, AF IV 1840.

245 Ibid.

246 Ibid.

247 Bericht Beugnots an Roederer vom 7. August 1813, AN, AF IV 1865. 
den waren. Darüber hinaus warf er ihnen vor, die von den Aufständischen begangenen Vergehen bewußt zu verharmlosen. Seiner Meinung nach wurden die meisten Angeklagten unter dem Vorwand freigesprochen, sie hätten gemäß Art. 229 des Code d'Instruction kein vom Gesetz vorgesehenes Delikt begangen oder nach Art. 64 des Code pénal unter Zwang gehandelt. C'est voie une fois ouverte et bien élargie, kommentierte Beugnot diese Spruchpraxis, on y a marché avec un rare succès. Il n'y a pas encore un accusé marquant pour lequel on n'ait établi le fait de la contrainte, de sorte qu'au milieu de tant d'hommes contraints on ne peut pas savoir où s'est cachée la force qui les contraignait ${ }^{248}$.

Für besonders impertinent hielt der kaiserliche Kommissar den Freispruch des Gummersbacher Friedensrichters Pollmann. Auf Empfehlung des Generaladvokaten Gottfried von Sandt hatte der Düsseldorfer Appellationshof Pollmann am 1.April 1813 von jeglicher Strafe entbunden ${ }^{249}$. Nach Auffassung des Generaladvokaten war die Situation in Gummersbach so gravierend gewesen, daß Pollmann keine andere Wahl besessen hatte, als seine ehemaligen Funktionen als Vogt wiederaufzunehmen.

Considérant qu'il résulte, hatte Sandt am 27. März 1813 erklärt, de tout ce qui a été dit que, quoique le prévenu eut fait publier l'abolition des nouvelles institutions et le retablissement des anciennes, ce fait néanmoins ne peut lui être imputé, attendu qu'il y a été contraint par une force a laquelle [sic] il ne pouvait résister, et que d'ailleurs il n'a cédé à cette force que pour prévenir de plus grands malheurs, et pour gagner du temps de prendre les mesures que les circonstances exigeaient, et qu'en conséquence la conduite du prévenu ne présentait le caractère ni d'un crime ni d'un délit ${ }^{250}$.

Der kaiserliche Kommissar hielt die Behauptungen des Generaladvokaten für eine Frechheit. Seiner Meinung nach wurde der Umsturz der kaiserlichen Regierung auf diese Weise zu einem verdienstvollen Akt stilisiert bzw. zu einem nicht untersuchungswürdigen Vergehen minimalisiert ${ }^{251}$.

Beugnot führte die politische Unzuverlässigkeit der Gerichte hauptsächlich auf die schwache Kontrolle der Justiz zurück. Da es an einer festen Hand fehlte, erläuterte er Roederer Ende März 1813, qui tienne les juges dans le devoir et comprime leur disposition naturelle à s'echaper [sic] - womit er auf Sethe anspielte -, seien die Richter der Meinung, que tout le reste leur est soumis, et qu'ils ne sont soumis à personne. De là une affectation d'indépendance voisine de l'insolence ${ }^{252}$. Außerdem vertrat er nach wie vor den Standpunkt, die auf die Größe Frankreichs zugeschnittene Justizverfassung sei für das Großherzogtum Berg nicht geeignet. Das Gerichtswesen, wie es in Frankreich bestehe, wiederholte er Anfang August 1813, sei trop fort et [...] trop couteux pour le

248 Schreiben Beugnots an Roederer vom 10. Juli 1813, AN, AF IV 1840.

249 Urteil des Düsseldorfer Appellationshofs vom 1. April 1813, ibid. Zum Fall Pollmann siehe auch Kap. IV, 2.1.

250 Erklärung des Generaladvokaten Sandt vom 27. März 1813, AN, 29 AP 38.

251 Schreiben Beugnots an Roederer vom 5. April 1813, ibid.

252 Geheimbericht Beugnots an Roederer für März 1813, ibid. 
Grand-Duchêe 253 . Für die unbefriedigenden Leistungen der Justiz machte der kaiserliche Kommissar außerdem personelle Gründe verantwortlich. Einerseits hielt er die Besetzung der Schwurgerichte für schlecht. Die Laienrichter waren in seinen Augen schwach und borniert ${ }^{254}$. Andererseits lagen ihm zufolge die meisten Richterstellen in der Hand politisch unglaubwürdiger Personen, denn sie wurden von Preußen bekleidet, gens habiles à trouver le côté d'un instrument par où il peut devenir instrument de dommage 255 .

Innenminister Nesselrode stimmte seinem Kollegen in der Sache zu. Auch er meinte, im Rahmen der innenpolitischen Krisensituation hätte die Rechtsprechung anders ausfallen müssen, als es de facto geschehen sei ${ }^{256}$. Im Unterschied zu seinem französischen Kollegen war er aber davon überzeugt, daß die Justizdiener nicht mutwillig gehandelt hatten, sondern mit ihrem neuen Amt noch nicht hinlänglich vertraut waren.

Ich bin mehr und mehr davon überzeugt, verteidigte er das Gerichtspersonal, que nos jurisconsultes allemands accoutumés de prendre à la lettre chaque loi qu'on leur donne pour règle, enthousiasmés même de la force des lois qu'on vient de leur donner, ont conçu une opinion peut-être trop haute de leurs fonctions et que plusieurs d'entre'eux [...] manquent en partie de tact pour apprécier encore à sa juste valeur l'instrument dont on les a fait les dépositaires, ainsi que les modifications que les circonstances peuvent \& doivent apporter à ses loix, qui leur paraissent des règles de fer que rien ne devrait faire plier, enfin ils n'ont pas d'idée juste encore de ce qu'on appelle la haute police qui par fois et nommément dans des tems [sic] critiques exige des mesures extraordinaires ${ }^{257}$.

Nachdem Roederer von den Vorgängen Kenntnis erhalten hatte, erwog er im April 1813 kurzzeitig, die Gerichte des Großherzogtums in die Schranken $\mathrm{zu}$ weisen. Il peut etre nécessaire, äußerte er am 10. April 1813, de prendre quelques mesures afin d'empêcher que la conduite des magistrats ne devienne le motif ou l'excuse de l'insoumission des habitans [sic] ${ }^{258}$. Konkrete Maßnahmen ergriff der Kanzleichef indes nicht. Die Gerichte wurden von Zurechtweisungen und Bestrafungen verschont. Ihre Urteile blieben rechtskräftig. Entlassungen von Gerichtsdienern erfolgten ebensowenig.

Nur in einem Fall akzeptierten die Regierungsbeamten die Entscheidung der Richter nicht: in der Angelegenheit des Gummersbacher Friedensrichters Pollmann. Mit dem Argument, die Absolution Pollmanns habe Signalwirkung, erwirkte Roederer von Napoleon wenige Tage nach Freilassung des Friedensrichters einen erneuten Haftbefeh ${ }^{259}$. Gleichzeitig setzten sich die drei Mini-

253 Beugnot in seinem Schreiben an Roederer vom 7. August 1813, AN, AF IV 1865.

254 Ibid.

255 Beugnot in seinem Geheimbericht an Roederer für März 1813, AN, 29 AP 38.

256 Schreiben Nesselrodes an Roederer vom 6. April 1813, AN, 29 AP 39.

257 Nesselrode in seinem Schreiben an Roederer vom 4. Mai 1813, AN, AF IV 1834.

258 Schreiben Roederers an Napoleon vom 10. April 1813, AN, AF IV 1840.

259 Bericht Roederers an Napoleon vom 12. April 1813, AN, AF IV 1226. Nach seiner Freilassung hatten Nesselrode und Beugnot dem Friedensrichter befohlen, Düsseldorf 
ster des Großherzogtums mit der Frage auseinander, wie in der Angelegenheit weiter zu verfahren sei.

Niemand von ihnen bestritt hierbei, daß es sich um einen Straftatbestand handelte. Pollmann hatte in den Augen der drei Ressortleiter die Staatsgewalt angegriffen, zumal er auch nach Abzug der Aufständischen mehrere Tage lang weiterhin als Vogt amtierte. Pollmann hätte daher auch gemäß Artikel 87 des Strafgesetzbuches verurteilt werden müssen.

Effectivement, schrieb Innenminister Nesselrode am 6. April 1813 nach Paris, je ne puis m'empêcher de croire qu'il y avait délit dans l'abolition tentée de l'ordre actuel \& rétablissement de l'ancien; donc qu'il fallait mettre en jugement le prévenu de délit ${ }^{260}$. Allerdings gab er zu bedenken, que le dire des témoins lui est tellement favorable en constatant la manière dont il a été forcé à faire la proclamation en question, que la cour spéciale aurait difficilement pu s'empêcher de ne pas l'acquitter ${ }^{261}$.

Einig waren sich die Minister auch über das Versagen des von Sethe geleiteten Öffentlichen Ministeriums in dieser Angelegenheit. Aufgrund seines politischen Auftrags hätte dieses für die Verurteilung Pollmanns sorgen müssen, statt den Freispruch des Appellationshofes anzuerkennen und die Freilassung Pollmanns zu veranlassen.

C'est en jugeant, erklärte Beugnot am 6. April 1813, qu'on aurait examiné de quelle force, de quelle durée a été la contrainte exercée contre un juge de paix qui fait une proclamation, qui la fait a froid, qui la porte au curé pour la publier, qui assiste a [sic] la publication, qui en vertu de sa proclamation exerce des fonctions de bailli depuis que les brigands s'étaient retirés ${ }^{262}$.

Umstritten war unter den Verwaltungschefs demgegenüber die Frage, welche Maßnahmen konkret zu ergreifen waren. Zwar stellte keiner der Ministerialbeamten die verfassungsrechtlichen Rahmenbedingungen in Frage. Doch vertraten die Minister jenseits dieses Minimalkonsenses unterschiedliche Auffassungen darüber, wie weit die Regierung innerhalb der vom Gesetz vorgegebenen Grenzen im einzelnen gehen sollte und durfte. Innenminister Nesselrode sah die einzige Handhabe der Regierung darin, den Fall personalpolitisch zu lösen und Pollmann zu entlassen. Andere Optionen schieden für ihn wegen des Vorgehens des Öffentlichen Ministeriums von vornherein aus. Er betrachtete namentlich den Weg über die Kassation als blockiert ${ }^{263}$. Die beiden französischen Minister meinten ebenfalls, Pollmann müsse seines Amts enthoben werden. Sie hielten es aber zusätzlich für erforderlich, das Öffentliche Ministerium für seine vermeintliche Parteinahme zugunsten des Angeklagten poli-

nicht zu verlassen, und ihn überwachen lassen. Siehe das Schreiben Nesselrodes an Roederer vom 6. April 1813, AN, 29 AP 39.

260 Ibid.

261 Ibid.

262 Beugnot in seinem Schreiben an Roederer vom 6. April 1813, AN, 29 AP 38.

263 Innenminister Nesselrode in seinem Schreiben an Roederer vom 6. April 1813, AN, 29 AP 39. 
tisch zur Rechenschaft zu ziehen. Generalprokurator Sethe wurde von ihnen zu diesem Zweck im Mai nach Paris zitiert ${ }^{264}$. Gleichzeitig erkundigte sich Roederer im Pariser Justizministerium, si dans le cas même où la Cour d'appel aurait eu le droit de statuer sur les faits justificatifs, le Ministere public ne serait pas repréhensible d'avoir conclu au renvoi pur et simple de l'accusé, dans des circonstances extraordinaires, où la mollesse des Tribunaux pouvait enhardir les individus disposés à la révolte? ${ }^{265}$ Daneben drängten die beiden französischen Regierungsbeamten auf die Kassierung des Freispruchs durch den Kassationshof in Paris, denn ihnen zufolge hätte der Assisenhof und nicht das Appellationsgericht darüber entscheiden müssen, ob der Friedensrichter unter Zwang gehandelt hatte oder nicht ${ }^{266}$.

Daß Innenminister Nesselrode es im Gegensatz zu seinen französischen Kollegen bei der Entlassung Pollmanns belassen wollte, hing damit zusammen, daß er die Angelegenheit als Einzelfall betrachtete und damit keine weiteren Absichten verfolgte, als den Gummersbacher Friedensrichter für sein persönliches Fehlverhalten zur Verantwortung zu ziehen. Den französischen Regierungsbeamten hingegen ging es nur vordergründig um die Person Pollmanns. Für sie handelte es sich vielmehr um eine immanent politische Angelegenheit. Sie wollten die Justiz politisch an die Leine legen und sicherstellen, daß Recht künftig im Interesse des Landesherrn gesprochen wurde - nicht nur, um die aufrührerischen Elemente der Bevölkerung abzuschrecken, sondern auch und vor allem, um die großherzoglich-bergische Justiz, die sie gerade durch den Freispruch Pollmanns für kompromittiert hielten, in Paris zu rehabilitieren und weiteren Eingriffen von französischer Seite vorzubeugen ${ }^{267}$. Das Großherzogtum werde unmöglich einer Militärregierung entgehen, warnte Beugnot seinen Pariser Vorgesetzten Anfang April 1813, si les autorités civiles continuent de marcher aussi mal; si ce n'est pas un délit que le renversement du gouvernement impérial, si l'homme meme [sic] de l'Empereur établit cette doctrine et si le tribunal de l'Empereur l'accueille268.

Die französischen Minister konnten sich indessen nicht durchsetzen. Der Freispruch Pollmanns wurde nicht kassiert, weil der französische Generalprokurator Merlin darin keinen Verstoß gegen die geltenden Gesetze entdeckte. Das Urteil des Appellationshofs, erwiderte Generalprokurator Merlin Roederer am 8. Mai 1813, n'avait ni violé les règles de la compétence ni refusé le

264 Brief Roederers an den französischen Justizminister Regnier vom 14. April 1813, AN, AF IV 1840.

265 Ibid.

266 Schreiben des Generaladvokaten Sandt an Roederer vom 5.Juni 1813, AN, AF IV 1840; Schreiben Roederers an den französischen Justizminister Regnier vom 27. Mai 1813, AN, BB 317.

267 Falls die Kassation des Urteils ohne Einfluß auf das Schicksal Pollmanns blieb, sollte sie nach Roederer zumindest dazu dienen, die Rechtsprechung des Appellationshofs zu korrigieren, vgl. ibid.

268 Beugnot in seinem Schreiben an Roederer vom 6. April 1813, AN, 29 AP 38. 
caractère de crime à des faits qualifiés par la loi ${ }^{269}$. Deshalb könne es auch nicht kassiert werden. Die Frage, ob der Appellationshof gut oder schlecht geurteilt habe, gehörte ihm zufolge nicht zum Zuständigkeitsbereich des Kassationshofs ${ }^{270}$.

Roederer bemühte sich daraufhin vergeblich darum, den französischen Justizminister Ende Juni 1813 für die Kassation zu gewinnen. Regnier schloß sich der Entscheidung Merlins an, obwohl er Roederer anfangs grünes Licht gegeben hatte.

L'arrêt [...] laisse en effet, difficilement prise à la cassation, erklärte er. Wenn es im Gesetz heiße, qu'il n'y a ni crime ni délit, la compétence des chambres de mise en accusation semble résulter d'une manière de s'exprimer ainsi conçue, puisqu'elles sont spécialement autorisées à ordonner la mise en liberté des prévenus quand elles n'apperçoivent aucune trace d'un crime ou délit prévu par la lo $^{271}$. Der französische Justizminister wies in diesem Zusammenhang darauf hin, que cette sorte d'application du pouvoir attribué aux chambres d'accusations peut donner lieu à quelques abus mais d'un autre côté, on ne peut pas se dissimuler que la force majeure, la démence, sont souvent des circonstances tellement avérées et manifestes, qu'on ne saurait refuser aux chambres de mise en accusation de prendre l'initiative, en déclarant qu'il n'y ni crime ni délit ${ }^{272}$. Ebenso machte Regnier deutlich, daß es ihm in seiner Funktion als Leiter der Justiz vorbehalten war, d'examiner quelles mesures pourraient être prises pour concilier l'intérêt de la Société [...] avec l'exécution littérale de la loi pénale ${ }^{273}$. Doch im vorliegenden Fall hielt er es nicht für angebracht, d'insister à provoquer l'annullation de l'arrêt $t^{274}$.

Der Minister-Staatssekretär geriet durch die Absage der französischen Justizbeamten in Bedrängnis. Immerhin hatte er nach Pollmanns Freispruch Napoleon wiederholt auf die Schuld des Friedensrichters hingewiesen und den Appellationshof als nicht kompetent in dieser Angelegenheit bezeichnet wohl nicht zuletzt aus Angst, Napoleon könne von General Lemarois oder den seit Ausbruch der Unruhen in das Großherzogtum gesandten französischen Spionen von der Sache erfahren ${ }^{275}$. Nach dem Negativbescheid aus dem Pariser Justizministerium mußte der Minister-Staatssekretär gegenüber dem Kaiser die Aufrechterhaltung des Freispruchs im nachhinein doch noch rechtfertigen. Aus den Untersuchungsunterlagen gehe hervor, erklärte Roederer in diesem Zusammenhang, daß Pollmann unter Zwang gehandelt habe und daß

269 Das geht aus dem Schreiben Roederers an den französischen Justizminister vom 27. Mai 1813 hervor, AN, BB 317.

270 Ibid.

271 Der französische Justizminister Regnier in seinem Schreiben an Roederer vom 29. Juni 1813 , ibid.

272 Ibid.

273 Ibid.

274 Ibid.

275 So etwa in seinem Bericht an Napoleon vom 12. April 1813, AN, AF IV 1226. 
mit der Versammlung nur bezweckt worden sei, den Aufstand zu bekämpfen. Pollmann treffe daher allein der Vorwurf, schwach oder fehlerhaft gehandelt zu haben. J'estime en conséquence, schloß er daraus, que la cour d'appel n'a pa abusé de la confiance de V.M. ${ }^{276}$ Für den Gummersbacher Friedensrichter änderte sich hierdurch allerdings nichts. Er blieb auch weiterhin in Haft. Erst am 18. Oktober 1813 ordnete Beugnot seine Freilassung an 277.

Neben der Kassation des Freispruchs scheiterten die Minister auch mit ihren Bemühungen, die politische Weisungsgebundenheit des Öffentlichen Ministeriums durchzusetzen. Sethe weigerte sich, dem Druck der französischen Staatsbeamten nachzugeben. Als er während seines von Roederer und Beugnot erzwungenen zweimonatigen Aufenthalts in Paris schriftlich zu dem Thema Stellung nahm, pochte er darauf, daß das Öffentliche Ministerium unabhängig sei und Rechtsschutzfunktionen erfülle ${ }^{278}$. Le magistrat, meinte er, agissant toujours dans l'intéret de la Société est le vengeur de tous les troubles y apportés, mais en même tems [sic], et par la même raison le gardien de la liberté et de l'honneur des citoyens ${ }^{279}$. Zwar bestritt er nicht, que l'inaction du ministère public peut être commandée par des raisons d'état et des considérations politiques, und gestand sogar, mehrfach danach gehandelt zu haben ${ }^{280}$. Aber dies galt seiner Meinung nach nur für Verwaltungsangelegenheiten. In zivilen und kriminellen Angelegenheiten war das Öffentliche Ministerium für ihn ausschließlich dem Gesetz verpflichtet. Le magistrat spécialement chargé de faire respecter les lois, d'en surveiller l'exécution et de poursuivre leur infraction, pourrait-il lui même se rendre coupable d'une telle infraction? Le magistrat [...] pourroit-il requerir [sic] la mise en jugement ou la condamnation d'un individu s'il n'y a nulle trace d'un délit ou aucunes charges contre lui?, fragte er. Non, certainement, lautete seine Antwort, il est organe de la loi, \& impartial comme elle, il ne doit

276 Roederer in seinem an Napoleon gerichteten Projet de rapport sur les opérations des autorités judiciaires à l'occasion des troubles (Februar 1813), AN, 29 AP 37.

277 Verfügung des kaiserlichen Kommissars vom 18. Oktober 1813, HStAW, Abt. 370 GB, Nr. 940.

278 Der Generalpokurator verteidigte ebenfalls das Urteil des Appellationshofs im Fall Pollmann: la circonstance pouvait bien attirer sur le juge de paix une censure du Gouvernement pour cause de foiblesse ou d'imprudence [sic], legte er in seinem Exposé an Roederer aus dem Monat Mai 1813 dar, mais elle ne constituait pas un crime, AN, AF IV 1840.

279 Sethe in seinen an Roederer gerichteten Observations sur les attributions \& sur le caractère du Ministère public \& de celui du Procureur Général en particulier aus dem Monat Mai 1813, ibid.

280 Er habe das Verfahren gegen zwei Bedienstete des Finanzministeriums gestoppt, schrieb Sethe in diesem Zusammenhang, qui, l'année passée, avoient eu un démélé avec les percepteurs à la poste de Dusseldorf sur le payement d'un droit d'entrée, dit Sperrgeld, en portant l'affaire à la connoissance du ministre qui l'a terminé disciplinairement. Par les mếmes raisons je n'ai pas fait poursuivre les deux juges d'instruction du tribunal de Dusseldorf qui ayant eu rixe entre eux avoient exerçé des voies de fait l'un contre l'autre. Pour éviter le scandale qui résulteroit d'une telle poursuite, j'en ai réferé au ministre de la justice. Son Excellence a approuvé que la poursuite n'auroit pas lieu mais qu'une translocation des juges seroit proposée [sic] (vgl. ibid.). 
poursuivre que le coupable et non pas l'innocent. Avec le devoir des magistrats du ministère public, une conduite en qualité d'homme d'Etat ou politique, est à mon avis incompatible ${ }^{281}$. Was Sethe für das Öffentliche Ministerium reklamierte, verlangte er im übrigen für die Justiz insgesamt. Aussitôt qu'une affaire est portée en justice, erklärte er, elle doit être suivie et jugée dans les formes \& d'après les lois; tant le ministère public que les juges sont tenus de s'y conformer ${ }^{282}$.

Während der Leiter des Öffentlichen Ministeriums verhinderte, daß die Rechtsprechung zum verlängerten Arm der Exekutive wurde, besaß er umgekehrt keinerlei Mittel, um die Düsseldorfer Minister davon abzuhalten, Sonderregelungen zu treffen, die die bestehende Gerichtsverfassung unterliefen.

Vergeblich prangerte er an, qu'il a été établie une commission militaire, devant laquelle les insurgés seront mis en jugement. Je n'ai reçu aucune notice officielle sur l'établissement de ce Tribunal d'exception et des limites de sa competence; ni du décret impérial qui l'ordonne. [...] Or la subite apparition d'une commission militaire et l'entière exclusion des procureurs à l'égard des brigands pris par la Gendarmerie, doivent nécessairement surprendre les Procureurs et les rendre incertains s'ils pourront continuer d'exercer leur ministère et comment s'y prendre. Comme l'établissement d'une commission militaire, qui n'est pas fondée dans les lois organiques du Grand-Duché, ne peut être autorisée que par un décret impérial particulier, et que ce décret doit determiné si la commission militaire jugera tous les crimes commis par les réunions séditieuses, où jusqu'auquel point les Tribunaux ordinaires en connaitront; je prie Votre Excellence, de me faire communiquer une copie du décret impérial, qui ordonne l'établissement de la commission militaire, afin que je sache non seulement moi même [sic], si je puis exercer mon ministère, ou si je dois arrêter mes actions, mais que je puisse aussi, d'après sa teneur, instruire Mrs. les Procureurs près les Tribunaux ${ }^{283}$.

Noch weniger konnte Sethe verhindern, daß die beiden Düsseldorfer Minister nach dem Intermezzo von 1813 damit fortfuhren, außergerichtliche Verfahren anzustrengen und vermeintliche Unruhestörer durch Militärkommissionen verurteilen und anschließend hinrichten zu lassen. So ordnete Beugnot umgehend die Bildung einer Militärkommission an, nachdem in Gevelsberg eine Eskorte der Gendarmerie von bewaffneten Konskriptionsflüchtigen und Briganten Anfang Mai 1813 angegriffen worden war. Sie hatte einen Gefangenen von Düsseldorf nach Hagen bringen sollen, wo dieser von einer Spezialkommission verurteilt werden sollte. Ainsi tout individu, befahl Beutnot in diesem Zusammenhang, faisant partie des bandes armées contre l'ordre public dans le Grand Duché, encoure la peine de mort. [...] s'il peut être saisi, il doit être livré sur le champ à une commission militaire ${ }^{284}$. Er rechtfertigte diesen

281 Ibid.

282 Ibid.

283 Schreiben Sethes an den Innenminister vom 6. Februar 1813, ibid.

284 Schreiben Beugnots an den General Damas vom 3. Mai 1813, AN, 29 AP 38. 
Schritt mit der Vervielfachung der Delikte nach den Unruhen, vor allem aber mit der milden Entscheidungspraxis der Gerichte. Die daraufhin in Schwelm eingesetzte Militärkommission verurteilte den Häftling zum Tode. Die Vollstreckung des Urteils erfolgte am 6. Mai $1813^{285}$. Der Gastwirt Johann Christian Claudius Devaranne mußte sich am 26. Juli 1813 ebenfalls vor einer Militärkommission verantworten. Auch er wurde hierbei zum Tode verurteilt und hingerichtet. Devaranne galt als einer der Anführer und Drahtzieher während der Unruhen. Ihm wurde vorgeworfen, zusammen mit vier weiteren Personen Gewehre, Pulver, Blei sowie die Konskriptionslisten verlangt zu haben. Er war von den Gerichten zunächst freigesprochen worden ${ }^{286}$.

Der Befund der Analyse bleibt daher insgesamt zwiespältig. Aufgrund der Maßnahmen und Einflußversuche seitens der Regierung konnte sich die großherzoglich-bergische Justiz in französischer Zeit nicht zu einer Staatsgewalt im modernen Sinne entwickeln. Dennoch gab es Ansätze von Unabhängigkeit. Zum ersten hat das Verhalten der Regierung im Fall Pollmann deutlich gemacht, daß es unter den Ministern einen Minimalkonsens über die Grenzen der Exekutive im Bereich der Justiz gab, was im übrigen durchaus auf Ansätze von Verfassungsbewußtsein schließen läßt und gegen die These vom Scheinkonstitutionalismus spricht. Zum zweiten gelang es den Gerichten, die Deutungsmacht über die Klassifizierung normabweichenden Verhaltens für sich zu behaupten. Die Spruchpraxis der Justizbehörden gerade nach Beendigung der Unruhen läßt daran keinen Zweifel ${ }^{287}$. Zum dritten hat das erfolgreiche Vorgehen des Generalprokurators gegen die Versuche der Ministerialbürokratie, die Gerichte politisch zu vereinnahmen, bewiesen, daß die Rechtsprechung im Alltag durchaus Kontroll- und Rechtsschutzfunktionen gegenüber der Exekutive wahrzunehmen vermochte. Rechtsstaatliche Konturen besa $\beta$ die Justiz des Großherzogtums damit allemal.

\subsection{Wirtschafts- und Sozialordnung}

\subsubsection{Gesetzliche Neuerungen}

Neben den komplizierten Verhältnissen in den Bereichen Exekutive und Justiz gab auch die überlieferte Gesellschafts- und Wirtschaftsordnung der französischen Regierung genügend Anlaß zu Unzufriedenheit. Die im altständischen Ordnungsgefüge wurzelnden Privilegien und Schranken engten dort, wo sie noch bestanden, den Zugriff des Prinzen Murat auf seinen Untertanenverband erheblich ein und standen zu seinem Monopolanspruch auf die Herrschaftsausübung in eklatantem Widerspruch. Darüber hinaus konterkarierten

285 Bericht Beugnots an Roederer vom 3. und 7. Mai 1813, ibid.; Bericht Roederers an die Kaiserin vom 15. Mai 1813, AN, 29 AP 41.

286 HStAD, GG, Nr.1222.

287 Vgl. Andreas Würgler, Diffamierung und Kriminalisierung von »Devianz« in frühneuzeitlichen Konflikten. Für einen Dialog zwischen Protestforschung und Kriminalitätsgeschichte, in: HÄBERLEIN, Devianz, S. 317-347, hier S. 327. 
sie seine Reformbestrebungen in anderen Bereichen, erschwerten die Erfüllung der Pflichten, die dem Großherzogtum im Rahmen des napoleonischen Herrschaftssystems zukamen, und waren mit beträchtlichen finanziellen Einbußen verbunden.

Trotz dieser problematischen Tatbestände zeigte die französische Regierung in den ersten Monaten ihrer Amtszeit nur begrenztes Interesse an der Modifizierung der vorgefundenen Strukturen. Nur gelegentlich nahm sie Veränderungen darin vor. So veranlaßte Murat etwa im Spätsommer 1806 die bereits erläuterte Aufhebung der Grundsteuerbefreiungen, für welche sich Finanzminister Agar schon im Frühjahr 1806 und parallel zu seinen Bemühungen, die Stände zu reformieren, stark gemacht hatte. Außerdem führte er den Stempel ein und beseitigte alle Sonderrechte im Zollwesen durch die Einführung eines allgemeinen Zolltarifs ${ }^{288}$. Abgesehen von den steuerrechtlichen Veränderungen wurden geburtsständische Zugangskriterien bei der Vergabe öffentlicher Ämter weitgehend abgeschafft ${ }^{289}$ und am 9. Juni 1807 die Konskription eingeführt. Demnach waren alle im Großherzogtum ansässigen Männer im Alter von 20 bis 25 Jahren, auch die Söhne jüdischer Familien, künftig zur Absolvierung eines achtjährigen Militärdienstes verpflichtet ${ }^{290}$. Verschiedene Bevölkerungsgruppen waren indes von der Verpflichtung zum Dienst an der Waffe befreit. Hierzu zählten Adelige, die im Landtag stimmfähig waren, öffentliche Beamte, Geistliche, Lehrer öffentlicher Schulen, bedeutende Künstler und Fabrikanten, die Söhne der höheren Beamten sowie die von Witwen, sofern diese keine weiteren männlichen Nachkommen hatten ${ }^{291}$.

Die vereinzelten Initiativen der Regierung hingen unter anderem damit zusammen, daß es für Reformen nur begrenzten Handlungsspielraum gab. Einerseits waren Veränderungen im überlieferten Gesellschaftsgefüge wegen der Schaffung eines neuen Erbadels ${ }^{292}$ wie auch der fortdauernden Privilegierung der mediatisierten Fürsten gerade im fiskalischen Bereich nicht nur äuBerst problematisch, sondern in Paris auch nur schwer durchsetzbar. Andererseits mußte auf die Interessen der Bevölkerung Rücksicht genommen werden, denn bei der Herrschaftsausübung und der Durchsetzung von Reformen war man maßgeblich auf die Unterstützung und Mitarbeit der Einwohner ange-

288 Vgl. die Verfügung Joachim Murats zur Einfuihrung des Stempels vom 28. März 1807, AN, 29 AP 34, ferner die Verfügung des Großherzogs zur Einführung eines einheitlichen Zolltarifs im Großherzogtum vom 8. September 1807, ADD, Fonds Mosbourg, 100 MI 115 R2.

${ }^{289}$ Siehe Kap. III, 1.1., 2.1.

290 Landesherrliche Verfügung zur Einführung der Konskriptionspflicht vom 9. Juni 1807, §2, AN, 29 AP 34; siehe ferner Schmid,, Grand-Duché, S. 59f.; Bernd DreHER, Großherzoglich-bergische Truppen 1806-1813, in: Ders., Engelbrecht, Das Herzogtum Berg, S. 53-57, hier S. 54; KandIL, Sozialer Protest, S. 56.

291 Landesherrliche Verfügung zur Einführung der Konskriptionspflicht vom 9.Juni 1807, \$2, AN, 29 AP 34.

292 Siehe Tulard, Napoléon ou le mythe, S.325f.; Louis Bergeron, L'Épisode napoléonien. I. Aspects intérieurs 1799-1815, Paris 1972, S. $79 f$. 
wiesen. Der Hauptgrund für die Zurückhaltung der Regierung ist aber wohl in der landesherrlichen Reformpolitik selbst zu suchen. Diese richtete sich nach dem anfänglichen Scheitern der Verfassungspläne zuvörderst auf den Aufbau einer leistungsfähigen Verwaltung. Die Umgestaltung der altständischen Sozial- und Wirtschaftsstrukturen war dagegen von zweitrangiger Bedeutung: Gegenstand von Reformen wurden Elemente der Gesellschafts- und Wirtschaftsverfassung nur mittelbar im Rahmen anderer Reformprojekte oder wenn es um die Durchsetzung machtpolitischer Ambitionen ging.

Mit Hilfe der Konskriptionspflicht zum Beispiel wollte die Regierung die bisherigen Schwierigkeiten bei der Rekrutierung von Waffenträgern überwinden und die Bildung des Heeres sicherstellen, das sie im Rahmen des Rheinbundes aufzubieten hatte ${ }^{293}$. Die Beteiligung aller Einwohner an den öffentlichen Lasten diente demgegenüber dazu, die finanziellen Ressourcen des Großherzogtums für die französiche Herrschaft zu mobilisieren. Bestes Beispiel dafür war die Beseitigung der Steuerbegünstigungen im Grundsteuerbereich. Als Agar den Anstoß hierzu gab, ging es ihm hauptsächlich darum, die Einkünfte des Prinzen zu mehren. Nicht zuletzt deshalb behandelte er das Thema in seiner Denkschrift aus dem Monat März 1806 unter der Rubrik »Finanzen ${ }^{294}$. Allerdings machte sich Agar hierbei zunutze, daß die Besteuerung sämtlicher Einwohner einem Wunsch entgegenkam, der in der einheimischen Bevölkerung schon seit längerem gehegt wurde ${ }^{295}$. Außerdem verwies er programmatisch auf den Gleichheitsgrundsatz. Ce privilege féodal, kritisierte er die fiskalische Begüngstigung einzelner Einwohnerkreise, est aussi contraire da l'équité qu'aux régles d'une bonne administration ${ }^{296}$.

Während die Regierung mit ihren Eingriffen in die tradierten Ordnungsstrukturen keinem vorgefaßten Reformplan folgte, sondern konkrete Bedürfnisse und Interessen zu erfüllen suchte, ist nicht zu leugnen, daß sowohl die Abschaffung der Steuervorteile, die einzelne Bevölkerungsgruppen genossen, wie auch die Einführung neuer Rekrutierungskriterien und die Verpflichtung

293 Schmidt, Grand-Duché, S. 56f.; DrehER, Truppen, S.53f.

$294 \mathrm{Vgl}$. das undatierte Tableau de l'administration civile et judiciaire des duchés de Clèves et de Berg sous le régime prussien (März 1806), angefertigt von Agar, AN, AF IV 1225. Wie begrenzt der Reformwille der Landesherrschaft war, zeigt sich auch daran, daß diese die Politik ihrer Vorgängerregierungen fortsetzte, fehlende Einnahmen durch die Erhebung außergewöhnlicher Steuern zu mobilisieren, als die Steuereinnahmen nicht mehr dazu ausreichten, den besonders durch wachsende Militärausgaben aufgeblähten Staatshaushalt zu decken. Vgl. AN, AF IV* 460.

295 Karl Freiherr voN AYx, Einige Ideen über die Erreichung eines richtigen und genauen Abgaben-Systems, in Betreff der Grundsteuer im Herzogthum Berg, Düsseldorf 1806, passim. Der Freiherr polemisierte in dieser Reformschrift, da $B$ es noch immer zahlreiche fiskalische Befreiungen gebe und die Steuer ungerecht auf die Bevölkerung verteilt werde. Ferner erfolge die Steuererhebung unverändert nach einer uneinheitlichen Katasterordnung und mit Hilfe nicht aktualisierter Heberollen. Siehe ferner SCHMIDT, Grand-Duché, S.287f.

296 Undatiertes Tableau de l'administration civile et judiciaire des duchés de Clèves et de Berg sous le régime prussien (März 1806), angefertigt von Agar, AN, AF IV 1225. 
zum Dienst an der Waffe langfristig beträchtliche Auswirkungen auf die Verfassungsstrukturen des Großherzogtums hatten. Sie leisteten der Gleichstellung der Bevölkerung erheblichen Vorschub, wenn auch primär durch die Auferlegung gleicher Pflichten gegenüber dem Staat. Insofern reichten sie weit über die egalisierenden Einzelmaßnahmen der Vorläuferregierungen hinaus ${ }^{297}$.

Mit dem 1807 gefaßten Entschluß des französischen Monarchen, im Großherzogtum Berg das französische Zivilrecht einzuführen, bahnte sich in der französischen Reformpolitik ein Kurswechsel an ${ }^{298}$. Die geplante Rezeption des auf den spezifischen Verhältnissen Frankreichs beruhenden Zivilgesetzbuches machte es erforderlich, auch die Strukturen in Wirtschaft und Gesellschaft auf das sozialrevolutionäre Modell Frankreichs zuzuschneiden. Der bis dahin willkürlich und unsystematisch verlaufende Reformprozeß erhielt erstmals eine klare Richtung und ein festes Ziel. Allerdings kümmerten sich die Düsseldorfer Regierungsbehörden in der Anfangszeit nicht darum, konkrete Planungen zur Rezeption des französischen Zivilgesetzbuches in die Wege zu leiten. Reformplanungen kamen erst in Gang, nachdem sich Murat im Januar 1808 dazu entschieden hatte, bis zur Besitzergreifung der hinzugewonnenen Gebiete am 1.März 1808 das Großherzogtum Berg dem französisch-westfälischen Modell strukturell anzugleichen. Mit seinem Reformprojekt bestätigte der Prinz nicht nur die ein Jahr zuvor getroffene Richtungsentscheidung. Er verlieh seinem Vorhaben auch neue Qualität und Dynamik. Die Veränderungen, die er hierbei plante, gingen nämlich weit über den zivilrechtlichen Bereich hinaus und waren so tiefgreifend, daß dadurch die altständische Ordnung letztlich im Kern gesprengt wurde. Als Bestandteil eines Konstitutionsvorhabens erfüllten die Reformen überdies nicht mehr nur machtpolitische, sondern erstmals genuin verfassungspolitische Funktionen.

Finanzminister Agar beauftragte Anfang Februar 1808 eine Staatsratskommission damit, die Einführung des französischen Zivilrechts vorzubereiten und entsprechende Gesetzentwürfe auszuarbeiten. Obwohl die Staatsräte umgehend mit dieser Aufgabe begonnen ${ }^{299}$, wurde auch dieses Reformwerk während der Regentschaft des Prinzen nicht mehr verwirklicht. Mit dem Aufschub der Verfassungsgebung bzw. dem Ausbleiben einer geschriebenen Konstitu-

297 Im Herzogtum Berg und im ehemaligen Hochstift Münster wurden bei außergewöhnlichen Steuerausschreibungen neben den gesetzlich zur Zahlung Verpflichteten auch all diejenigen, die normalerweise keine Steuern zahlten, zur Aufbringung der Steuern herangezogen. Siehe Lenzen, Beyträge, Bd. 1, S. 87; Heinz REIF, Umbruchserfahrung und Konflikt. Adel und Bauern im Münsterland, in: Berding, Ullmann, Revolution und Restauration, S. 226-257, hier S.229.

298 Vgl. das Schreiben Joachim Murats an Agar vom 13. Mai 1807, ADD, Fonds Mosbourg, 100 MI 114 R2, sowie das Schreiben Agars an Joachim Murat vom 2. April 1807, ADD, Fonds Mosbourg, 100 MI 118; ferner RoB, Regierungsakten des Großherzogtums Berg, S. 170f.

299 Vgl. ibid., S.172f. 
tionsakte entfiel der konstitutionelle Rahmen, an den die Durchführung der Reformen gekoppelt war. Auf den Fortgang der Reformarbeit hatte dies insofern Einfluß, als die beabsichtigten Umwälzungen nicht mehr, wie geplant, geschlossen und mit den entsprechenden konstitutionellen Garantien durchgesetzt, sondern wie bisher auf dem Weg der Einzelreform und somit entsprechend langsamer weiterverfolgt wurden. Weitere Verzögerungen ergaben sich ferner aus der Notwendigkeit zur Integration der im Januar 1808 hinzugewonnenen Gebiete. Die Eingliederung der neuen Landesteile kostete die Regierung so viel Aufwand und Zeit, daß die von Murat seit Januar 1808 geplanten Eingriffe in das altständische Ordnungsgefüge im Stadium der planerischen Vorarbeit stecken blieben. Agars Aussagen zufolge war die Ausarbeitung der Gesetzentwürfe hingegen so weit gediehen, daß ihre Veröffentlichung möglich gewesen wäre ${ }^{300}$. Durch den Thronwechsel kamen die Planungen schließlich ganz zum Erliegen.

Die einzige noch unter Murats Herrschaft vorgenommene Neuerung war die am 22. Juli 1808 verordnete Abschaffung sämtlicher Abgaben, Tribute und Schutzgelder, welche die Juden bis dahin an die Domänenkassen zu entrichten hatten ${ }^{301}$. Diese Maßnahme hing mit den von Murat vorgenommenen Neuerungen im fiskalischen und militärischen Bereich in der Anfangszeit seiner Herrschaft zusammen und war auch gemeinsam mit diesen in Angriff genommen worden. Sie stand nicht mit den Planungen zum Transfer des französischen Zivilrechts in Verbindung. Offiziell zeichneten die beiden Düsseldorfer Minister für den Beschluß verantwortlich. Treibende Kraft in der Angelegenheit war aber wohl Finanzminister Agar, der großes Interesse daran hatte, die Sonderstellung der jüdischen Minderheit zu beseitigen. Er war es, der den Anstoß dazu gab, die Situation der jüdischen Minderheit im Großherzogtum überhaupt in den Blick zu nehmen.

Am 6.Juni 1807 forderte er sämtliche Provinzialräte des Großherzogtums dazu auf, ihm über die Lage der in ihrem Verwaltungsbezirk wohnenden Juden Bericht zu erstatten, weil es nun nicht schicklich seyn würde, dergleichen aus finstern Jahrhunderten herkommenden Einrichtungen ferner bestehen $z u$ lassen, seine Kayserliche Königliche Hoheit auch wünschen müssen, daß in allen Staaten eine gleichförmige Behandlung, der darin wohnenden, sowohl als darin Verkehr treibenden Juden eingeführt werde, und daß diese Einrichtung sich, ohne Huldigung irgend eines Vorurtheils, auf ächte Grundsätze der Staatswirthschaft, und auf das allgemeine Wohl aller Landeseinwohner erstrecke ${ }^{302}$.

300 Notes d'Agar, Comte de Mosbourg, sur les rapports de Beugnot à l'Empereur et à Gaudin (1808), AN, 31 AP 44.

301 General-Verordnung der Minister vom 22. Juli 1808, HStAD, GB, Nr.10888; teilweise abgedruckt in: RoB, Regierungsakten des Großherzogtums Berg, S.34.

302 So Agar in seinem Schreiben an die Provinzialräte vom 6.Juni 1807, HStAD, GB, Nr.10888. Auffällig an den Berichten der Provinzialräte sei, kommentierte der Verwaltungsrat Lenzen in seinem undatierten Rapport sur l'etat des Juifs du grand Duché de Berg [sic] an den Finanzminister (1807/1808), daß man die Juden dort am stärksten fürchte, wo 
Auf das Engagement des Finanzministers war ebenfalls zurückzuführen, daß die Frage der jüdischen Minderheit noch so kurz vor dem Regierungswechsel geklärt wurde.

Vieles spricht für die These, daß sich der Finanzminister in dieser Angelegenheit nicht ausschließlich von »ehrenvollen« Motiven leiten ließ, wie Klaus Rob annimmt ${ }^{303}$, sondern hierbei auch und vor allem utilitaristische Ziele verfolgte. Agar ging es darum, Probleme und Unstimmigkeiten aus dem Weg zu räumen, die sich daraus ergaben, daß die jüdischen Einwohner fiskalisch und militärisch ebenso in die Pflicht genommen wurden wie die übrigen Untertanen $^{304}$. Agar wollte - mit anderen Worten - klare Rechtsverhältnisse schaffen und die jüdischen Einwohner als steuerzahlende und wehrpflichtige Untertanen vollständig in den Staatsverband eingliedern. Für die Stichhaltigkeit dieser Annahme spricht einmal, daß der Finanzminister die Umfrage zur Erfassung der Lage der jüdischen Bevölkerung nur wenige Tage vor Einführung der Konskriptionspflicht in die Wege leitete. Dafür spricht ebenfalls der eigentliche Inhalt der Verfügung. Da die jüdischen Einwohner des Landes sowohl dem Wehrdienst als auch den öffentlichen Abgaben unterworfen seien, hieß es darin einleitend, seien von nun an alle bisher von den Juden an die Domänenkasse entrichteten Abgaben, wie Tribute, Schutzgelder und Heiratsabgaben, abgeschafft. Die Rentmeister der Domänen sollten keine weiteren Anforderungen an die in ihrem Verwaltungsbezirk wohnenden jüdischen Familien stellen ${ }^{305}$. Dies betraf sowohl rückständige als auch laufende Abgaben. Der emanzipative Gehalt der Verfügung rückte dagegen in den Hintergrund. Dem Erlaß der Minister war nur zu entnehmen, der Großherzog habe mehrmals seine Absicht zu erkennen gegeben, den Juden allmählich die nämlichen Rechte und Freiheiten zu verleihen, die die übrigen Bewohner des Großherzogtums genossen ${ }^{306}$. Genauere Bestimmungen hinsichtlich der künftigen Rechtsstellung der jüdischen Untertanen enthielt die Verfügung nicht. Nur vage stellten Agar und Nesselrode ein Reglement über die Rechte und Verbindlichkeiten der jüdischen Unterthanen in Aussicht ${ }^{307}$. Ein Ende Okto-

sie am meisten unterdrückt worden seien. Er schloß daraus, que l'immoralité des Juifs dans leurs rapports aux Chrétiens est en proportion avec l'état de leur oppression; et on en feroit la juste conséquence, qu'il n'y aura pas un meilleur moyen d'annoblir [sic] la nation juife, que d'accorder à elle les droits civils sans aucune restriction. Vgl. HStAD, GB, Nr.171.

303 Roв, Regierungsakten des Großherzogtums Berg, S. 12.

304 In der ehemaligen Unterherrschaft Hardenberg beispielsweise weigerten sich die jüdischen Einwohner, der Freifrau von Wendt die bisherigen Abgaben zu entrichten, weil sie die Auffassung vertraten, daß sie bei den Staatsaufgaben und Lasten den übrigen Einwohnern gleichgestellt und ihnen die persönlichen und staatsbürgerlichen Rechte erteilt worden waren. Vgl. die Gesuche der jüdischen Einwohner im ehemaligen Amt Hardenberg an den Innenminister vom 24. August 1807 und 4. Dezember 1807, HStAD, GB, Nr. 4293.

305 Zit. nach RoB, Regierungsakten des Großherzogtums Berg, S.34; vgl. HStAD, GB, Nr. 10888.

306 RoB, Regierungsakten des Großherzogtums Berg, S.34.

307 General-Verordnung der Minister vom 22. Juli 1808, HStAD, GB, 10888. 
ber 1808 vom Düsseldorfer Staatsrat vorgelegter Dekretentwurf zur rechtlichen Gleichstellung der Juden wurde nicht mehr realisiert ${ }^{308}$.

Dennoch ist nicht zu leugnen, daß von der Neuregelung der Lebensverhältnisse der jüdischen Einwohnerschaft wichtige modernisierende Impulse ausgingen. Zum einen implizierte die Beseitigung der in der altständischen Gesellschaft wurzelnden Abgaben das Recht auf Niederlassungsfreiheit, wenn auch nur für die einheimischen Juden. Ausländische Juden benötigten nach wie vor eine Genehmigung, um sich niederlassen zu können. Zum anderen wurde die Gleichstellung der jüdischen Einwohner mit der übrigen Bevölkerung und mithin deren Integration in den großherzoglich-bergischen Untertanenverband gefördert, indem die Juden, zumindest was ihre Pflichten gegenüber dem Staat betraf, wie die übrigen Einwohner behandelt wurden.

Als Beugnot im Sommer 1808 sein neues Amt in Düsseldorf antrat, kümmerte er sich zunächst nicht um die Weiterführung der von Murat zu Anfang des Jahres geplanten Reformen, sondern konzentrierte sich darauf, die landesherrlichen Einkünfte sicherzustellen ${ }^{309}$. Er befürchtete nämlich, daß Umwälzungen zu finanziellen Einbußen führen könnten ${ }^{310}$. Der Reformprozeß wurde erst wieder angekurbelt, nachdem Napoleon im September 1808 von seinem Statthalter ein Organisationsdekret für das Großherzogtum eingefordert und in diesem Zusammenhang seine Absicht bekanntgegeben hatte, das Großherzogtum Berg nach französischem Beispiel zu gestalten ${ }^{311}$.

Dreh- und Angelpunkt der daraufhin unternommenen Reformanstrengungen war und blieb die Zivilrechtsreform. Aus ihr ergaben sich Ziel und Inhalt der Umwälzungen im Wirtschafts- und Gesellschaftsbereich, nämlich die Einführung einer Ordnung, in der jeder über sein Eigentum frei verfügen konnte, gleiche bürgerliche Rechte genoß und persönlich frei war. Durch die Zivilrechtsreform wurde in gewisser Weise auch der Verlauf der Reform selbst vorgegeben. Da nämlich die Sozial- und Wirtschaftsverfassung des Großherzogtums Berg trotz nicht zu verkennender Erosionserscheinungen und vereinzelter Reformansätze grosso modo immer noch auf altständisch-korporativem Fundament beruhte und das französische Zivilrecht demnach etwas voraussetzte, was es im Großherzogtum noch nicht gab, hielten die Staatsbehörden es für notwendig, im Vorfeld der Zivilrechtsreform verschiedene Weichen zu stellen.

Als eine der wesentlichen Voraussetzungen für die Rezeption des Code $\mathrm{Na}$ poléon betrachteten die Reformer die Auflösung der in der ländlichen Sozialverfassung wurzelnden feudalrechtlichen Bindungen ${ }^{312}$. Die Verknüpfung von

308 Dekretentwurf des Düsseldorfer Staatsrats vom 28. Oktober 1808, HStAD, GB, Nr. 171.

309 Die Regelung der öffentlichen Einkünfte sei stets, schrieb Beugnot in seinem Antwortschreiben an den Pariser Finanzminister Gaudin vom 7.September 1808, la premiere chose dont on doive s'occuper. Vgl. AN, AF IV 1842.

310 Ibid.

311 Anweisung Napoleons an Gaudin vom 11. September 1808, AN, AF IV 1886 A.

$312 \mathrm{Vgl}$. zu diesem Sachverhalt RoB, Regierungsakten des Großherzogtums Berg, S.39-480; des weiteren Fehrenbach, Traditionale Gesellschaft, S.79f.; SchUbert, Fran- 
Besitz- und Herrschaftsrechten, d.h. die Verquickung der öffentlich-rechtlichen und privatrechtlichen Sphäre, die ein wesentliches Merkmal der Agrarverfassung gerade in den westfälischen Landesteilen des Großherzogtums war, ließ sich mit den eigentums- und personenrechtlichen Bestimmungen des französischen Zivilrechts nicht mehr in Einklang bringen. Durch die in der Feudalverfassung wurzelnden persönlichen Abhängigkeiten wurden die rechtliche Gleichheit und Freiheit der Person konterkariert. Sukzessionsregelungen standen der freien Verfügung über das Eigentum im Weg. Die Feudalstrukturen schränkten zudem den Herrschaftanspruch des Landesherrn auf seine Untertanen ein und behinderten die gleichmäßige Heranziehung aller Untertanen zu den öffentlichen Pflichten.

Für nicht kompatibel mit den Grundlagen, auf denen das französische $\mathrm{Zi}$ vilrecht beruhte, hielten die Reformbeamten zum einen die Lehnsordnung.

Schon der veränderte Geist der Zeit, hieß es in einem Gutachten des Staatsrats, und die jetzt ganz anders geformte Staatsverbindung dulden dieses alte Gebäude der Vorzeit nicht mehr, und noch weniger verträgt es sich mit der bevorstehenden neuen Organisation der großherzoglichen Provinzen und dem Geiste desjenigen Gesetzbuches, was Euerer Kaiserl. Königl. Majestät erhabenen Nahmen führt ${ }^{313}$.

Zum anderen galt das in den westfälischen Landesteilen immer noch weit verbreitete Institut der Leibeigenschaft ${ }^{314}$ als unvereinbar mit den geplanten Reformen, weil die Eigenhörigen nicht nur mit umfangreichen Diensten und Abgaben, wie dem Gesindezwang, dem Sterbefalle bzw. der Erbfolge, den Hand- und Spanndiensten sowie jährlichen Prestationen belastet, sondern darüber hinaus persönlich unfrei und rechtlich unmündig waren. Die Leibeigenschaft nähere sich einer modifizierten Sklaverei sehr, kritisierte Provinzialrat Schmitz in einer Denkschrift aus dem Jahre 1808, und steche gegen den in neuern Zeiten so allgemein gepriesenen Grundsatz: daß alle Unterthanen vor dem Gesetze gleich stehen, und die Menschen-Rechte unangetastet bleiben sollen, sehr grell $a b^{315}$.

Der Abbau der feudalrechtlichen Bindungen erwies sich allerdings als eine schwierige und äußerst prekäre Angelegenheit. Komplizierte und umstrittene Rechtslagen, schwer zu entflechtende Abhängigkeits- und Dienstverhältnisse

zösisches Recht, passim; zur Rolle des Staatsrats in dieser Frage siehe FrancKSEN, Staatsrat, S.61f.

313 Undatierter Rapport der Staatsratskommission über Inhalt und Ausdehnung des Lehnswesens, abgedruckt in: RoB, Regierungsakten des Großherzogtums Berg, S. 148-150, Zitat S.148.

314 Vgl. die Denkschrift des Steinfurter Provinzialrats Schmitz über die Leibeigenschaft vom 8. Mai 1808, abgedruckt ibid. S.39-51. In dieser Abhandlung schrieb Schmitz, daß in seinem Verwaltungsbezirk außer den Bewohnern der Städte und Flecken, den Köttern und einigen Hofbesitzern, welche sich losgekauft hatten, alle Einwohner entweder Leibeigene oder umgekehrt deren Grundherren seien, ibid. S. 40.

315 Ibid. S. 45. 
sowie divergierende Interessen von Grundherren und Bauern machten den Beamten die Arbeit schwer. Zudem berührten die geplanten Veränderungen vitale Interessen des Souveräns. Die Aufhebung von Leibeigenschaft und Lehnswesen seien deux articles également essentiels et fort délicats par leur voisinage du droit de propriété, et parcequ'ils intéressent ici à un haut dégré les Domaines et les droits du Souverain, erklärte Beugnot gegenüber Napoleon am 7. September $1808^{316}$. Der kaiserliche Kommissar warnte deshalb davor, dem französischen Beispiel uneingeschränkt zu folgen.

Le Grand-Duché obtiendra tous les bienfaits de cette révolution, meinte er statt dessen, sans les payer par des excés; et il faut pour cela respecter la propriété dans quelque mains qu'elle soit [...]. Il n'y auroit rien de gagné, si, en déplaçant seulement les forces, on changerait les opprimés en oppresseurs, et si on substituait une turbulence démocratique, au long sommeil de la servitude ${ }^{317}$.

Ende 1808 wurde das Projekt, an dem die Grundherren, aber nicht die Bauern mitgewirkt hatten, schließlich auf den Weg gebracht. Den Anfang machte die Abschaffung der Leibeigenschaft am 12. Dezember 1808318. Ihr folgte die Beseitigung der Lehnsherrschaft am 11. Januar 1809319.

Ein Kernbereich der neuen Gesetzgebung war die Liberalisierung des Eigentums entsprechend den inhaltlichen Verfügungen des französischen Gesetzbuches. Die Teilung von Grund und Boden in dominium directum und dominium utile wurde aufgehoben ${ }^{320}$ - ein für Deutschland einmaliger Akt. Zwischen Besitz- und Nutzungsrechten bestanden im Großherzogtum seither keine Unterschiede mehr. Darüber hinaus erklärte die Regierung alle im Großherzogtum bestehenden Lehen für abgeschafft, mochten sie unmittelbar von der Krone, oder von Privatlehnsherren abhängen ${ }^{321}$. Der Besitz ging in das freie Eigentum der Vassallen über, die es in Zukunft nach ihrem Gutbefinden benutzen, und darüber verfügen konnten ${ }^{322}$. Die lehnsrechtlichen Beschränkungen in der Erbfolge, allen voran die Substitution und das Fideicommiß, wurden ebenfalls aufgehoben. Die Erbfolge war künftig der durch die Gesetze des Staates eingeführten allgemeinen Erbfolgeordnung unterwor$\mathrm{fen}^{323}$.

316 Antwortschreiben Beugnots an den Pariser Finanzminister Gaudin vom 7.September 1808, AN, AF IV 1842.

317 Circular Beugnots an die Präfekten des Großherzogthums betr. die Anwendung des Leibeigenschafts- und Lehnsdekrets vom 26. Juli 1810, abgedruckt in: Roв, Regierungsakten des Großherzogtums Berg, S. 109-112, Zitat S. 112.

318 Gesetz-Bulletin, 1. Abtheilung, Nr. 6, abgedruckt in: ibid, S. 92-103.

319 Gesetz-Bulletin, 1. Abtheilung, Nr. 8, abgedruckt in: ibid, S. 161-193.

320 Gesetz-Bulletin, 1. Abtheilung, Nr. 6, Art. 2.

321 Ibid. Nr.8, Art. 1 .

322 Ibid. Art. 2.

323 Ibid. Art. 3. 
Zweiter Kernbereich der neuen Gesetzgebung waren Bestimmungen zur Gleichstellung und Liberalisierung der Untertanen. Hierzu gehörte einmal die Auflösung der persönlichen Bindungen, die dem Kolonat und dem Lehnsverhältnis entsprangen. Die ehemals Leibeigenen und Colonen sollen alle bürgerliche Rechte in ihrer ganzen Ausdehnung genießen, so wie alle andere Bewohner des genannten Großherzogthums, hieß es im Dekret zur Aufhebung der Leibeigenschaft ${ }^{324}$. Ebenso fielen der bisher von den Lehnspflichtigen zu leistende Eid und das daraus hervorgehende Treueverhältnis fort. Des weiteren wurden die auf der Person liegenden Pflichten entschädigungslos aufgehoben $^{325}$, weil nach dem Code Napoléon Servitute weder einer Person, noch zum Vortheil einer Person [...] auferlegt werden durften ${ }^{326}$. Zu den mit der Person verbundenen Pflichten zählte der Gesetzgeber vor allem den Gesindezwang, das Recht auf Freikauf, den Sterbfall, der nicht auf dem Kolonat haftete, alle Fronden, Hand- und Spanndienste, ferner alle auf der Person lastenden Lehnsdienstbarkeiten. Alle Rechte, die auf Grund und Boden selbst ruhten, wie der Sterbfall, die Erbgewinn-, Auffahrts- oder Weinkaufgelder, der Heimfall sowie alle Lehnsdienstbarkeiten, die weder der Person noch zu Gunsten der Person auferlegt waren ${ }^{327}$, galten demgegenüber als Reallasten und waren im Unterschied zum französischen Vorbild grundsätzlich ablösbar. Der Modus der Ablösbarkeit blieb dabei äußerst vage.

Neben der Demontage der ländlichen Sozialverfassung stellte die Abschaffung der korporativen Berufsordnung eine weitere wesentliche Vorbedingung für die Übernahme des französischen Zivilgesetzbuches dar, denn der Code Napoléon kannte nur noch besondere Gesellschaften, deren $\mathrm{Zu}$ sammenschluß sich auf den Nutzen oder Gebrauch bestimmter Sachen gründete. Hierunter fiel auch der Vertrag, wodurch mehrere Personen zu einer bestimmten Unternehmung, oder zur Ausübung eines Handwerkes, sich vereinigten $^{328}$. Am 31. März 1809 zog die Regierung daraus die Konsequenz und schaffte die berufsständischen Korporationen ab. Zwar sprach sie in ihrem Dekret zur Einführung der Patentsteuer die Auflösung der Zünfte nicht explizit an. Doch konnte jeder, der die Patentgebühr innerhalb der vorgeschriebenen Fristen bezahlte, im Großherzogtum künftig Handel oder Gewerbe treiben ${ }^{329}$. Dadurch ging den Zünften jegliche Existenzberechtigung verloren. Mit der Auflösung der korporativen Bindungen übernahm der Staat zugleich die Kontrolle über die Handwerker. Gesellen und Knechte waren seither zur Führung eines Büchelchens verpflichtet, welches enthält

324 Ibid. Nr. 6, Art. 1.

325 Ibid. Art. 3; ibid. Nr. 8, Art. 4.

326 Code Napoléon. Édition seule officielle pour le Grand-Duché de Berg, Düsseldorf 1810, Art. 686. Das französische Zivilrecht erlaubte nur, seine Dienste auf eine bestimmte Zeit oder für eine bestimmte Angelegenheit zu verdingen (Art. 1780).

327 Gesetz-Bulletin, 1. Abtheilung, Nr.6, Art.3f.; ibid. Nr. 8, Art.4f., Zitat: Art. 5.

328 Code Napoléon, Art. 1841f.

329 Gesetz-Bulletin, 1. Abtheilung, Nr. 15. 
seine Namen und Vornamen, sein Alter, seinen Geburtsort, seine Bezeichnung, seine Profession und den Namen des Meisters, bey welchem er [...] in Arbeit war ${ }^{330}$.

Die Einführung der Patentsteuer diente allerdings nicht nur dazu, die Zunftverfassung außer Kraft zu setzen. Sie war vielmehr zugleich Bestandteil einer umfassend angelegten Steuerreform. Diese richtete sich laut Beugnot auf den Abbau von Steuerbefreiungen und die Schaffung von mehr Steuergerechtigkeit. In Wirklichkeit aber ging es darum, das Steuervolumen zu verbessern. Die Notwendigkeit dazu ergab sich aus der Tatsache, daß die Besteuerung das wichtigste Instrument zur Haushaltsfinanzierung war, die durch die herkömmlichen Steuern erzielten Erträge aber schon seit langem und bei weitem nicht mehr ausreichten, um den Finanzbedarf des Staates und das unter Murat beträchtlich vergrößerte Defizit zu decken. Noch weniger genügten die fiskalischen Einnahmen dazu, die ambitionierten Reformabsichten Napoleons zu finanzieren. Die grundlegende Neugestaltung der Steuerverfassung war daher unvermeidlich, insbesondere wenn das Gelingen der französischen Herrschaftsziele nicht in Gefahr gebracht werden sollte.

Beugnot führte die unzureichenden Steuererträge vor allem auf Mängel in der Direktbesteuerung zurück. Alles sei in Unordnung, beanstandete er am 7.September 1808 bezüglich der Grundsteuererhebung, tout est à réparer [...]. La répartition est mauvaise, [...] des plaintes s'elevent de beaucoup d'endroits, tandis que le contingent est faible en soi ${ }^{331}$.

On ne s'est jamais occupé de rechercher, kritisierte er am 16.Januar 1809 die Industriesteuer, si l'impôt affectait les moyens de travail, compromettait la reproduction, ou en arrêtait le développement. [...] Il manque au Grand Duché, schloß er daraus, la première source de toute prosperité, un bon systême [sic] de revenu public ${ }^{332}$.

Um die Erträge aus der Direktbesteuerung zu verbessern und das Mißverhältnis zwischen Staatsausgaben und Steueraufkommen im Bereich der Direktsteuer auszugleichen, wünschte der Finanzminister die von Murat nur halbherzig verfolgte Neuaufnahme von Grund und Boden ${ }^{333}$. Darüber hinaus

\section{Ibid. 2. Abtheilung, Nr. 7.}

331 Antwortschreiben Beugnots an den Pariser Finanzminister Gaudin vom 7. September 1808, AN, AF IV 1842.

332 Beugnot in seinem Rapport pour l'établissement du droit de Patente dans le Grand Duché an Napoleon vom 16. Januar 1809, AN, AF IV* 460.

333 Antwortschreiben Beugnots an den Pariser Finanzminister Gaudin vom 7. September 1808, AN, AF IV 1842. Die Bemühungen der Regierung Murats zur Neuaufnahme von Grund und Boden waren an der Heterogenität der einzelnen Landesteile gescheitert. Weil in denen das jetzige Großherzogthum Berg ausmachenden Theilen desselben, ein verschiedener Geld-Cours, eine sehr abweichende Art des Landmaßes, des Getreide- und Holzmaßes, und der sonstigen Naturalschätzungen üblich ist, hatte Murat in seiner Verfügung vom 16. April 1807 angeordnet, so bleibt es [...] dabey bewenden, daß die Angabe der Größe der Grundstücke in das Aufnehmungs-Protokoll, nach der in der Gemeinde für die Grundstücke gewöhnlichen Größenmaße geschehen. Vgl. Scortr, Nr.2953, Art.19. 
machte er sich dafür stark, das heterogene Abgabensystem des Großherzogtums durch die französische Steuertrias zu ersetzen ${ }^{334}$ und zu diesem Zweck ebenfalls die in Frankreich erhobene Patent- und Mobiliarsteuer in Berg einzuführen ${ }^{335}$. Mit Hilfe der französischen Steuerverfassung wollte er zum einen alle Untertanen gleichmäßig an den öffentlichen Lasten beteiligen und neue Finanzquellen erschließen. Zum anderen versprach er sich davon klare und einheitliche Grundlagen für die Direktbesteuerung ${ }^{336}$.

Beugnot konnte seine Reformabsichten schnell verwirklichen. Seit April 1809 mußten, wie gesagt, sogenannte Patentgebühren statt der bisherigen Gebühren und Abgaben für das Betreiben von Handel, Gewerbe, Künste und Handwerk entrichtet werden ${ }^{337}$. Sie setzten sich aus einem tariflich bestimmten, dem ausgeübten Beruf entsprechenden Anteil und aus einem Zehntel des Gebäudemietwertes zusammen. Öffentliche Beamte, Hebammen, Posthalter, Ackersleute, stille Handelsgesellschafter, Tagelöhner und sonstige Arbeiter, Fischer, Schornsteinfeger, Abtritt-Räumer, Woll- und Baumwollkämmer, Spinnerinnen und Verkäufer von Eßwaren blieben unbesteuert.

Darüber hinaus trat zum 1.Januar 1810 die französische Mobiliarsteuer an die Stelle der von Murat eingeführten Familiensteuer ${ }^{338}$. Auch ihr lagen zwei Einzelsteuern zugrunde. Sie bestand aus einer Abgabe der Person in Höhe eines Francs und aus einer Mietsteuer. Letztere richtete sich nach dem Hauszins jedes Steuerbaren. Steuerpflichtig waren grundsätzlich alle Einwohner, die das Alter von 21 Jahren erreicht hatten. Jüngere Untertanen konnten besteuert werden, falls sie über eigenes Einkommen verfügten. Für Magazine, Läden, Kaffee- und Billiardstuben, öffentliche Ballsäle, Werkstätten, Hammer-, Hütten- und andere Werke, Gasthäuser und Häuser öffentlicher Beamter mußte keine Mietsteuer gezahlt werden. Arme, Geistliche mit einer Pension unter 400 Franken, in Lohn stehendes Gesinde, Militärpersonen sowie

334 Planungen zur Übernahme der französischen Direktbesteuerung hatte bereits die Vorgängerregierung eingeleitet. Das geht aus dem undatierten Schreiben Beugnots an den ehemaligen Finanzminister Agar (1809) hervor, AN, 40 AP 15.

335 Beugnot in seinem Rapport pour l'établissement du droit de Patente dans le Grand Duché an Napoleon vom 16. Januar 1809, AN, AF IV* 460. J'ai pensé que c'était par la suppression de ces taxes qu'il fallait commencer, begründete Beugnot seine Reformvorschläge, et je me suis occupé d'en proposer le remplacement par un impôt général, uniforme, modéré, approprié à l'état actuel et assez prospère de l'industrie dans le Grand Duché. J'ai recherché si parmi les taxes anciennes, il ne s'en trouverait pas de qui on pourrait emprunter quelques formes utiles, liées à des circonstances locales, mais [...] ne je n'ai rien trouvé qui dût échapper à la suppression. Vgl. zudem das undatierte Schreiben Beugnots an den ehemaligen Finanzminister Agar (1809), AN, 40 AP 15.

336 Beugnot in seinem Rapport pour l'établissement du droit de Patente dans le Grand Duché an Napoleon vom 16. Januar 1809, AN, AF IV* 460. Um möglichen Widerständen einheimischer Beamter aus dem Weg zu gehen, hatte er den Staatsrat von vornherein von den Planungen ausgeklammert. FranckSEN, Staatsrat, S.197f.

337 Gesetz-Bulletin, 1. Abtheilung, Nr. 15.

338 Ibid. Nr. 20. 
Bürger, die noch kein ganzes Jahr im Ort wohnten, waren von der Mobiliarsteuer vollständig befreit ${ }^{339}$.

Abgesehen von der Einführung der allgemeinen Steuerpflicht in der Direktbesteuerung führte die Regierung eine Reihe neuer indirekter Auflagen für die Einwohner ein. Hierzu zählten insbesondere Zölle sowie Abgaben auf Kolonialwaren und auf Konsumgüter wie Getränke und Seife ${ }^{340}$. In den Jahren 1811 und 1812 trat im Großherzogtum außerdem, wie schon erwähnt, die französische Salz- und Tabakregie in Kraft. Während Tabak daraufhin nur noch aus dem Empire importiert und von einem Hauptdepot in Düsseldorf verkauft werden durfte, wurde Salz ausschließlich von den innerstaatlichen Salinen in Königsborn und Sassendorf geliefert. Die Berechnung des Salzverbrauchs erfolgte pro Kopf $\mathrm{f}^{341}$.

Mit Einführung des Code Napoléon zum 1.Januar 1810 trat der Reformprozeß in ein neues Stadium. Die eigentliche Rezeption des französischen Zivilrechts begann, und das, was bis dahin nur für Teile der Bevölkerung Gesetzeskraft erlangt hatte, wurde auf die gesamte Einwohnerschaft ausgedehnt. Seither konnte jeder frei über seinen Besitz verfügen. Alle Inländer waren im Genuß der bürgerlichen Rechte ${ }^{342}$. Allerdings blieb die Gleichheit, die das neue Zivilrecht gewährte, beschränkt. Das galt vor allem im Hinblick auf die Gleichstellung der Geschlechter. Im Rahmen der Zivilehe räumte der Gesetzgeber dem Ehemann weitreichende Befugnisse gegenüber seiner Gattin ein ${ }^{343}$. Ebensowenig war mit der bürgerlichen Gleichheit zugleich die politische Gleichheit verbunden. Die Ausübung der bürgerlichen Rechte, hieß es in Artikel 7 des Zivilgesetzbuches, ist von der Eigenschaft eines Staatsbürgers unabhängig, welche letztere man nur nach den Vorschriften der Staatsverfassungsgesetze erwirbt und erhält ${ }^{344}$. Zur Gruppe der Staatsbürger zählten nur diejenigen, die aus dem Großherzogtum stammten, das Alter von 21 Jahren erreicht hatten, im Bürgerregister der Gemeinde eingeschrieben waren und seit mindestens einem Jahr dort wohnten ${ }^{345}$.

Komplementär zu ihren Egalisierungs- und Liberalisierungsbestrebungen im Rahmen der Zivilrechtsreform schützte und vereinheitlichte die Regierung ebenfalls die Stellung der Einwohner als Justizobjekte.

Zum Schutz der individuellen Sicherheit ordnete Nesselrode am 7. April 1810 an, es solle jeder, welcher verhaftet werde, entweder gleich vor den Ju-

339 Die Konfektion der Steuerlisten wurde den Maires anvertraut, während die Regierung das jährliche Steuerquantum bestimmte und anschließend auf die einzelnen Verwaltungsbezirke umlegte.

340 Vgl. das Kaiserliche Dekret vom 26. Januar 1812, in: Gesetz-Bulletin, 2. Abtheilung, Nr. 90.

$341 \mathrm{Vgl}$. die Abschriften der beiden Dekrete in der Akte AN, 29 AP 55.

342 Code Napoléon, Art. 8.

343 So konnte die Ehefrau ohne die Zustimmung ihres Mannes nicht vor Gericht erscheinen, ibid. Art. 215.

344 Ibid. Art. 7.

345 Gesetz-Bulletin, 2. Abtheilung, Nr. 3, Art. 3. 
stiz- oder Polizei-Beamten des Ortes, wo die Verhaftung geschieht, gebracht oder [...] diesem Beamten von der geschehenen Verhaftung unverzüglich die Anzeige gemacht werden, damit derselbe in Ansehung des Verhafteten das Geeignete verfüge. Wer einen andern gefangen hält, ohne der Vorschrift dieses Artikels genüget zu haben, soll, als des Verbrechens willkührlicher Gefangenhaltung schuldig, peinlich verfolgt werden ${ }^{346}$.

Daneben wurden im Rahmen der Justizreformen von 1811 die im altständischen Gefüge wurzelnde Privilegierung einzelner Bevölkerungsgruppen vor Gericht sowie die patrimoniale Gerichtsbarkeit beseitigt. Neben die bürgerliche Gleichheit trat ergänzend die Gleichstellung der Einwohner vor Gericht. Alle Untertanen des Großherzogtums sollten unterschiedslos, verordnete der Landesherr in Artikel 2 der neuen Justizverfassung, in gleichen Fällen in der nämlichen Form und vor den nämlichen Richtern Recht nehmen ${ }^{347}$. Darüber hinaus waren seitdem alle Untertanen einem einheitlichen Strafkanon unterworfen ${ }^{348}$.

Durch die von französischer Seite vorgenommenen Neuerungen wurden die Wirtschafts- und Gesellschaftsstrukturen des Großherzogtums grundlegend umgestaltet. Indem der Gesetzgeber die Einwohner aus ihren feudalrechtlichen bzw. korporativen Bindungen löste, indem er sie als rechtsfähige Subjekte anerkannte und ihnen in diesem Zusammenhang nicht nur gleiche Pflichten auferlegte, sondern erstmals auch gleiche Rechte zubilligte, trieb er die Auflösung der ständisch-korporativen Wirtschafts- und Gesellschaftsordnung erheblich voran und schuf Grundlagen für ein verrechtlichtes, auf Freiheit und Gleichheit beruhendes Sozialgefüge.

Aufgrund der unterschiedlichen Ausgangsbedingungen in den einzelnen Landesteilen war der Stellenwert der neuen Gesetzgebung jedoch nicht überall gleich. Während der Reformproze $B$ in rückständigen Landesteilen wie den westfälischen durch die Veränderungen vielfach erst in Gang kam, stießen die Reformen in fortschrittlichen Gebieten, allen voran im wirtschaftlich weit entwickelten Herzogtum Berg, häufig auf Gegebenheiten, die durch sie eigentlich erst geschaffen werden sollten. Abgesehen davon blieb die Gesetzgebung insgesamt widersprüchlich und begrenzt. Durch Ausnahmeregelungen, wie etwa die Möglichkeit, dem Dienst an der Waffe durch die Stellung eines Ersatzmannes zu entgehen, wurden weiterhin einzelne Gesellschaftskreise favorisiert - in diesem Fall die Vermögenden - und der Gleichheitsgrundsatz aufgeweicht. Das galt im übrigen auch für die Kriterien, durch welche der Gesetzgeber den Zugang zu den Vertretungsorganen auf den verschiedenen Ebenen der Verwaltung reglementierte. Einzelne Rechtsbereiche wurden von

346 Ministerieller Erlaß vom 7. April 1810, HStAD, JB, Hofrat A, Nr.159g, Bd. 3.

347 Gesetz-Bulletin, 2. Abtheilung, Nr. 52, Art. 2.

348 Code Pénal, Précédé de la loi sur l'administration de la justice et l'organisation des tribunaux, Paris 21810; siehe auch André Damien, Code Pénal, in: TularD, Dictionnaire Napoleon, S. 433-435. 
der Regierung überhaupt nicht angetastet und boten Platz für politische Interventionen ${ }^{349}$. Einen kodifizierten Katalog über die zugestandenen Rechte gab es auch nicht.

\subsubsection{Verbindlichkeit der neuen Rechtsbestimmungen}

Es bleibt abschließend zu klären, welche Bedeutung und Funktion die Gesetzgebung im täglichen Leben erlangte. Ein für die Beantwortung dieser Frage zentraler Aspekt ist die Verbindlichkeit der neuen Rechtslage im Altag. Die Frage, ob, und falls ja, wie weit beziehungweise in welcher Form Einwohner, Behörden und Regierung die neu eingeführten Gesetze respektierten, gibt nämlich nicht nur Auskunft über deren Akzeptanz in der Bevölkerung und den Reformwillen der Beamtenschaft, sondern zugleich über den Grad an Rechtsstaatlichkeit der französischen Herrschaft insgesamt. Die Verbindlichkeit, die die neue Gesetzgebung tatsächlich besaß, soll deshalb Thema der folgenden Ausführungen sein.

Normabweichendes Verhalten stellte im Großherzogtum keine Seltenheit dar, sondern war im Gegenteil ein Phänomen, das den Rezeptionsprozeß der neuen Gesetzgebung bis 1813 begleitete. Nicht jede Nonkonformität war dabei zwangsläufig ein Gesetzesübertritt. Die Grenzen zwischen Legalität und Illegalität waren vielmehr fließend. Neben der eigentlichen Rechtsverletzung gab es eine ganze Bandbreite von Möglichkeiten, um sich Gesetzen zu entziehen.

\subsubsection{Normverstöße der Einwohner}

Zunächst waren es die Betroffenen selbst, die sich nicht immer an die gesetzlichen Spielregeln hielten, und ein Feld, in dem sie häufig auffällig wurden, war die Konskription. Wehrpflichtige legten gefälschte medizinische Atteste vor oder verstümmelten sich, um dem Dienst an der Waffe zu entgehen ${ }^{350}$. In der ehemaligen Grafschaft Mark beispielsweise schnitten sich junge Männer den Zeigefinger $a b$ und setzten damit eine Praxis fort, die noch aus preußischer Zeit stammte, wie Gaudin dem französischen Kaiser am 28. März 1809 berichtete $^{351}$. Andere wiederum entzogen sich der Konskriptionspflicht durch Nichterscheinen bei der Ziehung oder Fahnenflucht ${ }^{352}$.

Darüber hinaus versuchten Einwohner immer wieder, die Steuergesetze zu umgehen, und besonders häufig verstießen sie hierbei gegen die indirekte

349 So machte Beugnot kein Geheimnis daraus, daß er die Überwachung der Post für notwendig hielt. Sans doute, schrieb er Napoleon in seinem Brief vom 3. Januar 1809, il faut surveiller la correspondance, mais avec mesure, avec secret, avec précaution que le public ait sur ce point des soupçons vagues; on ne peut les lui ôter nulle part; mais l'individu ne doit pas avoir dans les mains la preuve physique de l'action de la police sur les Postes (AN, AF IV* 460).

350 Schreiben des Präfekten Romberg an die Unterpräfekten und Maires des Ruhrdepartements vom 16. Februar 1810, abgedruckt in: PVRD 1810, S.24.

351 Bericht Gaudins an Napoleon vom 28. März 1809, HStAD, Handschriften, B II, Nr. 5.

352 Ausführlich dazu: KandiL, Sozialer Protest, S.59f.; ferner Dreher, Truppen, S. 54. 
Besteuerung. Die von der Zollverwaltung errichteten Pfähle zur Anzeige der Zollstätten wurden niedergerissen und Douaniers tätlich angegriffen ${ }^{353}$. Der Schmuggel blühte. Darüber hinaus hatten die Staatsdiener mit Verstößen im Bereich der Direktbesteuerung zu kämpfen. Handel- und Gewerbetreibende lösten kein Patent für die Ausübung ihrer Tätigkeit, so daß die Patentsteuererträge die von Beugnot veranschlagte Summe von 550000 Francs bei weitem nicht erreichten ${ }^{354}$. Grundbesitzer machten im Rahmen der Aufnahme des Bodens falsche Angaben. Hierbei kam ihnen zugute, daß Grund und Boden nicht neu vermessen wurden, sondern ihre Informationen ausschlaggebend waren. In mehreren Grundstückserklärungen habe er mit großem Mißfallen bemerkt, kritisierte etwa Präfekt Romberg, daß verschiedene Pächter sich erlauben, die Größe der Grundstücke geringer, als sie wirklich ist, anzugeben ${ }^{355}$.

Die Umgehung der Konskriptions- und Steuergesetze war wohl nur gelegentlich auf Unkenntnis der neuen Rechtslage zurückzuführen ${ }^{356}$. In der Regel handelte es sich um bewußt begangene Aktionen, deren tiefere Ursachen in der zunehmenden Vereinnahmung der Untertanen durch den Staat zu suchen sind. Dafür spricht einmal, daß vergleichbare Probleme bereits aus vorfranzösischer Zeit überliefert sind. Dafür spricht ebenfalls, daß Versuche, sich den Auflagen des Staates zu entziehen, dort besonders ausgeprägt waren, wo es diese in vorfranzösischer Zeit nicht gegeben hatte. Dafür spricht schließlich auch, daß es sich nicht um ein bergisches Spezifikum handelte, sondern um ein typisches Problem des französischen Kaiserreichs. Beispielsweise war die Renitenz gegen die Konskription keine Besonderheit des Großherzogtums, sondern auch in anderen Teilen des Empire zu beobachten - in Frankreich selbst ebenso wie in anderen Staaten ${ }^{357}$. Es wäre deshalb auch verfehlt, die Verstöße als Angriff auf den Gleichheitsgrundsatz zu deuten oder darin eine grundsätzliche Oppositionshaltung gegen die französische Herrschaft zu sehen ${ }^{358}$.

353 Schreiben des Präfekten Borcke an die Unterpräfekten und Maires des Rheindepartements vom 18. Februar 1810, abgedruckt in: PARD 1810, S.68; Schreiben des Präfekten Romberg an die Unterpräfekten und Maires vom 20. Februar 1810, abgedruckt in: PVRD 1810, S. $26 f$.

354 Schmid, Grand-Duché, S. 289.

355 Bekanntmachung des Präfekten Romberg vom 22. Juni 1809, abgedruckt in: PVRD 1809, S.15.

356 Er habe mehreren Reklamationen entnommen, schrieb Präfekt Borcke den Maires seines Verwaltungsbezirks Anfang 1811, daß die Steuerpflichtigen weder mit der Art der Vertheilung der Personal- und Mobiliarsteuer noch mit der Anfertigung der Mutter- und Heberollen hinreichend vertraut seien und auch keinen voliständigen Begriff von dem Verfahren bey der Untervertheilung auf die Contribuablen hätten. Schreiben des Präfekten Borcke an die Unterpräfekten und Maires des Rheindepartements vom 20. Februar 1811, abgedruckt in: PARD 1811, S.38f., Zitat S. 38.

357. Vgl. ForRest, Déserteurs, S.57f.

358 Für KandIL, Sozialer Protest, S. 58, waren die Verstöße gegen die Konskriptionsgesetze ebenfalls politisch motiviert. 
Die Verstöße der Einwohner gegen die Auflagen des Staates führten zu beachtlichen Steuerausfällen und Schwierigkeiten bei der Aufstellung des Heeres. Zudem wirkten sie sich auf die öffentliche Sicherheit spürbar aus. Die Regierung unternahm deshalb auch zahlreiche Anstrengungen, um von der Bevölkerung die Einhaltung der Gesetze zu erzwingen, und machte selbst vor drakonischen Repressionsmaßnahmen nicht halt. Ihre Disziplinierungsversuche brachten jedoch nicht die gewünschten Erfolge. So ließen sich die Einwohner nicht davon abhalten, bei der Grundsteuererhebung falsche Angaben über ihren Besitzstand zu machen, um den Steuerfuß so gering wie möglich zu halten. Offensichtlich wurden die fehlerhaftesten Angaben hierbei in den Reihen des Kommunalpersonals selbst gemacht.

Il y a des exemples, schrieb Arnold Mallinckrodt im April 1813, que des Maires, des conseillers municipaux et d'autres employés sont justement ceux, qui ont le plus caché, et dont les déclarations ont été les plus inexactes quant à la mesure et la classification des terres; et qui en général ont donné lieu à une fausse évaluation des propriétés situées dans un district où ils ont des biens ${ }^{359}$.

Aufgrund der Mißstände bei der Steuererhebung konnte die Steuermatrikel insgesamt nicht vereinheitlicht werden. Eine aktualisierte Katasterordnung kam bis zum Ende der französischen Herrschaft auch nicht zustande. Dennoch machte die Erhebung der Grundsteuer Fortschritte. Auf Grundlage der Neuaufnahme des Bodens verbesserte sich der Verteilungsprozeß. Darüber hinaus wurde die Erhebung der Steuer rationalisiert. Stadt und Land trugen gleichermaßen zu den Lasten bei. Auch ließ das neue Erhebungsmuster keine Steuerbefreiungen mehr zu, selbst wenn ehemals Befreite wie Geistliche und Schullehrer sich immer wieder um die Reaktivierung ihrer auBer Kraft gesetzten Vorrechte bemühten ${ }^{360}$.

Die Erhebung der Patentsteuer blieb ebenfalls unbefriedigend ${ }^{361}$, obwohl Verstöße zügig geahndet wurden, sobald die Behörden davon erfuhren. Nachdem etwa Präfekt Spee davon unterrichtet worden war, daß der Friedrich Sommer von Pfahl und der Friedrich Sommer von Oberwiel im Jahre 1812 mit Schweinen gehandelt, ohne ein Patent gelöst und die tarifmäßige Gebühren abgetragen zu haben, ordnete er unverzüglich an, die beiden straffällig Gewordenen für das, als Schweinhändler getriebene Gewerb, als Strafe zu dem doppelten tarifmäßigen Betrage anzuschlagen ${ }^{362}$. Eine ähnliche Auflage erhielt ein Gewerbetreibender aus Aachen, der ohne Patent mit Waren hausiert hatte ${ }^{363}$.

359 Mallinckrodt in einer der Schriften, die er Roederer am 8. April 1813 in Paris überreichte, AN, AF IV 1837.

360 Schreiben des Präfekten des Ruhrdepartements an die Unterpräfekten und Maires vom 12. April 1810, abgedruckt in: PVRD 1810, S. 92.

361 Beschluß des Präfekten Borcke vom 1. März 1812, abgedruckt in: PARD 1812, S.128; Beschluß des Präfekten Spee vom 13. Juli 1812, abgedruckt ibid. S. 274.

362 Beschluß des Präfekten des Rheindepartements vom 28. September 1812, abgedruckt ibid. S.363f.

363 Ibid. 
Den Widerstand im Konskriptionswesen bekam die Regierung gar nicht in den Griff. Trotz der Repressalien ließen sich die Einwohner nicht davon abhalten, den Dienst an der Waffe zu verweigern. Durch die Kriegsfurcht der Bevölkerung, die durch den Spanienfeldzug und erst recht während des Rußlandfeldzugs erheblich geschürt wurde, stieg die Zahl der Deserteure und Refraktäre sogar sprunghaft an $^{364}$. Offenbar nahmen die bergischen Konskribierten eher harte Strafen in Kauf als das Risiko, auf dem Schlachtfeld zu sterben. Hinzu kam, daß sie auf den Schutz der übrigen Einwohner hoffen konnten. Fälle, in denen Konskribierte bei Gemeindebewohnern Unterschlupf fanden oder von diesen beschäftigt wurden, waren keine Seltenheit ${ }^{365}$. Nach den Unruhen zu Beginn des Jahres 1813 waren die Widerstände gegen die Konskription überhaupt nicht mehr zu bremsen, obwohl Refraktären und Deserteuren seit dem Frühjahr 1813 die Todesstrafe drohte. Am 25. März 1813 teilte General Lemarois Roederer mit, von 3000 Wehrfähigen seien lediglich 1328 , also weniger als die Hälfte, erschienen ${ }^{366}$.

Gegen den Schmuggel besaß die Regierung ebensowenig eine Handhabe. Begünstigt durch die Solidarität in der Bevölkerung und die nicht von der Hand zu weisende Komplizenschaft zwischen Einwohnern und Verwaltungsdienern sowie genährt durch die ständig wachsenden Ansprüche des Kaisers, stieg die Renitenz der Untertanen unaufhaltsam. Als fataler Fehler sollte sich in diesem Zusammenhang der Schritt erweisen, im Großherzogtum Berg auch noch die in Frankreich übliche Salz- und Tabakregie einzuführen. Bereits kurz nach Erlaß der neuen Auflagen setzte ein reger illegaler Handel mit Salz und Tabak ein. Banden schlossen sich zusammen, um mit den verbotenen Waren zu handeln. Viele Einwohner begannen ohnehin, ihren Konsum einzuschränken oder Kräuter aus eigenen Pflanzungen zu rauchen ${ }^{367}$. Die Ergebnisse der Tabak- und Salzregie blieben somit weit hinter den Erwartungen der Düsseldorfer Regierung zurück.

Enfin, le sel, le tabac [...] me mettent aux abois, klagte Beugnot gegenüber Roederer am 24. August 1812. Quelque soit le but politique qu'on se soit proposé en établissant le monopole ici, fuhr er an anderer Stelle fort, que ce but ait été simple, comme de fournir une branche de revenu de plus au Grand-Duché, ou que ce but ait été composé, comme de fournir le revenu et encore de défendre la frontière de France; il est évident que ni l'un ni l'autre ne seront bientôt atteints ${ }^{368}$.

364 Vgl. Dreher, Truppen, S.54f.

365 Vgl. die Schreiben Nesselrodes an das Düsseldorfer Tribunal der ersten Instanz vom 11. November 1811 und 16. Juli 1812, StAM, Großherzogtum Berg, A3, Nr. 4. Immer wieder wurden auch Fälle bekannt, in denen französische Refraktäre im Großherzogtum versteckt wurden. Vgl. die Verfügung Beugnots vom 1. Oktober 1808, Depositum Stadt Ratingen 1, ferner das Urteil des Düsseldorfer Hofrats vom 5. Juni 1811, HStAD, JB, Hofrat B II, Nr. 124, sowie das Urteil des Düsseldorfer Hofrats vom 13. August 1811, HStAD, JB, Ober-Appellations-Gericht BXIV 23.

366 Schreiben des Generals Lemarois an Roederer vom 25. März 1813, AN, AF IV 1226.

367 Bericht des Zollverwalters David vom 23. März 1812, AN, AF IV 1853B.

368 Brief Beugnots an Roederer vom 24. August 1812, AN, 29 AP 39. 
Die Entwicklungen im Schleichhandel führten nicht nur zu Steuerausfällen, sondern wirkten sich auch nachhaltig auf die öffentliche Sicherheit aus. Da die Zolldiener den Schmugglern vielfach zahlenmäßig unterlegen waren und zunehmend Gebrauch von ihrer Waffe machten, häuften sich die gewalttätigen Auseinandersetzungen zwischen Kontrebandiers und der Staatsgewalt. Ein Blick in die Akten genügt, um sich davon zu überzeugen ${ }^{369}$. Nicht selten endeten diese Konflikte tödlich, denn auch die Gewaltbereitschaft der Schmuggler stieg.

Nos Douanes et les fraudeurs, kommentierte Beugnot die Zuspitzung im Zollwesen, font une petite guerre, qui a pour résultat quelques hommes tués, beaucoup d'autres blessés, et toute une population démoralisée et préparée pour l'avenir à tous les genres d'excès: le tout sans qu'aucun profit pour les finances de l'Etat console, si on se pouvait consoler de cet effayant désordre ${ }^{370}$.

Der kaiserliche Kommissar sah sich durch das Fiasko in der indirekten Besteuerung im nachhinein darin bestätigt, daß die Tabakregie für das Großherzogtum Berg ungeeignet war.

La lecture de cette triste gazette, teilte er Roederer im Juli $1812 \mathrm{mit}$, confirme pour la 100m. fois, en attendant que ce soit pour la 1,000. ce que le Commissaire avait dit, et ce qu'il a même osé répéter devant S.M. l'Empereur, que le monopole du tabac était impracticable dans un pays qu'il était physiquement impossible de défendre contre les importations étrangères ${ }^{371}$.

Mehrere Indizien sprachen nach Beugnot dafür, daß die Eskalationen im Bereich der indirekten Steuererhebung auf den Schulterschluß zwischen Einwohnern und lokalen Verwaltungsdienern zurückzuführen waren. Zum ersten wurden ihm zufolge nicht die Zolldiener ungenügend bestraft, sondern die Schmuggler zu milde behandelt ${ }^{372}$. Zum zweiten arbeiteten die Munizipalbehörden nicht genügend mit Zolldienern und Gendarmen zusammen, und zum dritten spielten die Munizipalleiter in ihren Berichten das Problem herunter. Sie behaupteten, es gebe in ihren Mairien keine Anzeichen von Schmuggel, obwohl zahlreiche Belege das Gegenteil bewiesen ${ }^{373}$.

Um die Entwicklungen im Zollwesen wieder unter Kontrolle zu bringen, verschärfte Beugnot im Sommer 1812 die Überwachung der Unterbehörden. Er verpflichtete die Präfekten dazu, ihm wöchentlich über die Entwicklungen und Vorfälle im Schleichhandel Bericht zu erstatten. Falls sie diese Aufgabe nicht erledigten, drohte der kaiserliche Kommissar damit, den Kaiser einzu-

369 Vgl. u. a. die Berichte des Zollverwalters David an Roederer vom 31.Januar 1812, vom 18. Oktober 1812 und vom 8. November 1812, AN, AF IV 1853 ${ }^{\mathrm{B}}$, das Schreiben Beugnots an Roederer vom 11.Juni 1812, AN, 29 AP 58, sowie die Monatsberichte Beugnots an Roederer für Juni, Juli und August 1812, AN, 29 AP 38.

370 Monatsbericht Beugnots an Roederer für Juli 1812, ibid.

371 Ibid.

372 Schreiben Beugnots an den Generalprokurator Sethe vom 7. September 1812, ibid.

373 Schreiben Beugnots an den Präfekten Spee vom 28. September 1812, HStAD, GB, Nr.11027. 
schalten $^{374}$. Darüber hinaus veranlaßte Beugnot die Kollektivbestrafung der Kommunen für die auf ihrem Gebiet begangenen Defraudationen ${ }^{375}$ und kündigte drakonische, die Rechtssicherheit der Untertanen aufs Spiel setzende Repressionsmaßnahmen an. Sollte es zu Volksaufläufen kommen, wollte er Soldaten einrücken lassen, welche auf Kosten der Einwohner so lange allda verbleiben sollen bis die Urheber, Räthelsführer und alle, welche thätigen Antheil daran nahmen, oeffentlich bestraft sind ${ }^{376}$. Gegen Schleichhändler und ihre Komplizen sollten außergerichtliche Verfahren angestrengt werden. In all diesen Eingriffen sah Beugnot indes nur Palliativmaßnahmen. Endgültig gelöst wurde das Problem für ihn nur dann, wenn auch die Anrainerstaaten das Steuersystem des Großherzogtums einführten ${ }^{377}$.

Ebenso wie der kaiserliche Kommissar waren die Untertanen davon überzeugt, daß die Gerichte ihre Aufgaben nicht hinlänglich erfüllten. Allerdings meinten sie im Gegensatz zu Beugnot, daß nicht die Einwohner, sondern die Zolldiener unzureichend bestraft wurden. Deshalb fühlten sie sich auch mehr und mehr dazu autorisiert, sich selbst zu ihrem Recht zu verhelfen. Man glaube nicht, beschrieb Präfekt Spee Anfang Oktober 1812 diese Entwicklungen, que les douaniers qui outrepassent ou qui font des exces soient puni ce qui a le plus funeste effet sur l'esprit public et qui doit faire naitre l'idée de se faire droit soi meme [sic] puisque le gouvernement n'en donne pas ${ }^{378}$.

Der Schritt der Einwohner, sich selbst Recht zu verschaffen, markierte einen qualitativen Wandel im Handeln und Bewußtsein der Öffentlichkeit. Auf der einen Seite brachten die Untertanen damit offen ihre Enttäuschung und ihr schwindendes Vertrauen in den Staat und seine Institutionen zum Ausdruck. Auf der anderen Seite griffen sie aktiv ins politische Geschehen ein, um den Lauf der Dinge zu ihren Gunsten zu beeinflussen und zu steuern. Darüber hinaus stellten sie erstmals grundsätzlich die Rechtmäßigkeit der landesherrlichen Gesetzgebung und damit die Zuständigkeit des Staates überhaupt in Frage, denn sie schufen einen Raum, in dem nicht die von der Regierung kodifizierten und oktroyierten Gesetze galten, sondern ihre eigene Auffassung von dem, was recht und billig war. Aus dieser Sicht verloren ihre Aktionen auch ihren rechtsverletzenden Charakter. Sie waren legitimer Widerstand gegen die Staatsgewalt.

\subsubsection{Rechtsverletzungen durch öffentliche Amtsträger}

Ebenso wie die Betroffenen verstießen öffentliche Amtsträger gegen die neue Gesetzgebung. Ein von ihnen immer wieder begangenes Delikt war die Verletzung von Eigentumsrechten bei Hausdurchsuchungen. Visitationen, die

374 Schreiben Beugnots an den Präfekten Spee vom 31. Juli 1813, ibid.

375 Ibid.

376 Beugnot in seinem Schreiben an den Präfekten Spee vom 11. Juni 1812, ibid.

377 Monatsbericht Beugnots an Roederer für Juni 1812, AN, 29 AP 39.

378 So der Präfekt Spee in seinem Schreiben an Beugnot vom 1. Oktober 1812, HStAD, GB, Nr.11027. 
grundsätzlich ein legitimes Mittel zur Sicherung von Ruhe und Ordnung waren, erfolgten ohne die gesetzlich vorgeschriebene Hinzuziehung der Maires oder Beigeordneten. In verschiedenen Mairien beispielsweise machten Zollbeamte Hausdurchsuchungen nicht, wie vorgeschrieben, mit dem Maire oder den Beigeordneten, sondern zwangen zwei Mitglieder des Munizipalrats dazu, an den Visitationen teilzunehmen. Hausdurchsuchungen wurden außerdem willkürlich durchgeführt. Häufig reichte der geringste Verdacht, um ein Haus durchsuchen zu lassen, und selbst die Munizipaldiener waren davor nicht geschützt. Zum Beispiel wurde das Haus des Maires von Datteln und Waltrop, Boehne, auf die Denunziation hin inspiziert, Boehne lagere gesetzwidrig Tabak und Salz ${ }^{379}$. Immer wieder kam es zu nächtlichen Visitationen, obwohl eine solche Intervention nur tagsüber erlaubt war ${ }^{380}$. Der Polizeikommissar von Mülheim an der Ruhr, Guilleaume, beispielsweise nahm zusammen mit der Gendarmerie Hausdurchsuchungen wiederholt nachts vor, um versteckt gehaltene Konskribierte aufzuspüren ${ }^{381}$. In Düsseldorf erschienen mehrere Zollbeamte im Oktober 1810 morgens um ein Uhr bei dem Fischer David Wedel, um dessen Haus nach Kaffee und Zucker zu durchsuchen. Ihr Vorgehen rechtfertigten sie mit dem Argument, Wedels Name sei dreimal auf der Verdächtigenliste des Präfekten aufgeführt gewesen ${ }^{382}$. Bei Visitationen wurden zudem Waren unrechtmäßig konfisziert ${ }^{383}$.

Neben den Übergriffen auf Eigentumsrechte verstießen Staatsdiener wiederholt gegen persönliche Freiheitsrechte bzw. das Recht der Einwohner auf Unverletzlichkeit. Willkürliche und auch zu lange Inhaftierungen gehörten zur Tagesordnung. So hielt der Prokurator Sybel Anna Catharina Henrichs, die zusammen mit drei weiteren Personen wegen der Herstellung falscher Münzen verhaftet worden war, vom 15.Januar bis zum 29. April 1813 gefangen, obwohl ein Freilassungsbescheid des zuständigen Gerichts der 1. Instanz für den 14.Januar 1813 vorlag $^{384}$. Der Ausbruch von Unruhen im Februar 1813 löste geradezu eine Verhaftungswelle aus. Mehr als 120 Personen wurden - häufig ohne Verhör - von der Gendarmerie willkürlich gefangengenommen ${ }^{385}$.

379 Schreiben des Präfekten Spee an Beugnot vom 1. Oktober 1812, ibid.

380 Da das Haus eines jeden Bürgers während der Nacht eine unverletzliche Freystätte ist, hieß es etwa in den Vorschriften der Gendarmerieverordnung vom 22. November 1808, so soll die Gendarmerie nur im Falle einer Feuersbrunst, einer Ueberschwemmung; oder einer aus dem Innern des Hauses an die Gendarmerie gelangenden Aufforderung, sich hinein begeben dürfen. Vgl. HStAD, Depositum Stadt Ratingen 56.

381 Siehe den Beschluß Nesselrodes vom 11. April 1808, HStAD, GB, Nr. 4481.

382 Schreiben des Düsseldorfer Beigeordneten Heinrich Schnabel an den Präfekten Borcke vom 16. Oktober 1810, Vgl. HStAD, GB, Nr.11027.

383 Reisebericht des Präfekten Mylius vom 11. August 1810, HStAD, GB, Nr. 4400.

384 Antrag des Advokaten Wardenburg vom 17. Oktober 1813, HStAD, GB, Nr. 6392.

385 Bericht Sethes an Roederer vom 10. April 1813, AN, AF IV 1840. Zu willkürlichen Verhaftungen soll es vor allem in Elberfeld und Bensberg unter der Leitung des Kapitäns Krane am 30. Januar und 3.Februar 1813 gekommen sein. Vgl. Roederers Projet de rapport sur les operations des autorités judiciaires à l'occasion des troubles an Napoleon vom Februar 1813, AN, 29 AP 37. 
Darüber hinaus erfolgten Bestrafungen unrechtmäßig oder ohne Mitwirkung der Gerichte. Außerdem waren Körperverletzungen keine Seltenheit in den Quellen ist entweder von Züchtigung oder von körperlicher Mißhandlung die Rede. Ohne die Justizbehörden einzuschalten, arretierte und züchtigte der provisorische Polizeikommissar Soests, Martin, am 19. Juni 1811 zwei Männer unter dem Verdacht, einem Gefangenen des Soester Gefängnisses zur Flucht verholfen haben zu wollen ${ }^{386}$. Der Barmer Polizeikommissar De Witt setzte Anfang Januar 1811 dem Seidenweber und Tagelöhner Hermann Arm- und Daumenschrauben auf. Er bezichtigte Hermann mehrfachen Diebstahls ${ }^{387}$. Nicht zimperlich war man auch in Ewersbach im Siegdepartement. Man habe verdächtige Personen, teilte der Maire dem Präfekten mit, gleich über die Grenzen gebracht und ihnen zuweilen noch einige Schläge mit auf die Reise geben lassen ${ }^{388}$.

Die Douaniers waren für ihre Neigung zur Körperverletzung besonders bekannt, weshalb sie im Siegdepartement als Buveurs de sang geschmäht wurden ${ }^{389}$. Der Zolleinnehmer Thiebes schlug einem Mann mit der Faust, sey es auf den Munde, oder die Nase, nachdem er ihn zunächst verbal beleidigt hatte. Anschließend verhaftete er ihn auf eigene Faust ${ }^{390}$. In Kaiserswerth versetzte ein Douanier am 9. November 1812 einem Jungen einen Streich, weil sich dieser weigerte, einen Passierschein vorzuzeigen ${ }^{391}$.

Etliche Fälle von Körperverletzung sind auch für Gendarmen überliefert, obwohl es diesen nach $\S 42$ der Gendarmerie-Verordnung strikt untersagt war, bei Verhaftungen, Einsperrungen oder Exekutionen Härte anzuwenden ${ }^{392}$. Zum Beispiel schlug in Düsseldorf ein Gendarm einem Mädchen mit einem Stock über den Kopf und Gesicht dergestalten [...], daß das Blut darnach floß, wie der Düsseldorfer Polizeikommissar dem Kolonel der Gendarmerie am 11. April 1808 berichtete ${ }^{393}$.

Die in den Reihen der Amtsträgerschaft begangenen Eingriffe in geschützte Rechtsbereiche, wie nächtliche Hausdurchsuchungen oder Körpermißhandlungen gingen teils auf Unwissenheit oder alte Praktiken und Gewohnheiten zurück und machten deutlich, wie tief überlieferte Werte- und Handlungsmuster im Bewußtsein der Einwohner vielfach noch verankert waren.

386 Protokoll der Staatsratssitzung vom 7. Mai 1812, HStAD, GB, Nr. 319.

387 Protokoll der Vernehmung des Polizeikommissars durch den Richter Schramm vom 29. Mai 1811, HStAD, GB, Nr. 10899.

388 Tagebuch der Rundreise des Präfekten des Siegdepartements für 1810, HStAD, GB, Nr. 4410.

389 Brief Beugnots an den Generalprokurator Sethe vom 7. September 1812, AN, 29 AP 38.

390 Urteil des Hofrats vom 21. Juni 1811, HStAD, JB, Hofrat A, Nr. 91.

391 Bericht des Kaiserswerther Maires Herschbach an den Präfekten Spee vom 14.September 1812, HStAD, GB, Nr. 11027.

392 Ministerielle Verordnung wegen der für das Großherzogthum Berg errichteten Gendarmerie vom 22. November 1808, HStAD, Depositum Stadt Ratingen 56.

393 Bericht des Düsseldorfer Polizeikommissars an den Kolonel der Gendarmerie vom 11. April 1808, HStAD, GB, Nr. 6197. 
Teils lagen sie am schlechten Ausbildungsstand der Funktionsträger. Dies hing wiederum damit zusammen, daß bestimmte Amtsfunktionen in der Öffentlichkeit nur geringes Sozialprestige besaßen und deshalb auch nur wenig begehrt waren. Aus diesem Grund mußten die Staatsbehörden häufig mit Personen Vorlieb nehmen, die nicht über die nötigen Voraussetzungen und Qualifikationen verfügten. Das galt für Zolldiener ${ }^{394}$ und noch mehr für Gendarmen ${ }^{395}$.

Verschiedene Übertretungen waren zudem systemimmanent. Zum Beispiel hingen die Verstöße gegen Eigentumsrechte, die von Zolldienern begangen wurden, auch damit zusammen, daß diese bei der Beschlagnahmung von Schmuggelwaren einen Anteil des Warenwerts erhielten ${ }^{396}$. Daher hatten sie selbst großes Interesse an der Konfiskation von Waren. Rechtsverstöße in den Reihen der Gendarmerie wurden dadurch begünstigt, daß Gendarmen keine örtlichen Vorgesetzten besaßen, sondern unter militärischer Kuratel standen. Sie waren daher nur schwer kontrollierbar. Der Leiter des Öffentlichen Ministeriums war darüber hinaus der Ansicht, daß sprachliche Mängel in der Übersetzung der Gendarmerie-Ordnung für die gesetzlichen Überrtretungen von Gendarmen mitverantwortlich waren ${ }^{397}$. Ein Grund für die Korruptionsanfälligkeit der lokalen Bediensteten war dagegen, darauf ist bereits an anderer Stelle hingewiesen worden, die Ehrenamtlichkeit bzw. schlechte Bezahlung in der Munizipalverwaltung.

In der Regel wurden die Rechtsverletzungen durch die Betroffenen selbst bekannt - ein weiteres Zeichen dafür, daß die Einwohner Kenntnis und Bewußtsein von ihren Rechten hatten und auch Anspruch darauf erhoben. Der Seidenweber Hermann etwa widerrief vor Gericht Teile seines im Verhör gemachten Geständnisses mit dem Hinweis, Polizeikommissar De Witt habe ihn gefoltert.

Ich gestehe aufrichtig mein Vergehen, erklärte er, aber alles das, was ich vor dem Herrn Polizeiy-Commissair ausgesagt, ist nicht wahr, dieser hat mich auf eine fürchterliche und unerhörte Art zum Geständniß gezwungen, er setzte mir nämlich Daum- und Armschrauben auf, schraubte 3 ad 4 mal herum, bis ich schrie, ich wolle gestehen [...]. Dieses Schrauben geschah 2 Tage ${ }^{398}$.

394 Schreiben des Zollverwalters David an Roederer vom 16. Oktober 1812, AN, AF IV $1853^{B}$

395 Vgl. u. a. folgende Akte: Diejenigen Gendarmen, welche wegen unanständigen Betragens oder schlechten Lebenswandels entweder gänzlich entlassen oder zur Infanterie oder Cavalerie versetzt sind, HStAD, GB, Nr.6197. Die schlechte personelle Zusammensetzung des Gendarmeriekorps war der Regierung seit langem bekannt. Schon Anfang April 1807 hatte Nesselrode den Gendarmen alles Saufen, Schwelgen, und Spielen untersagt und ihnen verboten, die Untertanen mit Schlägen, noch sonst auf irgend eine Art zu misshandeln. Undatiertes Schreiben Nesselrodes an den Düsseldorfer Provinzialrat (April 1807), HStAD, GB, Nr.12685.

396 Gesetz-Bulletin, 2. Abtheilung, Nr. 89, Art. 43.

397 Schreiben Sethes an den Gendarmerie-Oberst Goltstein vom 4. März 1813, AN, AF IV 1840 .

398 Protokoll des Verhörs vom 5. Januar 1811, HStAD, GB, Nr. 10899. 
Der Maire von Datteln und Waltrop beschwerte sich über den nächtlichen Besuch von Zolldienern in seinem Haus ${ }^{399}$ und prangerte darüber hinaus grundsätzlich das willkürliche Vorgehen der Zolldiener an. Diese Hausvisitationen, kritisierte er Ende August 1812, hören jetzt auf, gesetzliche Handlungen zu seyn und sind [...] zu tumultuarischen Gewalthätigkeiten ausgeartet ${ }^{400}$. Der Advokat Wardenburg beantragte im Herbst 1813 die Entschädigung von Anna Catharina Henrichs pour detention arbitraire et illegale dépuis le 15 Janvier 1813 jusqu'au 29 Avril de la même année durch den Prokurator Sybel ${ }^{401}$. Aus Bensberg gingen im Frühjahr 181329 Reklamationen von Einwohnern ein, die mit den Aufständischen zugleich verhaftet worden waren ${ }^{402}$.

Bei den einheimischen Staatsdienern fanden die Betroffenen durchaus Gehör und Unterstützung für ihre Anliegen. Im Fall Wedel beispielsweise waren sich die Düsseldorfer Polizeibehörde und der Präfekt darin einig, daß es sich um einen Willkürakt handele, der hart zu ahnden sei. Nach Auffassung Borckes ließ sich das Vorgehen des Zolls durch nichts rechtfertigen, weil für ihn das Haus eines Bürgers eine heilige Freistätte darstellte - womit er auf den Gesetzestext anspielte - und durch gesetzlichen Mißbrauch bestürmt worden war ${ }^{403}$. Innenminister Nesselrode teilte die Auffassung des Präfekten. Auch er lehnte nächtliche Visitationen kategorisch $a^{404}$. In der Hausdurchsuchung des Maires von Datteln und Waltrop sah Borckes Nachfolger im Rheindepartement eine Schikane des Zolldieners. Wie habe man glauben können, meinte Spee Anfang Oktober 1812, der Grundbesitzer Boehne, dessen Einkünfte sich auf eine Höhe von 50000 Francs beliefen, beteilige sich am Schmuggel $^{405}$. Er hielt vor diesem Hintergrund die Bestrafung derjenigen Douaniers für notwendig, die derartige Übertretungen begingen.

Die einheimische Staatsdienerschaft verurteilte ebenfalls die Konfiskation von Waren.

Man beklage sich, kritisierte der Präfekt des Emsdepartements in seinem Reisebericht vom 11. August 1810, über die saisies subites et inopinées de marchandises, dont l'entrée n'avoit été defendue par aucune loi promulguée dans le Grand-Duché de Berg. Ces saisies ont été faites d'une manière tumultuaire sans aucune des formes que les loix françaises ont introduites pour la garantie des droits de paisibles citoyens et cependant c'étoit les loix françaises que les douaniers français annonçoient être chargés d'appliquer au Grand-Duché. [...]

399 Seine Klage wurde am 28. Dezember 1812 vom Spezialtribunal der Zölle abgewiesen, HStAD, GB, Nr.11027.

400 Beschwerdebrief des Maires an den Unterpräfekten vom 29. August 1812, ibid.

401 Antrag des Advokaten Wardenburg vom 17. Oktober 1813, HStAD, GB, Nr. 6392.

402 Schreiben Sethes an Roederer vom 10. April 1813, AN, AF IV 1840.

${ }^{403}$ Borcke in seinem Schreiben an den Hofratspräsidenten Ritz vom 17. Oktober 1810, HStAD, GB, Nr.11027.

404 Beugnot machte dies in seinem Schreiben an Nesselrode vom 9. November 1810 deutlich, HStAD, GB, Nr. 6305.

405 Schreiben des Präfekten Spee an Beugnot vom 1.Oktober 1812, HStAD, GB, Nr. 11027. 
J'ajoute que le renvoi des causes rélatives à ces saisies au conseil de prises séant à Paris, Tribunal éloigné d'au délà de 150 lieues de plusieurs communes de mon Departement, et dont les loix et la forme de procédure ne sont connues de personne dans le Departement, le rend très difficile aux habitans de ce departement d'obtenir la justice qu'ils croient pouvoir reclamer [sic] ${ }^{406}$.

Einheimische Amtsträger verhielten sich besonders streng gegenüber solchen Bediensteten, denen Körperverletzungen zur Last gelegt wurden. Schrauben, verurteilte Präfekt Borcke das Vorgehen des Barmer Polizeikommissars De Witt, sind wirkliche peinliche Instrumente, deren Anwendung mittelst Quetschung des Daumens biß zum unerträglichsten Schmerz getrieben werden und die völlige Lähmung dieses Gliedmaßes zur Folge haben kann. Früher hätten diese als Folterinstrumente gedient ${ }^{407}$.

Solche Vexationen, solche gröbliche Excesse, kritisierte der Kaiserswerther Maire Herschbach am 14. September 1812 den Douanier, der einen Jungen geschlagen hatte, - wenn auch dieses blos Folgen eines besinnungslosen $\mathrm{Zu}$ standes waren - sind wahrlich nicht geeignet, um ein Abgaben-System beliebt und erträglich zu machen, das in den Augen der Menge bereits aus dem verkehrtesten Gesichtspuncte betrachtet wird, und müssen das Volk zur Erbitterung gegen die subalternen Zoll-Préposés reitzen ${ }^{408}$.

Ein Staatsdiener, der wiederholt für die Einwohner Partei ergriff und sich für die Wahrung ihrer Rechte engagierte, war Generalprokurator Sethe. Um beispielsweise den Ausschreitungen von Zolldienern Einhalt zu gebieten, ließ er im Herbst 1812 beim Finanzminister anfragen, ob man diesen nicht empfehlen könne, von ihren Waffen nur bei Notwehr Gebrauch zu machen ${ }^{409}$. Nach Niederschlagung des Aufruhrs Anfang Februar 1813 versuchte Sethe, Licht in das Vorgehen der Gendarmerie während der Unruhen zu bringen, zumal die Gendarmen offensichtlich damit fortfuhren, Verdächtige zu verhaften ${ }^{410}$. Zu diesem Zweck wandte er sich am 4. Februar 1813 an die Gendarmerie und forderte von ihr Informationen über die Verhaftungen sowie die dazugehörigen Protokolle. Nachdem der Kapitän und Kommandant der Gendarmerie, von Krane, am 7. Februar 1813 jegliche Stellungnahme verweigert hatte, weil es sich für ihn allein um eine Militärangelegenheit handelte,

406 So der Präfekt des Emsdepartements in seinem Reisebericht vom 11. August 1810, HStAD, GB, Nr. 4400 .

407 Borcke in seinem Schreiben an den Unterpräfekten Schleicher vom 25.Juni 1811, HStAD, GB, Nr.10899. De Witt gestand in seiner Vernehmung das Schrauben zu, verteidigte sich indes damit, den Beklagten an der Flucht gehindert, aber nicht zum Geständnis gezwungen zu haben. Außerdem wies er darauf hin, daß der Gebrauch von Schrauben auch in der Gendarmerie üblich sei. Vgl. das Protokoll der Vernehmung des Polizeikommissars durch den Richter Schramm vom 29. Mai 1811, ibid.

408 Der Kaiserswerther Maire Herschbach in seinem Schreiben an den Präfekten Spee vom 14. September 1812, HStAD, GB, Nr. 11027.

409 Schreiben Beugnots an Sethe vom 25. September 1812, ibid.

410 Schreiben Sethes an den Oberst der Gendarmerie vom 4. März 1813, AN, AF IV 1840. 


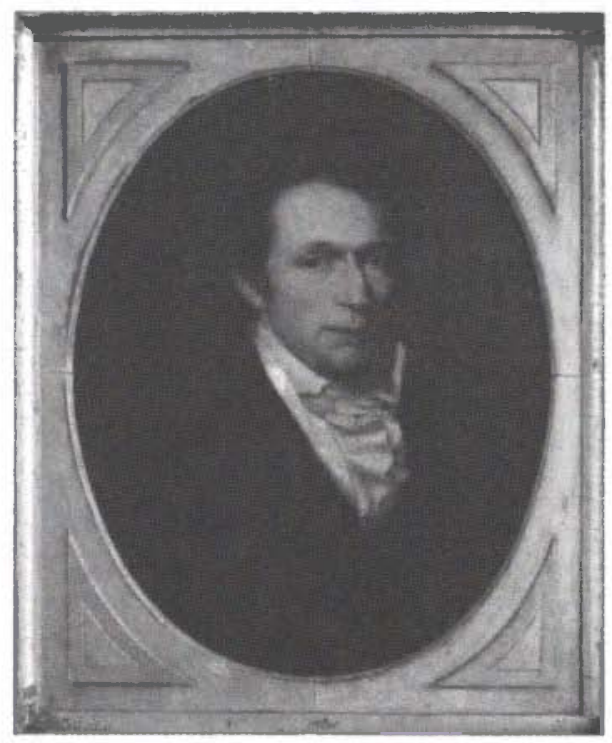

Abb. 5: Christoph Wilhelm Henrich Sethe (1765-1855).

Quelle: Preußen-Museum NRW, Wesel.

forderte Sethe Krane am 8. Februar 1813 erneut dazu auf, Bericht zu erstat$\operatorname{ten}^{411}$.

Da Krane sich auch weiterhin nicht zu den Vorwürfen äußerte, die der Gendarmerie zur Last gelegt wurden, wandte sich der Generalprokurator am 4. März 1813 direkt an den Oberst der Gendarmerie, Graf von Goltstein, und teilte diesem mit, die Gendarmerie habe gegen die Bestimmungen verstoßen, die die Freiheit der Untertanen sicherten, und nehme weiterhin unberechtigte Verhaftungen vor. Sethe drohte, dieses Vorgehen gesetzlich streng zu ahn$\mathrm{den}^{412}$. Als sich herausstellte, daß Lemarois am 12. Februar 1813 dem Kolonel der Gendarmerie eine Blankovollmacht erteilt hatte, wonach alle Personen von der Gendarmerie verhaftet werden und bis auf weiteres in Haft bleiben sollten, die an den Unruhen teilgenommen hatten oder der Teilnahme verdächtigt wurden, ging der Generalprokurator noch weiter.

Schon früherhin, teilte er Goltstein am 7.März̄ 1813 mit, habe ich zu bemerken Gelegenheit gehabt, daß die Gendarmerie Arrestationen verfügte, wozu sie nach den Gesetzen nicht autorisirt ist; und es war schon längst mein Vorhaben Sie auf diese Irregularitäten und die fehlerhafte Uebersetzung in dem $\$ 4, N .4$ der Verordnung vom 22. Nov. $1808^{413}$ aufmerksam zu machen, welche hauptsächlich

411 Schreiben Sethes an den Gendarmerie-Kapitän Krane vom 4. Februar 1813; Antwortschreiben Kranes an Sethe vom 7.Februar 1813; erneutes Schreiben Sethes an den Gendarmerie-Kapitän vom 7. Februar 1813, ibid.

412 Schreiben Sethes an den Oberst der Gendarmerie vom 4. März 1813, ibid.

413 Demnach war die Gendarmerie verpflichtet, alle auf frischer That ertappte[n], oder eines begangenen Verbrechens verdächtige[n] Personen zu arretiren, wobei der Ausdruck »eines begangenen Verbrechens verdächtige Personen« fälschlicherweise als Übersetzung für 
den Irrthum bey der Gendarmerie veranlaßt zu haben scheint. Andere überhäufte Arbeiten haben dies bisher verzögert. Jetzt, wo diese Arrestationen sich in einem hohen Grad häuften; wo nach gedämpftem Aufruhr noch viele Individuen fortwährend, ohne alle legale Form, verhaftet, und ihrer Freiheit beraubt werden, ohne daß der Verdacht eines von ihnen begangenen Verbrechens gehörig constatiret worden; wo die Gefängnisse mit diesen Gefangenen so angefüllt werden, daß sie solche nicht mehr fassen können und gefährliche ansteckende Krankheiten darin entstanden sind; jetzt dürfte ich, ohne mich verantworlich zu machen, nicht länger anstehen, [...] die Herren Procureurs bey den Tribunälen ihr Amt wahrnehmen, oder eine vorherige freundschaftliche Erinnerung vorangehen zu laßen. [...] Da die Tribunäle und das bey denselben fungirende Offentliche Ministerium verpflichtet sind, sich den Gesetzen zu conformiren und keine andere Instruction als diese erhalten haben, so werden Sie, Herr Oberster, selbst ermessen, was für Collisionen und Verwirrungen daraus entstehen müßen, wenn auf der einen Seite die Tribunäle und Procureurs genau nach der gesetzlichen Vorschriften sich richten, und auf der andern Seite die Hülfsbeamte der gerichtlichen Polizey ganz davon abweichend und in gesetzlosen Form verfahren. Es ist daher die höchste Zeit, daß der großen Unordnung, welche in die Ausübung der gerichtlichen Polizey eingeflossen ist, ein Ende gemacht, und die gesetzliche Ordnung darin wieder hergestellet werde ${ }^{414}$.

Neben der Gendarmerie informierte Sethe ebenfalls seinen Vorgesetzten in Düsseldorf von den gegen die Gendarmerie vorliegenden Beschwerden. Am 6. Februar 1813 teilte er Nesselrode mit, viele der Revoltierenden, die an verschiedenen Stellen schwere Verbrechen begangen hätten, seien durch die Gendarmerie und das Militär aufgegriffen worden. Cependant, bemängelte er in diesem Kontext, ces insurgés n'ont pas éte traduits comme l'ordre prescrite l'exige, aux Procureurs des Tribunaux, afin que ceux-ci puissent exercer leur ministère contre ces criminels ${ }^{415}$. Am 15. Februar 1813 informierte der Generalprokurator den Innenminister darüber, da $\beta$ viele Reklamationen über unrechtmäßige Verhaftungen in Bensberg eingegangen seien. Es sehe so als, schloß Sethe daraus, als ob die Verhaftungen in Bensberg nicht mit genügend Vorsicht, sondern voreilig vorgenommen worden wären. Selbst wenn es während der Unruhen nicht möglich gewesen wäre, den Unschuldigen vom Schuldigen zu trennen, hätte man doch nach Beendigung der Proteste eine Liste mit den festgenommenen Personen und den jeweiligen Verhaftungsgründen anfertigen müssen. Das wäre in Bensberg um so notwendiger gewesen, als man hier für die Verhaftungen in die Häuser eingedrungen sei und Eigentumsrechte der Bewohner verletzt habe ${ }^{416}$.

personne poursuivies par la clameur publique gewählt worden war. Vgl. Nesselrodes Verordnung wegen der für das Großherzogthum errichteten Gendarmerie vom 22 . November 1808, \$4, Nr.4, HStAD, Depositum Stadt Ratingen 56.

414 So Sethe in seinem Schreiben an Goltstein vom 7. Februar 1813, AN, AF IV 1840.

415 Schreiben Sethes an Nesselrode vom 6. Februar 1813, ibid.

416 Schreiben Sethes an Nesselrode vom 15. Februar 1813, ibid. 
Die Rechtswidrigkeiten, die den Amtsträgern zur Last gelegt wurden, zogen unterschiedliche Folgen nach sich. Eine Anzahl von Staatsdienern wurde für die Verletzung der Dienstpflicht konkret belangt. Beispielsweise verlor der Mülheimer Polizeikommissar Guilleaume auf Gutachten des Staatsrats sein $\mathrm{Amt}^{417}$. Für den flüchtigen Lieutnant der Douanen, Lohrmann, der angeklagt war, im Kanton Gummersbach einen Schmuggler erschossen zu haben, verhängte das Spezialtribunal der Zölle am 10. Juli 1813 eine Kerkerstrafe ${ }^{418}$. Gendarmen, die aus Trunkenheit oder Liederlichkeit ihre Aufgaben nicht erledigt hatten oder auffällig geworden waren, wurden häufig in ihre alten Positionen zurückversetzt oder zur Strafe in die Infanterie geschickt ${ }^{419}$. In Mülheim mußte ein Gendarmerieoffizier demgegenüber eine achttäge Haft absitzen ${ }^{420}$. Der Zolleinnehmer Thiebes wurde vom Hofrat zu einer Geldstrafe verurteilt. Darüber hinaus sollte er sich bei dem Mann, den er beleidigt und verletzt hatte, für sein Verhalten entschuldigen ${ }^{421}$.

Andere Dienstvergehen oder Rechtsverletzungen blieben dagegen ungeahndet. Der Soester Polizeikommissar Martin etwa kam glimpflich davon. Er wurde zur Mäßigung bei der Ausübung seiner Amtsgeschäfte angehalten, ging aber ansonsten straffrei aus. Obwohl die Regierungsbeamten Martins Vorgehen durchaus als rechtswidrig betrachteten, heiligte der Zweck ihrer Meinung nach die Mittel. Sie sahen in der Züchtigung des Gefangenen ein taugliches und notwendiges Zwangsmittel zur Aufrechterhaltung der öffentlichen Sicherheit ${ }^{422}$. Die Klage des Maires von Datteln und Waltrop wurde ganz zurückgewiesen. Der Zoll rechtfertigte die Hausdurchsuchung mit dem Argument, Boehne habe bei den Debitanten seiner Mairie schon seit längerem kein Salz mehr abgenommen ${ }^{423}$.

Die Staatsdiener waren sich in der Frage, wie die Eingriffe in geschützte Rechtsbereiche zu beurteilen und welche Konsequenzen daraus zu ziehen waren, nicht immer einig. Gelegentlich kam es sogar zu scharfen Auseinandersetzungen. Ernsthafte Konflikte entfachten zum einen über die Einschätzung der Vorgänge im Zollwesen. Während die einheimischen Beamten für die Ereignisse und Entwicklungen im Schleichhandel in erster Linie die Zolldiener verantwortlich machten, suchte Finanzminister Beugnot die Hauptschuld bei

417 Beschluß des Innenministers vom 11. April 1809, HStAD, GB, Nr. 4481.

418 Siehe den Monatsbericht Beugnots an Roederer für August 1812, AN 29 AP 39, ferner den Vortrag Bislingers in der Staatsratssitzung vom 10. Juli 1813, HStAD, GB, Nr. 313.

419 Viele Gendarmen kamen vom linken Rheinufer oder aus Frankreich und hatten vielfach zuvor in der Armee gedient, wie folgende Akte verdeutlicht: Diejenigen Gendarmen, welche wegen unanständigen Betragens oder schlechten Lebenswandels entweder gänzlich entlassen oder zur Infanterie oder Cavalerie versetzt sind, HStAD, GB, Nr. 6197.

420 Beschluß des Innenministers vom 11. April 1809, HStAD, GB, Nr. 4481.

421 Urteil des Hofrats vom 21. Juni 1811, HStAD, JB, Hofrat A, Nr.91.

422 Vortrag von Fuchsius im Staatsrat vom 7. Mai und 19. August 1812, HStAD, GB, Nr.319.

423 Schreiben des Zollverwalters vom 3. Oktober 1812; Entscheidung des Spezialtribunals der Zölle vom 28. Dezember 1812, HStAD, GB, Nr. 11027. 
den Einwohnern. Zwar stritt er nicht ab, daß die von den Douanen begangenen Handlungen widerrechtlich waren. Doch gab es seiner Meinung nach berechtigte Gründe für das Vorgehen der Zollbediensteten. Je ne prétends pas excuser, gestand er beispielsweise im Fall Wedel, le zèle assurément mal entendu que cet employé a déployé, même depuis que je suis instruit des motifs qui le faisaient agir ${ }^{424}$. Doch sei das Vorgehen des Düsseldorfer Douaniers gerechtfertigt, weil der französische Zoll den Fischer verdächtigt habe, an einer Schmuggelaktion beteiligt zu sein. Die Schleichhändler hätten ihren Plan letztlich durchführen können, weil der Zoll an der Erledigung seiner Aufgabe gehindert worden sei.

Dans le fait l'exportation frauduleuse s'est effectuée comme à force ouverte, schrieb der kaiserliche Kommissar. Les douaniers français n'ont pu s'y opposer autrement qu'en lâchant des coups de fusil qui ont mis deux hommes à bas; mais la marchandise a passé. Des deux hommes atteints l'un est mort, l'autre brièvement blessé; et on serait tenté de regretter que le Sr. Bohr eut été interrompu même dans ses procédés irréguliers, s'ils avaient dû prévenir un évènement aussi déplorable $e^{425}$.

Man müsse gestehen, verteidigte Beugnot die seiner Obhut anvertrauten Zolldiener gegenüber Spee am 11.Juni 1812, daß wenn man dieselben überall wie feindliche Wesen betrachtet, bey jeder Gelegenheit mit Drohungen und Schmähungen überhäuft, sie nothwendig zu immer größeren Excessen müssen geneigt werden. Die gesellschaftliche Ordnung zu stören, sich ohne Grund mit den Einwohnern zu überwerfen, liegt offenbar nicht im Interesse dieser Zollbedienten, besonders da jene ihnen an der Zahl immer sehr weit überlegen sind, auch würden sie sicher manchen Exceß nicht begangen haben, wenn sie unangetastet für ihre eigene Person, überall jene Aufnahme gefunden hätten, welcher jeder von der Regierung zum gemeinen Besten angestellter Beamte mit Recht zu erwarten hat ${ }^{426}$.

Beugnot lehnte es ab, Zolldiener am Gebrauch ihrer Waffe zu hindern, denn seiner Meinung nach benötigten sie diese zur Vertheidigung ihres Leibes und ihres Lebens ${ }^{427}$. Ebensowenig hielt er die Bestrafung des Zolls für gerechtfertigt. Im Fall Wedel ging er sogar so weit, von Nesselrode mehr Protektion und damit mehr Handlungsspielraum für die Douanen zu verlangen ${ }^{428}$. Fortan sollten Zolldiener nur noch auf Befehl des Finanzministers verhaftet werden können.

Nesselrode vertrat eine andere Auffassung als sein Kollege im Finanzministerium. Er verurteilte das willkürliche Vorgehen der Zolldiener gegen den Fischer. Dennoch kam er der Aufforderung Beugnots nach und machte die

425 Ibid.

426 Schreiben Beugnots an Spee vom 11. Juni 1812, HStAD, GB, Nr. 11027.

427 Schreiben Beugnots an Sethe vom 25. September 1812, ibid.

428 Schreiben Beugnots an Nesselrode vom 9. November 1810, HStAD, GB, Nr.6305. 
Festnahme der Zolldiener künftig vom Plazet des Finanzleiters abhängig. Er rechtfertigte diesen Schritt mit der Notwendigkeit, das Großherzogtum Berg an die innerfranzösischen Zustände anzupassen. J'en trouve, begründete er, des motifs suffisans [sic] dans l'analogie du service des employés des préposés français, d'où doit suivre comme conséquence naturelle, l'adoption des mêmes mesures de garantie ${ }^{429}$.

Für Konfliktstoff unter den Staatsdienern sorgte zum anderen Generalprokurator Sethe mit seinem Schritt, die Gendarmerie für ihr Verhalten während der Unruhen zur Rechenschaft zu ziehen. Innenminister Nesselrode verurteilte Sethes Vorgehen und wies ihn umgehend in die Schranken seiner Funktionen zurück. Ohne auf die Anklagepunkte selbst einzugehen, bezeichnete er Sethes Versuch, die Gendarmerie in die Verantwortung zu nehmen, als Amtsanmaßung.

Er sei wirklich erstaunt, rügte er den Generalprokurator am 19. Februar 1813, daß Sethe die Angelegenheit so falsch bewertet habe. Il ne s'agit pas d'un service ordinaire de gendarmerie, mais d'une véritable opération militaire qui a eu lieu contre les insurgés et dont Mr. le Capitaine de Krane comme militaire a été chargé du commandement par le ministère de la guerre parce qu'on l'a tenu le plus propre pour diriger cette opération [...]. C'est n'est donc qu'au ministère de la guerre que la colonne militaire mobile envoyée à cet effet, et qui non seulement était composée de gendarmerie mais aussi d'infanterie et de cavalerie, avait à rendre compte de ses opérations ${ }^{430}$.

Ohne die Antwort aus Düsseldorf abzuwarten, hatte Sethe unterdessen bereits eine weitere Verfügung angeordnet. Am 15. Februar 1813 hatte er den Prokuratoren der erstinstanzlichen Gerichte mitgeteilt, daß die Anzahl von Reklamationen fälschlicherweise Verhafteter und Freigelassener darauf schließen lasse, daß die Gendarmerie bei den Verhaftungen ohne Regeln und Formalia vorgegangen sei und ihre Pflichten als Justizpolizei vernachlässigt habe. Deshalb forderte er sie dazu auf, de constater par le moyen des autorités locales et d'autres témoins dignes de foi, la manière dont on a procédé aux arrestations dans votre arrondist. et de vérifier si la gendarmerie a observé les règlemens [sic], si elle a employé ses armes mal à propos, blessé des individus sans nécessité ou commis d'autres excès ${ }^{431}$.

Die Hinzuziehung der Gerichtsbehörden hatte für Sethe ein politisches Nachspiel. Anstoß dazu gab ein Zirkular, das der Substitut des Öffentlichen Ministeriums am Arrondissementgericht von Düsseldorf und Elberfeld, Carl Fuchsius, in Reaktion auf Sethes Schreiben am 19. Februar 1813 an die Friedensrichter von Düsseldorf und Elberfeld richtete. Darin teilte er ihnen mit,

429 Schreiben Nesselrodes an Beugnot vom 9. November 1810, ibid.

430 Schreiben des Innenministers an den Generalprokurator vom 19. Februar 1813, AN, AF IV 1840.

431 Schreiben Sethes an die Prokuratoren der erstinstanzlichen Gerichte vom 15. Februar 1813 , ibid. 
die Gendarmerie habe ihre Pflichten vollständig verletzt. Gleichzeitig forderte er sie dazu auf, ihm alle Informationen über das Vorgehen der Gendarmerie zukommen zu lassen ${ }^{432}$. Eine Kopie des Zirkulars geriet direkt in die Hände Beugnots, der es umgehend an Roederer weiterleitete.

Ohne den Hergang des Vorfalls genauer zu kennen, ordnete der MinisterStaatssekretär daraufhin am 2. April 1813 an, Fuchsius wegen Hochverrats sofort zu suspendieren ${ }^{433}$. Im nachhinein stellte sich jedoch heraus, da $B$ das Zirkular nicht auf die Initiative des Substituten zurückging, sondern von Generalprokurator Sethe selbst veranlaßt worden war ${ }^{434}$. Fuchsius wurde entlastet, und die Angelegenheit erhielt nicht nur eine neue Wendung, sondern auch eine andere Dimension: Das Verhalten der Gendarmen wurde durch das Vorgehen des Generalprokurators überlagert. Der Kläger wechselte auf die Anklagebank. Roederer wandte sich daraufhin an den französischen Justizminister und bat ihn um seine Stellungnahme in der Angelegenheit. Er wollte von Regnier zum ersten erfahren, ob der Substitut wieder in seine alten Funktionen gesetzt werden könne, zum zweiten, ob der Prokurator des erstinstanzlichen Gerichtes durch den ersten Brief Sethes entschuldbar sei, zum dritten, ob Sethe eine Geld- oder eine Disziplinarstrafe erhalten solle oder gar noch schärfer zu bestrafen sei, und zum vierten, welches generell die Disziplinarstrafen für höhere Beamte seien ${ }^{435}$.

Was den eigentlichen Tatbestand betraf, waren sich die französischen Staatsbeamten einig. Ihrer Ansicht nach hatte Sethe auch in dieser Sache nicht die politischen Funktionen seines Amts erfüllt. Über die Motive und Ursachen, die den Generalprokurator zu seinem Vorgehen bewogen hatten, wie auch über die daraus zu ziehenden Konsequenzen gingen die Meinungen der Regierungsbeamten jedoch auseinander. Für Regnier führte kein Weg an der Entlassung des Generalprokurators vorbei. Die Tatsache, daß Sethe die Sache der Gendarmerie als Angelegenheit der einfachen Justizpolizei betrachtet hatte, machte seiner Meinung nach deutlich, daß der Generalprokurator seinen Funktionen nicht gewachsen und gerade in schwierigen Zeiten politisch unzuverlässig war. Falls man ihn dennoch weiterhin einzustellen gedachte, sollte man ihm ein weniger anspruchsvolles Amt übertragen ${ }^{436}$.

Roederer hielt es demgegenüber nicht für erforderlich, Sethe seines Amts $\mathrm{zu}$ entheben. Zwar glaubte auch er, daß der Generalprokurator aufgrund sei-

432 Schreiben des Substituten an die Friedensrichter Elberfelds und Düsseldorfs vom 19. Februar 1813, ibid.

433 Das geht aus Nesselrodes Schreiben an Roederer vom 7. April 1813 hervor, AN, 29 AP 39.

434 Schreiben Beugnots an Roederer vom 7. April 1813, AN, 29 AP 38; Schreiben Nesselrodes an Roederer vom 7. April 1813, ibid.

435 Brief Roederers an den französischen Justizminister vom 14. April 1813, AN, AF IV 1840.

436 Antwortschreiben des französischen Justizministers an den Minister-Staatssekretär vom 2. Mai 1813, ibid. 
ner preußischen Herkunft und seiner Einflußmöglichkeiten auf die Gerichte einen politischen Unsicherheitsfaktor darstellte. Doch sah er in dem bisherigen Verhalten Sethes keinerlei Anzeichen für politische Untreue. Jusqu'à présent, erläuterte Roederer gegenüber Napoleon am 3. Mai 1813, je ne vois rien qui autorise à le soupçonner de trahison; [...] c'est un homme consciencieux qui tient à un serment et à la loi ${ }^{437}$. Auch in Sethes Einschreiten nach den Unruhen sah der Minister-Staatssekretär keinerlei Indiz für Hochverrat. Sethe sei un caractère sans force et sans élévation, meinte er statt dessen, qui a cru avoir besoin de se populariser quand il a vu la révolte apaisée; d'ailleurs esprit allemand qui sort difficilement des règles ordinaires des tribunaux et homme de robe entiché de sa prérogative sur le militaire, et de son autorité sur la gendarmerie $^{438}$. Darüber hinaus meinte Roederer, Sethe sei sich weder der Militäraufgaben der Gendarmerie noch der politischen Funktionen des ihm verliehenen Amts bewußt gewesen.

Il a paru fort surpris, erläuterte er Napoleon, lorsque je lui ai présenté le service de la gendarmerie comme un service mixte; tantôt judiciaire et tantôt militaire; et indépendant de lui sous ce dernier rapport. Il n'a pas été moins étonné quand je lui ai représenté que le ministère d'un procureur général n'était pas purement judiciaire; qu'il participait à l'administration, qu'il devait être exercé suivant l'esprit du gouvernement dans les affaires publiques; principes que Mr. Beugnot a peut être [sic] à se reprocher de ne pas lui avoir inculqués dans les communications habituelles qu'il devrait entretenir avec les principaux magistrats ${ }^{439}$.

Beugnot brachte die Vorgänge ebenfalls nicht in Verbindung mit den persönlichen Bindungen Sethes zu Preußen, sondern führte das Verhalten des Generalprokurators in erster Linie auf dessen mangelnde Vertrautheit mit den politischen Funktionen seines Amts zurück. Auch er hielt es deshalb für ausreichend, Sethe darauf hinzuweisen, sein Amt in Zukunft besser mit dem politischen Auftrag in Einklang zu bringen, der daran geknüpft war ${ }^{440}$. Ob es überhaupt personelle Alternativen zu Sethe gab, ist ohnehin zweifelhaft.

Rückendeckung erhielt Sethe im nachhinein ebenfalls aus dem Innenministerium. Nesselrode setzte sich am 7. April 1813 dafür ein, Sethe mit Nachsicht zu behandeln. Seiner Meinung nach hatte der Generalprokurator voreilig gehandelt und zudem den Gesetzestext zu streng genommen bzw. irrtümlich auf eine Angelegenheit des Militärs und der Hohen Polizei angewandt. Darüber hinaus verwies Nesselrode auf die großen Verdienste des Generalprokurators bei der Neuordnung der Justiz ${ }^{441}$.

Sethe selbst führte die gegen ihn erhobenen Anschuldigungen auf eine Verkettung ungünstiger Umstände zurück. Ihm zufolge war das Zirkular des Sub-

\footnotetext{
437 Bericht Roederers an Napoleon vom 3. Mai 1813, AN, AF IV 1226.

438 Ibid.

439 Vgl. ibid.

440 Schreiben Beugnots an Roederer vom 10. Juli 1813, AN, AF IV 1840.

441 Schreiben Nesselrodes an Roederer vom 7. April 1813 AN, 29 AP 39.
} 
stituten zu Unrecht in die Hände der Gendarmerie gelangt und von dieser darüber hinaus falsch interpretiert worden. Außerdem verteidigte er sich damit, nicht darüber informiert worden zu sein, daß sich das Land im Kriegszustand befand - womit er der Regierung abermals indirekt den Ball zuspielte.

Ein Kriegs- bzw. Ausnahmezustand, erklärte er, n'est pas à présumer; il doit etre déclaré, et on aurait au moins dâ m'en informer. Mais je n'ai reçu aucunes communications là dessus. Je n'avais aucune notion que les expéditions de la gendarmerie dûssent etre considérées comme des expéditions militaires dans un pays étant en état de guerre et hors les lois. Aussi les suites qu'on a donné [sic] aux expéditions de la gendarmerie ne se conforment pas à cette manière de voir. S'il y avait un état de guerre on aurait dû terminer l'affaire militairement. Mais on a renvoyé ceux qui ont été pris par la gendarmerie aux tribunaux ordinaires \& on a laissé procéder les officiers de la police judiciaire contre les insurgés dans les formes ordinaires ${ }^{442}$.

Was den Inhalt der Vorwürfe gegen die Gendarmerie anbelangte, stellte sich im nachhinein heraus, daß diese gerechtfertigt waren. Offensichtlich hatten die Gendarmen die allgemeine Verunsicherung im Land in der Tat dazu genutzt, Jagd auf Deserteure, Refraktäre, Landstreicher und Vaganten zu machen.

Er entnehme Beugnots Schreiben vom 4.Juni 1813, teilte Roederer dem kaiserlichen Kommissar am 11. Juni 1813 mit, que M. Krane n'a éprouvé de résistance nulle part, qu'il a fait des courses de police, qu'il a ramassé des mendians et des trainards et que les batailles d'Elberfeld et de Bensberg n'ont existé que dans les faux rapports par les quels il a cherché à se faire valoir. Si M. Krane a osé abuser à ce point de la confiance des ministres, il est probable qu'il n'a pas été scrupuleux envers les habitans [sic] et qu'il a pu se permettre des vexations et des irrégularités qui justifieraient ou qui excuseraient du moins la démarche du procureur général ${ }^{443}$.

Da Sethe augenscheinlich seine Amtsfunktionen nicht mißbraucht hatte, lehnte Roederer Sanktionen gegen den Generalprokurator ab, zumal er in dem zweimonatigen Aufenthalt Sethes in Paris eine ausreichende Bestrafung

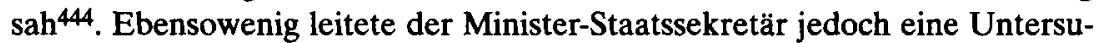
chung gegen die Gendarmerie ein. In der Sache blieb der Generalprokurator somit erfolglos. Sein Versuch, den Staat auf die Einhaltung der Gesetze zu verpflichten, scheiterte. Die Regierung brachte unmißverständlich zum Ausdruck, daß sie dem Staatsinteresse Vorrang vor dem Recht gab und die von ihr kodifizierten Rechte nicht als unantastbar betrachtete. Im Unterschied zum Fall des Gummersbacher Friedensrichters Pollmann schlugen auch Sethes Bemühungen fehl, die Rechtsprechung als Kontrollorgan und Rechtsgaranten

442 Undatiertes Exposé sur le Circulaire du Procureur du tribunal de $1^{\text {ere }}$ Instance de Dusseldorf, ou plutôt sur ma lettre du 15 fevrier adressé au d. Procureur (Mai 1813), AN, AF IV 1840.

443 Schreiben Roederers an Beugnot vom 11.Juni 1813, ibid.

444 AN, 29 AP 37. 
ins Spiel zu bringen. Dieses Mal ließ sich die Regierung von der Justiz keine Zügel anlegen. Sie nahm im Gegenteil für sich das Recht in Anspruch, die Justiz zu beaufsichtigen und politisch in die Verantwortung zu nehmen.

$\mathrm{Da} ß$ die Behörden und in letzter Instanz die Regierung Verstöße gegen geltendes Recht, welche von Mitgliedern der staatlichen und lokalen Funktionsträgerschaft begangen wurden, mit Blick auf Staatsinteressen tolerierten oder gar rechtfertigten, wirkte sich nachhaltig auf die Stabilität der Rechtsordnung aus. Rechte waren weder garantiert noch beständig. Ebenso waren die Rechtsfolgen nicht vorhersehbar oder kalkulierbar. Vor allem waren sie nicht für alle gleich, weil vergleichbare Tatbestände nicht einheitlich klassifiziert und auch nicht einheitlich geahndet wurden. Unterschiede wurden zwischen öffentlichen Amtsträgern und noch mehr zwischen Einwohnern und Staatsdienern gemacht. Die Entwicklungen im Schmuggel haben dokumentiert, wozu diese mehrgleisige Politik schlimmstensfalls führen konnte.

Es war vor allem das Verdienst der einheimischen Staatsdiener, daß Staat und Gesellschaft hierbei nicht vollständig auseinandertraten ${ }^{445}$. Während die französische Regierung staatliche Eingriffe in geschützte Rechtsbereiche politisch legitimierte, deckten oder rechtfertigten einheimische Bedienstete durch Indifferenz oder Ignoranz, Parteinahme oder Komplizenschaft umgekehrt oftmals rechtswidrige Handlungen der Untertanen. Damit förderten auch sie die Aufweichung bestehender Normen, sorgten aber gleichzeitig für einen Ausgleich zwischen Herrschafts- und Gesellschaftsinteressen und für ein Stück Rechtssicherheit vor Ort ${ }^{446}$.

\subsubsection{Entwicklungen im Jahre 1813}

Trotz ihrer Mittlerfunktion vermochten die einheimischen Amtsdiener nicht, den beschleunigten Abbau an Rechtsschutz zu verhindern, der durch die Proteste zu Beginn des Jahres 1813 ausgelöst wurde und Ausdruck bzw. Folge der schwindenden Autorität sowie des wachsenden Sicherheitsbedürfnisses der französischen Herrschaft war. Der Rückgang an Rechtssicherheit ließ sich zum einen an der verstärkten Überwachung des Großherzogtums ablesen. Französische Spione durchquerten vermehrt das Land, um Einblick in die Gesinnung der Einwohner und Staatsdiener zu erhalten und Anzeichen von Op-

445 Das galt im übrigen auch für die Laienrichter der Schwurgerichte.

446 Beispielsweise stellte sich der Duisburger Maire Speck im Frühjahr 1811 der übereilten Verhaftung eines französischen Refraktärs durch den Rittmeister Lampinet in den Weg. Bericht des Rittmeisters und Kommandanten der Gendarmerie im Rheindepartement, Lampinet, an den Präfekten Borcke vom 11.Juni 1811, HStAD, GB, Nr.11144. Die Lokalbehörden von Elberfeld und Lüttringhausen weigerten sich 1811, dem Befehl der französischen Gendarmerie Folge zu leisten, französische Refraktäre, die angeblich in den dort angesiedelten Fabriken arbeiteten, auszuliefern. Sie forderten Beweise für den Verdacht, daß die Fabriken den Konskribierten als Refugium dienten. Vgl. das Schreiben Marets an Roederer vom 8.Juli 1811, den Brief, den Roederer daraufhin am 10. Juli 1811 an Nesselrode sandte, sowie das Antwortschreiben Nesselrodes an Roederer vom 17.Juli 1811, AN, AF IV 1874. 
position aufzuspüren ${ }^{447}$. Fliegende Gendarmerieeinheiten machten zunehmend Jagd auf Deserteure, Refraktäre und Vaganten. Presse, Vereine und Gesellschaften, die seit dem Aufruhr unter Verschwörungsverdacht standen, wurden stärker als zuvor beaufsichtigt. Die Bewilligung neuer Gesellschaften zögerte man sogar ganz hinaus.

On attribue en partie la fermentation qui regne dans le Grand Duché, au grand nombre de clubs ou de sociétés qui se rassemblent sous divers prétextes, teilte Beugnot am 2. April 1813 dem Präfekten Spee mit. Je vous prie de les faire surveiller, et avertir qu'ils s'exposent à se faire envoyer en France [...]. Je dois vous déclarer encore, qu'aucun journal ne doit paraitre [sic] dans votre arrondissement que sous votre censure, et que vous êtes personellement responsable de ce qu'il contient $t^{448}$.

Der zunehmende Verlust an Rechtsgarantien war zum anderen darin spürbar, daß Maßnahmen und Regelungen, die bis zu den Unruhen Ausnahmeerscheinungen zur Bewältigung von Krisensituationen gewesen waren, nach dem Ende der Proteste zur Normalität wurden. Ruhestörer mußten sich nach Beendigung der Unruhen, wie gesagt, weiterhin vor Militärkommissionen verantworten, weil die Regierung sichergehen wollte, daß die Verdächtigten auch tatsächlich bestraft wurden. Ebenso verfestigte sich der Einsatz von Militär bei Auseinandersetzungen zwischen Staat und Gesellschaft. Nach dem bereits erwähnten Zusammensto $ß$ zwischen Gendarmen und Deserteuren in Gevelsberg beispielsweise ordnete Beugnot ohne zu zögern an, französische Truppen in das Arrondissement Hagen zu schicken, pour le purger des brigands qui l'infestent. Ces brigands font nécessairement partie de ces insurrections sur les derrières des armées, dont le baron de Stein a la suprême direction ${ }^{449}$. Der zunehmende Rückgriff auf militärische Hilfe wurde im übrigen dadurch begünstigt, daß die Aufgaben der Hohen Polizei seit Ende Januar 1813 in den Händen des französischen Generals Lemarois, dem höheren Kommandanten der großherzoglich-bergischen Truppen, lagen und damit nicht mehr zum Ressort des Innenministers, sondern zum Zuständigkeitsbereich des französischen Militärs gehörten.

Kulminationspunkt dieser Entwicklungen stellte das Nossener Dekret vom 8. Mai 1813 dar, in dem Napoleon auf den Verdacht eines französischen Spions, während der Unruhen seien Kolonialwaren in das Großherzogtum geschmuggelt worden, anordnete, sämtliche Kolonialwaren, die sich im Großherzogtum befanden, zu beschlagnahmen und nach Köln zum Verkauf zu bringen. Diese Bestimmung galt selbst für jene Waren, für welche die im Großherzogtum Berg gültigen Zolltarife bezahlt worden waren ${ }^{450}$.

447 Wir sind alle, und Beugnot nicht weniger wie wir, mit französischen Policey Spionen umgeben, warnte Nesselrode den Präfekten Romberg in seinem Schreiben vom 3. September 1813, StAM, Nachlaß Giesbert von Romberg A, Nr.8.

448 Schreiben Beugnots an den Präfekten Spee vom 2. April 1813, HStAD, GB, Nr.11100.

449 Beugnot in seinem Schreiben an den General Damas vom 3. Mai 1813, AN, 29 AP 38.

450 Dekret von Nossen vom 8. Mai 1813, Art.1f, AN, 29 AP 34. 
Mit dem Nossener Dekret setzte Napoleon geltendes Recht in bis dahin unbekannter Form außer Kraft. Darüber hinaus mißachtete er in dieser Sache die bergischen Hoheitsrechte vollständig und machte das Großherzogtum zum Spielball der französischen Interessen. Die Ausführung des Dekrets lag nämlich nicht in den Händen der bergischen Behörden, sondern war General Lemarois und dem Weseler Zolldirektor Turc anvertraut, unter deren Leitung mehrere Truppenkolonnen über Wesel und Köln das Großherzogtum durchqueren sollten ${ }^{451}$. Französische Gendarmen und Douanen hatten zwar immer schon im Großherzogtum interveniert. Doch war ihr Einsatz bis dahin nur punktueller Natur gewesen, während es sich jetzt um eine flächendeckende Operation handelte ${ }^{452}$. Einmalig war außerdem die fehlende Transparenz in der Angelegenheit. Das Dekret wurde im Großherzogtum nicht veröffentlicht. Nur der Handelsminister und der Kriegsminister Frankreichs, Jean-Baptiste Henry Collin de Sussy (1750-1826) und Henry-Jacques Clarke (1765-1818), die französischen Douanen, die mit der Ausführung beauftragt waren, sowie Roederer und Beugnot kannten die kaiserliche Verfügung ${ }^{453}$. Einwohner und einheimische Bedienstete, Innenminister Nesselrode eingeschlossen, erfuhren davon erst, als der französische Zoll am 19. Mai 1813 mit der Konfiskation der Waren begann. Daß der kaiserliche Kommissar letztlich im Vorfeld Kenntnis von dem Dekret erhielt, war zunächst auch nicht geplant und ging auf ein Mißverständnis in Paris zurück ${ }^{454}$. Roederer wußte nichts von der strikten Ausklammerung der bergischen Behörden und weihte Beugnot unter dem Siegel der Verschwiegenheit in die Sache ein. Je vous prie, forderte er Beugnot am 14. Mai 1813 auf, als er ihm das Dekret per Estafette überbringen ließ, de prendre les mesures nécessaires pour que l'exécution de ce décret ait lieu promptement, secrètement et a [sic] peu près simultanément sur tous les points du territoire 455 .

Da die Einwohner nichts von der Existenz des Nossener Dekrets wußten, brach unter ihnen größte Verwirrung aus, als die französischen Staatsdiener damit begannen, das Land nach Kolonialwaren zu durchsuchen. Munizipaldiener bezweifelten, daß die von den französischen Douanen vorgenommenen Visitationen rechtmäßig waren, zumal die französischen Beamten oftmals betrunken waren und gewaltsam vorgingen ${ }^{456}$. Einige von ihnen ließen sich in

451 Siehe das Schreiben Roederers an Beugnot vom 17. Mai 1813, ferner das Schreiben Beugnots an Roederer vom 19. Mai 1813, HStAD, GB, Nr. 5590.

452 Im Winter 1811 beispielsweise wurden mobile französische Kolonnen im Großherzogtum eingesetzt, um nach Deserteuren zu suchen. Vgl. den Brief Nesselrodes an Roederer vom 23. November 1811, AN, AF IV* 444.

453 Siehe das Schreiben Roederers an Beugnot vom 17. Mai 1813; ferner das Schreiben Nesselrodes an den General Lemarois vom 22. Mai 1813, HStAD, GB, Nr. 5590.

454 Schreiben des französischen Minister-Staatssekretärs Daru an Roederer vom 8. Mai 1813, AN, 29 AP 34.

455 Schreiben Roederers an Beugnot vom 14. Mai 1813, ibid.

456 Vgl. etwa das Schreiben des Lünener Maires an den Präfekten Romberg vom 21. Mai 1813, HStAD, GB, Nr. 5590. 
den Gemeinden sogar verpflegen ${ }^{457}$. Etliche Unternehmer wehrten sich gegen die Konfiskationen und reklamierten die Rückgabe der beschlagnahmten Güter. Für sie war nicht nachvollziehbar, daß das, was gestern noch gegolten hatte, heute nicht mehr wirksam sein sollte. Der Baumwollfabrikant Johann Gottfried Brügelmann etwa teilte Präfekt Spee am 19. Mai 1813 mit, für die gesamte bei ihm konfiszierte Baumwolle die gesetzlichen Tarife bezahlt und die nötigen Einfuhrlizenzen erhalten zu haben, und bat vor diesem Hintergrund um die Herausgabe der Waren. Er könne unmöglich glauben, beschwerte er sich bei Spee erneut am 14. Juni 1813, que pour prix ce cette confiance illimitée dans la première autorité que Sa Majesté a donnée au Grand-Duché, je dois être condamné à perdre ce qui m'appartient en vertu des loix ${ }^{458}$. Man solle Schuldige nicht mit Unschuldigen verwechseln, fügte Brügelmann hinzu ${ }^{459}$. Damit spielte er auf die Unruhen an und distanzierte sich implizit davon.

Ich kann es mir unmöglich vorstellen, teilte der Düsseldorfer Kaufmann Lindgens Nesselrode am 21.Juni 1813 mit, daß ein Eigenthum, welches ich gleichsam von Sr. Majestät dem Kaiser erworben und folglich durch dieselbe gesichert worden ist, daß ein solches gesetzliches Eigenthum mir auf Befehl Sr. Majestät wieder entrissen werden sollte. Welche Sicherheit bliebe dann im State noch übrig? ${ }^{460}$

Si nous ne pouvons plus compter sur la protection à la quelle notre obéissance aveugle aux lois et la conformité la plus scrupuleuse aux règlements prescrits nous donnent le droit de prétendre, beklagten sich am 21. Juni 1813 auch Gewerbetreibende aus Elberfeld bei Nesselrode, si des opérations faites sous l'autorisation spéciale du représentant de S.M., sanctionnées par sa signature, sont traitées sur le même pied avec des actions de fraude, qui voudra, qui pourra dorenavant faire le commerce? La loyauté la plus stricte, la prudence la plus réservée ne pouvant garantir contre tels évènemens [sic]. Le crédit et la confiance [...] sont sapés et tombent en ruine 461 .

Noch schwerer als der Normenkonflikt wogen die gesellschaftlichen und wirtschaftlichen Implikationen, die mit dem Nossener Dekret verbunden waren. Zahlreiche Fabrikanten mußten ihre Produktion aus Mangel an Rohstoffen einstellen. Davon war insbesondere die Baumwollindustrie betroffen, weil diese bereits unter der vollständigen Anwendung des Tarifs von Trianon litt, die Beugnot auf Befehl Roederers am 4. Mai 1813 angeordnet hatte ${ }^{462}$. Mas-

457 Man beklage sich, teilte Beugnot Roederer am 29. Mai 1813 mit, de la dureté avec laquelle les douaniers français traitaient les magistrats, les citoyens, et même les employés des douanes gd. ducales. M. le Commandant supérieur a donné sur ce point, les ordres les plus sevères; et $M$. Turc a fait autant pour les douaniers en particulier (ibid.). Vgl. ebenso das Schreiben des Lünener Maires an den Präfekten Romberg vom 21.Mai 1813, ibid.

458 Eingabe Brügelmanns an den Präfekten Spee vom 19. Mai 1813, ibid.

459 Ibid.

460 Schreiben Lindgens an den Innenminister vom 21. Juni 1813, ibid.

461 Eingabe des Elberfelder Handels an Nesselrode am 21. Juni 1813, AN, 29 AP 34.

462 HStAD, GB, Nr. 5590; AN, 29 AP 34. 
senentlassungen und Bankrotte waren die Folge. Das Vagantenwesen und die Straßenbettelei nahmen sprunghaft zu. Plündernde Räuberbanden bedrohten zunehmend die innere Sicherheit. Die Kriminalisierung der Einwohner stieg unaufhaltsam. Nach Aussage der einheimischen Behörden verdreifachten sich Diebstahlsdelikte innerhalb eines Monats. Die Zahl der Armen vergrößerte sich drastisch, während die Kommunen, mit rückläufigen Spendenbeiträgen konfrontiert, das Anwachsen der Armut mit kommunalen Mitteln nicht mehr aufzufangen wußten. Wie ist es möglich, stellte Spee Nesselrode gegenüber am 17. Mai 1813 fest, so viel brodlose Familien zu unterhalten, da bei der grösten Einschränkung der Spenden, und bey der grösten Anstrengung der Commissionen in Herbeyschaffung der Fonds diese bisher kaum hinreichten, die Zahl der vorhandenen Armen zu nähren ${ }^{463}$.

Die um ihre Subsistenz gebrachten Arbeiter erwarteten in ihrer Not Hilfe von Kaufleuten und Händlern, die sie für ihr Elend verantwortlich machten $^{464}$. Einige gingen hierbei so weit, mit Hilfe von Brandbriefen - ein noch aus vorfranzösischer Zeit stammendes Artikulationsmittel der Unterschichten, mit dem diese verletzte Gerechtigkeitsvorstellungen oder Rachegedanken zum Ausdruck brachten ${ }^{465}$ - Geld von ihren ehemaligen Arbeitgebern zu erpressen.

Sie werden so gütig seyn, hieß es etwa in einem Brief, der in der Mairie Lüttringhausen gefunden wurde, und legen diesen Abend auf Ihr Feld unten an den Büschen das Eck, da stehet ein Stöckchen mit einem weißen Papier und unter daran einen Beutel in der Erden, da werden Sie uns 100 Rthler Geld einthun; wir sagen und schreiben einhundert Reichsthaler, denn unser sind achte, denn wir müssen es aus großer Noth thun, denn wir haben diesen ganzen Winter keine Arbeit, denn unser sind einge dabey die haben kein Brod zu essen und die haben keine Arbeit und die haben fünf und sechs Kinder und einige dabey, die ausgezogen sind vor die Pacht, ja wir sagen und schreiben, aus groBer Noth thun wir es. Wohere daß Sie das nicht thun, oder uns in Unglück helfen wollen, so wird's Ihnen nicht gut gehen, oder Anstalten dazu machen, daß Sie Wachten ausstellen, das werden wir gewahr, so klug könnt Ihr Euch nicht stellen, dann wird Euch Gott der Herr gewiß strafen, daß Euch alle beyde Scheunen abbrennen, dann werden Sie großen Schaden davon haben ${ }^{466}$.

463 Präfekt Spee in seinem Schreiben an den Innenminister vom 17. Mai 1813, HStAD, GB, Nr.5590; vgl. auch den Brief Nesselrodes an Roederer vom 14. Mai 1813, ibid.

464 Siehe den Brief Nesselrodes an Roederer vom 25. Juni 1813, AN, AF IV 1854.

465 Vgl. die Brandbriefe aus dem 18. Jahrhundert in folgenden Akten: HStAD, JB, Hofrat A, Nr.251, 274. Auch in Frankreich gehörte Brandstiftung zur Protestkultur der Unterschichten. Siehe Jean-Pierre Jessenne, Communautés, communes rurales et pouvoirs dans l'État napoléonien, in: PetrTeau, Voies nouvelles, S. 161-180, hier S. 169f.

466 Anonymer und undatierter Brandbrief an die Gebrüder Graber in der Mairie Lüttringhausen (Frühjahr 1813), AN, AF IV 1854. Brandbriefe wurden auch in den Mairien Duisburg, Gladbach und Wermelskirchen gefunden. In Remscheid kam es Ende Mai 1813 sogar tatsächlich zu einer Brandstiftung. Vgl. den Brandbrief vom 2. April 1807, HStAD, JB, 
Die Düsseldorfer Minister verfolgten die Entwicklungen im Großherzogtum mit größter Sorge. Sie befürchteten, daß die Zunahme von Arbeitslosigkeit, Emigration, Armut und Delinquenz das Vertrauen der Bevölkerung in den Staat und seine Institutionen gänzlich erschüttern und einen erneuten Aufruhr heraufbeschwören könnten. Zudem hielten sie die Vorwürfe, die zum Erlaß des Dekrets geführt hatten, für ungerechtfertigt. Sie waren davon überzeugt, daß während der Unruhen keine Waren unrechtmäßig in das Großherzogtum gelangt waren. Deshalb setzten sie sich auch nachdrücklich dafür ein, den Inhalt des Dekrets nur teilweise auszuführen ${ }^{467}$. Der Innenminister entsandte zu diesem Zweck sogar eine zweiköpfige Deputation zum Kaiser nach Dresden. Sie sollte erreichen, daß Napoleon einen Bericht in dieser Angelegenheit erstellen ließ und den Verkauf der konfiszierten Waren bis dahin aufschob ${ }^{468}$. Die Bemühungen der beiden Ressortleiter, Erleichterungen für Wirtschaft und Handel zu erwirken, scheiterten jedoch. Napoleon hielt an seinem ursprünglichen Entschluß fest, die konfiszierten Waren nach Köln transportieren und dort verkaufen zu lassen. Nur die Baumwolle, für welche die französischen Tarife entrichtet worden waren, durfte im Besitz der Fabrikanten bleiben ${ }^{469}$.

Erlaß und Vollzug des Nossener Dekrets machen deutlich, daß das Großherzogtum Berg für Napoleon im Frühjahr 1813 keine Relevanz mehr besaß und vollständig zum Spielball seiner machtpolitischen Interessen geworden war. Nesselrode und Beugnot verteidigten zwar die Belange der Einwohner und versuchten ein Minimum an Rechtsschutz zu erhalten. Doch konnten sie sich gegenüber Napoleon nicht durchsetzen. Diese Entwicklungen hatten zum einen zur Folge, daß die französische Herrschaft im Mai 1813 für die Bewohner des Großherzogtums jegliche Berechenbarkeit verloren hatte und von den Ansprüchen moderner Rechtsstaatlichkeit noch weit entfernt war. Zum anderen verursachten sie Akzeptanz- und Vertrauensverluste der französischen Herrschaft in der Einwohnerschaft und führten dazu, daß das Ansehen des Staates beschädigt blieb. Der Anstoß für den Zusammenbruch des Großherzogtums im Winter $1813 \mathrm{kam}$ zwar letztlich von außen. Aber das Ende der französischen Herrschaft kündigte sich bereits durch diese zunehmende Destabilisierung im Landesinnern an.

Hofrat A, Nr.251, ferner das Schreiben des Prokurators des Düsseldorfer Erstinstanzgerichts an Nesselrode vom 22. Juni 1813, AN, AF IV 1854.

467 Vgl. das Schreiben Nesselrodes an Roederer vom 21. Mai 1813, AN, 29 AP 34, das Schreiben Nesselrodes an Roederer vom 5.Juni 1813, HStAD, GB, Nr. 5590, das Schreiben Beugnots an Roederer vom 11.Juni 1813, AN, AF IV 1854, den Avis du Commissaire Impérial sur les Réclamations des Chambres de commerce et des Négocians du Grand Duché de Berg, contre le Décret rendu à Nossen le 8 Mai vom 23. Juni 1813, AN, 29 AP 34, sowie das Schreiben Beugnots an Roederer vom 24. Juni 1813, HStAD, GB, Nr. 5590.

468 Undatierte Depesche Nesselrodes an Napoleon (Frühjahr 1813); Brief Roederers an Beugnot vom 28. Juni 1813, AN, 29 AP 34.

469 Anweisung Napoleons vom 16.Juni 1813, HStAD, GB, Nr. 5590. 
